\title{
Point-Based Computer Graphics
}

\author{
Eurographics 2002 Tutorial T6
}

\author{
Organizers \\ Markus Gross \\ ETH Zürich \\ Hanspeter Pfister \\ MERL, Cambridge
}

\section{Presenters}

Marc Alexa

TU Darmstadt

Markus Gross

ETH Zürich

Mark Pauly

ETH Zürich

Hanspeter Pfister

MERL, Cambridge

Marc Stamminger

Bauhaus-Universität Weimar

Matthias Zwicker

ETH Zürich 


\section{Contents}

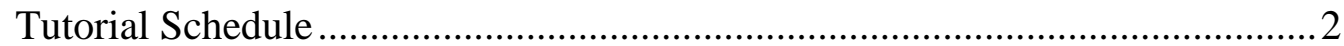

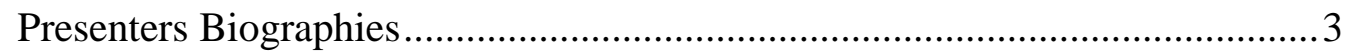

Presenters Contact Information .................................................................... 4

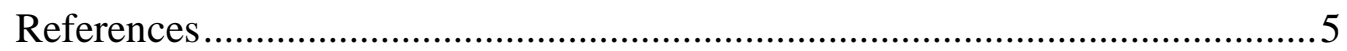

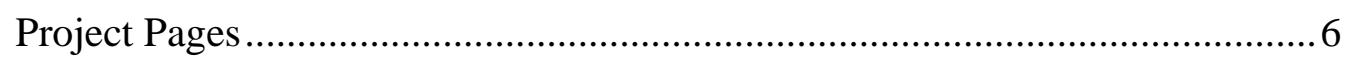

\section{Tutorial Schedule}

8:30-8:45 Introduction (M. Gross)

8:45-9:45 Point Rendering (M. Zwicker)

9:45-10:00 Acquisition of Point-Sampled Geometry and Appearance I

(H. Pfister)

10:00-10:30 Coffee Break

10:30-11:15 Acquisition of Point-Sampled Geometry and Appearance II (H. Pfister)

11:15-12:00 Dynamic Point Sampling (M. Stamminger)

12:00-14:00 Lunch

14:00-15:00 Point-Based Surface Representations (M. Alexa)

15:00-15:30 Spectral Processing of Point-Sampled Geometry (M. Gross)

15:30-16:00 Coffee Break

16:00-16:30 Efficient Simplification of Point-Sampled Geometry (M. Pauly)

16:30-17:15 Pointshop3D: An Interactive System for Point-Based Surface Editing (M. Pauly)

17:15-17:30 Discussion (all) 


\section{Presenters Biographies}

Dr. Markus Gross is a professor of computer science and the director of the computer graphics laboratory of the Swiss Federal Institute of Technology (ETH) in Zürich. He received a degree in electrical and computer engineering and a Ph.D. on computer graphics and image analysis, both from the University of Saarbrucken, Germany. From 1990 to 1994 Dr. Gross was with the Computer Graphics Center in Darmstadt, where he established and directed the Visual Computing Group. His research interests include physics-based modeling, point based methods and multiresolution analysis. He has widely published and lectured on computer graphics and scientific visualization and he authored the book "Visual Computing", Springer, 1994. Dr. Gross has taught courses at major graphics conferences including SIGGRAPH, IEEE Visualization, and Eurographics. He is associate editor of the IEEE Computer Graphics and Applications and has served as a member of international program committees of major graphics conferences. Dr. Gross was a papers co-chair of the IEEE Visualization '99 and Eurographics 2000 conferences.

Dr. Hanspeter Pfister is Associate Director and Senior Research Scientist at MERL - Mitsubishi Electric Research Laboratories - in Cambridge, MA. He is the chief architect of VolumePro, Mitsubishi Electric's real-time volume rendering hardware for PCs. His research interests include computer graphics, scientific visualization, and computer architecture. His work spans a range of topics, including point-based rendering and modeling, 3D scanning, and computer graphics hardware. Hanspeter Pfister received his Ph.D. in Computer Science in 1996 from the State University of New York at Stony Brook. He received his M.S. in Electrical Engineering from the Swiss Federal Institute of Technology (ETH) Zurich, Switzerland, in 1991. He is Associate Editor of the IEEE Transactions on Visualization and Computer Graphics (TVCG), member of the Executive Committee of the IEEE Technical Committee on Graphics and Visualization (TCVG), and member of the ACM, ACM SIGGRAPH, IEEE, the IEEE Computer Society, and the Eurographics Association.

Mark Pauly is currently a PhD student at the Computer Graphics Lab at ETH Zurich, Switzerland. He is working on point-based surface representations for 3D digital geometry processing, focusing on spectral methods for surface filtering and resampling. Further research activities are directed towards multiresolution modeling, geometry compression and texture synthesis of point-sampled objects.

Dr. Marc Stamminger received his $\mathrm{PhD}$ in computer graphics in 1999 from the University of Erlangen, Germany, for his work about finite element methods for global illumination computations. After that he worked at the Max-Planck-Institut for Computer Science (MPII) in Saarbrücken, Germany, where he headed the global illumination group. As a PostDoc in Sophia-Antipolis in France he worked on the interactive rendering and modeling of natural environments. Since 2001 he is an assistant professor at the Bauhaus-University in Weimar. His current research interests are point-based methods for complex, dynamic scenes, and interactive global illumination methods.

Matthias Zwicker is in his last year of the $\mathrm{PhD}$ program at the Computer Graphics Lab at ETH Zurich, Switzerland. He has developed rendering algorithms and data 
structures for point-based surface representations, which he presented in the papers sessions of SIGGRAPH 2000 and 2001. He has also extended this work towards high quality volume rendering. Other research interests concern compression of point-based data structures, acquisition of real world objects, and texturing of point-sampled surfaces.

Dr. Marc Alexa leads the project group "3d Graphics Computing" within the Interactive Graphics System Group, TU Darmstadt. He received his PhD and MS degrees in Computer Science with honors from TU Darmstadt. His research interests include shape modeling, transformation and animation as well as conversational user interfaces and information visualization.

\title{
Presenters Contact Information
}

\author{
Dr. Markus Gross \\ Professor \\ CH 8092 Zürich \\ Switzerland \\ Phone: +41 16327114 \\ FAX: +41 16321596 \\ grossm@inf.ethz.ch \\ http://graphics.ethz.ch
}

Department of Computer Science

Swiss Federal Institute of Technology (ETH)

\section{Dr. Hanspeter Pfister}

Associate Director

MERL - A Mitsubishi Electric Research Lab

201 Broadway

Cambridge, MA 02139

USA

Phone: (617) 621-7566

Fax: (617) 621-7550

pfister@merl.com

http://www.merl.com/people/pfister/

\section{Matthias Zwicker}

Department of Computer Science

Swiss Federal Institute of Technology (ETH)

CH 8092 Zürich

Switzerland

Phone: +41 16327437

FAX: +41 16321596

zwicker@inf.ethz.ch

http://graphics.ethz.ch

\section{Mark Pauly}

Department of Computer Science

Swiss Federal Institute of Technology (ETH)

CH 8092 Zürich 
Switzerland

Phone: +41 16320906

FAX: +4116321596

pauly@inf.ethz.ch

http://graphics.ethz.ch

Dr. Marc Stamminger

Bauhaus-Universität Weimar

Bauhausstr. 11

99423 Weimar

Germany

Phone: +49 3643583733

FAX: +49 3643583709

Marc.Stamminger@medien.uni-weimar.de

Dr. Marc Alexa

Interactive Graphics Systems Group

Technische Universität Darmstadt

Fraunhoferstr. 5

64283 Darmstadt

Germany

Phone: +496151 155674

FAX: +496151 155669

alexa@gris.informatik.tu-darmstadt.de

http://www.igd.fhg.de/ alexa

\section{References}

M. Alexa, J. Behr, D. Cohen-Or, S. Fleishman, D. Levin, C. Silva.

Point set surfaces. Proceedings of IEEE Visualization 2001, p. 21-28, San Diego, CA, October 2001.

O. Deussen, C. Colditz, M. Stamminger, G. Drettakis, Interactive visualization of complex plant ecosystems. Proceedings of IEEE Visualization 2002, to appear, Boston, MA, October 2002.

W. Matusik, H. Pfister, P. Beardsley, A. Ngan, R. Ziegler, L. McMillan, Imagebased 3D photography using opacity hulls. Proceedings of SIGGRAPH 2002, to appear, San Antonio, TX, July 2002.

W. Matusik, H. Pfister, A. Ngan, R. Ziegler, L. McMillan, Acquisition and rendering of transparent and refractive objects. Thirteenth Eurographics Workshop on Rendering, to appear, Pisa, Italy, June 2002.

M. Pauly, M. Gross, Spectral processing of point-sampled geometry. Proceedings of SIGGRAPH 2001, p. 379-386, Los Angeles, CA, August 2001.

M. Pauly, M. Gross, Efficient Simplification of Point-Sampled Surfaces. IEEE Proceedings of Visualization 2002, to appear, Boston, MA, October 2002. 
H. Pfister, M. Zwicker, J. van Baar, M. Gross, Surfels - surface elements as rendering primitives. Proceedings of SIGGRAPH 2000, p. 335-342, New Orleans, LS, July 2000.

M. Stamminger, G. Drettakis, Interactive sampling and rendering for complex and procedural geometry, Rendering Techniques 2001, Proceedings of the Eurographics Workshop on Rendering 2001, June 2001.

L. Ren, H. Pfister, M. Zwicker, Object space EWA splatting: a hardware accelerated approach to high quality point rendering. Proceedings of the Eurographics 2002, to appear, Saarbrücken, Germany, September 2002.

M. Zwicker, H. Pfister, J. van Baar, M. Gross, EWA volume splatting.

Proceedings of IEEE Visualization 2001, p. 29-36, San Diego, CA, October 2001.

M. Zwicker, H. Pfister, J. van Baar, M. Gross, Surface splatting.

Proceedings of SIGGRAPH 2001, p. 371-378, Los Angeles, CA, August 2001.

M. Zwicker, H. Pfister, J. van Baar, M. Gross, EWA splatting. IEEE Transactions on Visualization and Computer Graphics, to appear.

M. Zwicker, M. Pauly, O. Knoll, M. Gross, Pointshop 3D: an interactive system for point-based surface editing. Proceedings of SIGGRAPH 2002, to appear, San Antonio, TX, July 2002

\section{Project Pages}

- Rendering http://graphics.ethz.ch/surfels

- Acquisition http://www.merl.com/projects/3Dimages/

- Dynamic sampling http://www-sop.inria.fr/reves/personnel/Marc.Stamminger/pbr.html

- Processing, sampling and filtering http://graphics.ethz.ch/points

- Pointshop3D http://www.pointshop3d.com 

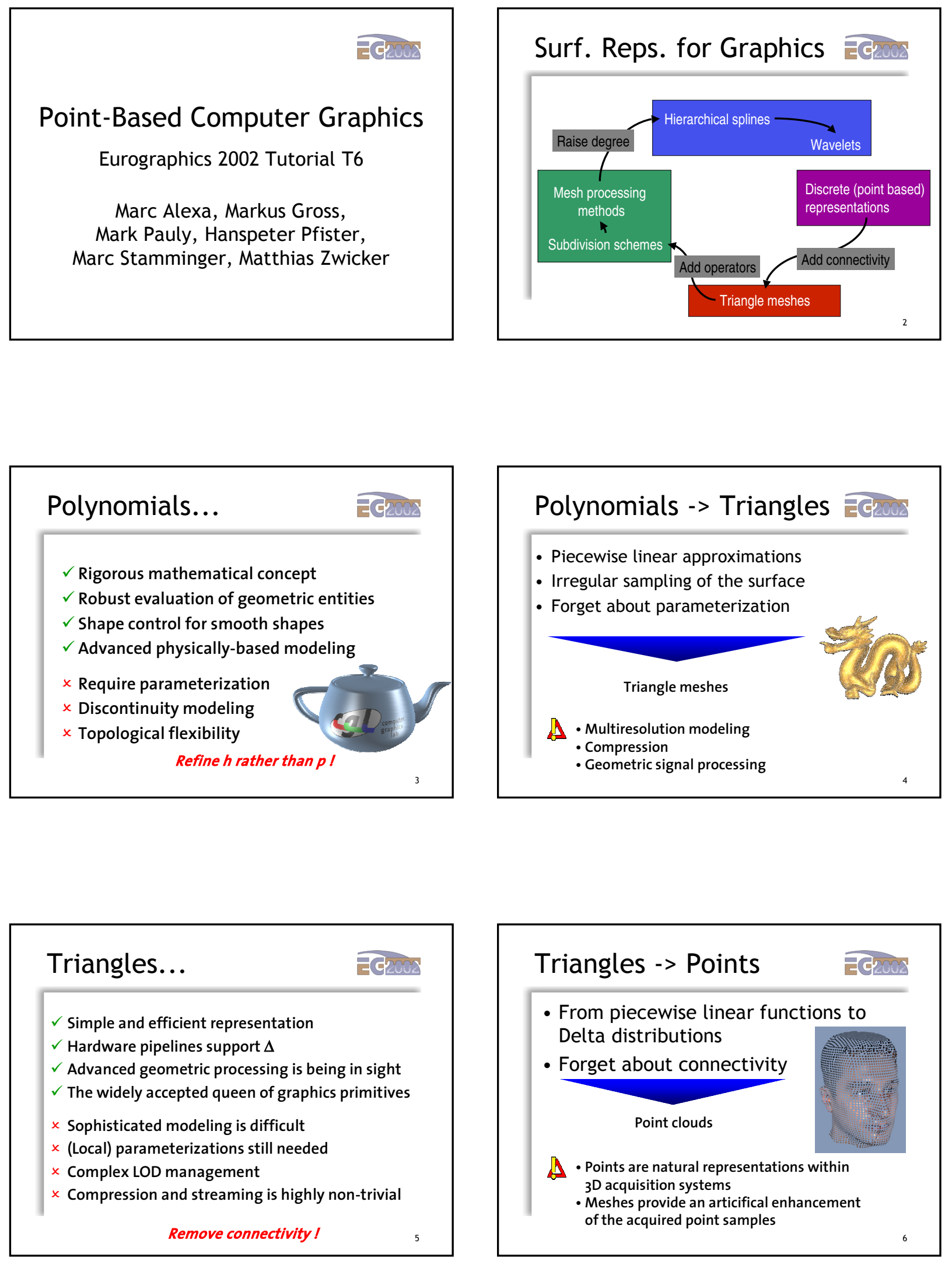


\section{History of Points in Graphics}

- Particle systems [Reeves 1983]

- Points as a display primitive [Whitted, Levoy 1985]

- Oriented particles [Szeliski, Tonnesen 1992]

- Particles and implicit surfaces [Witkin, Heckbert 1994]

- Digital Michelangelo [Levoy et al. 2000]

- Image based visual hulls [Matusik 2000]

- Surfels [Pfister et al. 2000]

- QSplat [Rusinkiewicz, Levoy 2000]

- Point set surfaces [Alexa et al. 2001]

- Radial basis functions [Carr et al. 2001]

- Surface splatting [Zwicker et al. 2001]

- Randomized z-buffer [Wand et al. 2001]

- Sampling [Stamminger, Drettakis 2001]

- Opacity hulls [Matusik et al. 2002]

- Pointshop3D [Zwicker, Pauly, Knoll, Gross 2002]...?
The Purpose of our Course is ...

I) ...to introduce points as a versatile and powerful graphics primitive

II) ...to present state of the art concepts for acquisition, representation, processing and rendering of point sampled geometry

III) ...to stimulate YOU to help us to further develop Point Based Graphics

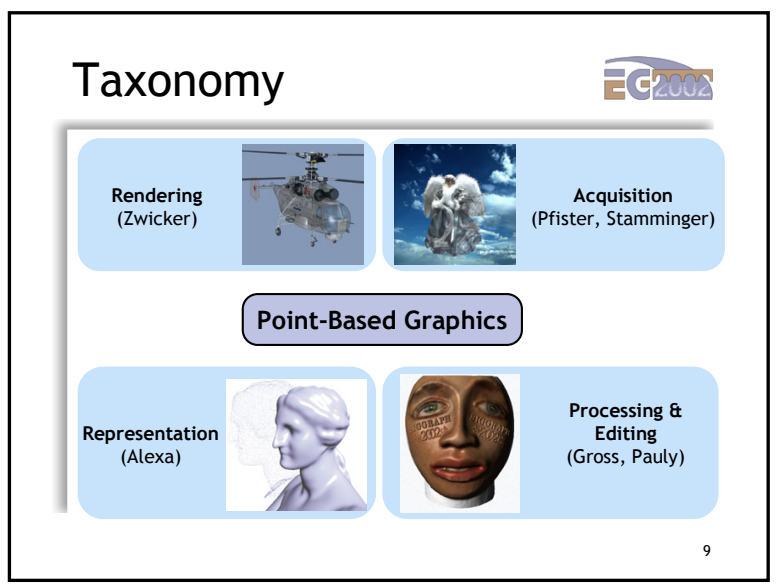

\section{Afternoon Schedule}

14:00-15:00 Point-Based Surface Representations ( $M$. Alexa)

15:00-15:30 Spectral Processing of Point-Sampled Geometry (M. Gross)

15:30-16:00 Coffee Break

16:00-16:30 Efficient Simplification of Point-Sampled Geometry (M. Pauly)

16:30-17:15 Pointshop3D: An Interactive System for PointBased Surface Editing (M. Pauly)

17:15-17:30 Discussion (all)

\section{Morning Schedule}

8:30-8:45 Introduction (M. Gross)

8:45-9:45 Point Rendering (M. Zwicker)

9:45-10:00

Acquisition of Point-Sampled Geometry and Appearance I (H. Pfister)

$10: 00-10: 30$

Coffee Break

10:30-11:15 Acquisition of Point-Sampled Geometry and Appearance II (H. Pfister)

11:15-12:00 Dynamic Point Sampling (M. Stamminger) 


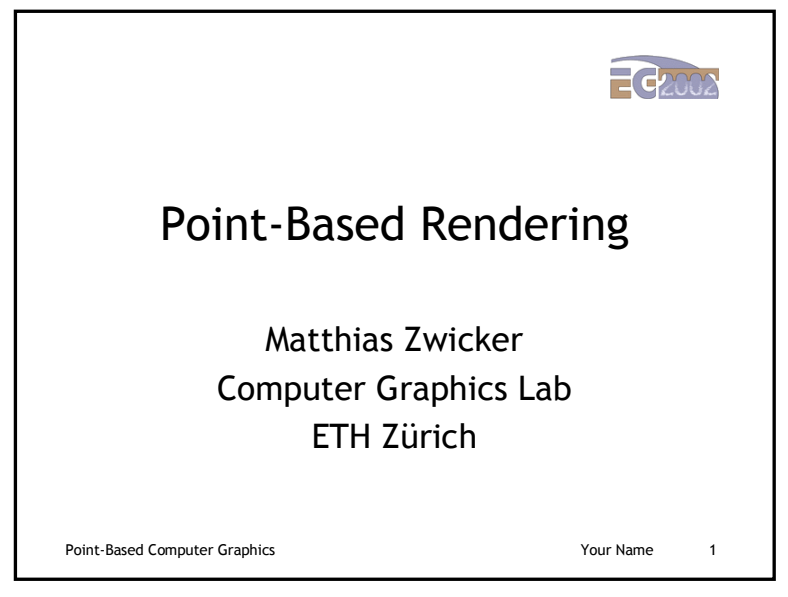

\begin{tabular}{|l|}
\hline Point-Based Rendering \\
\hline - Introduction and motivation \\
- Surface elements \\
- Rendering \\
- Antialiasing \\
- Hardware Acceleration \\
- Conclusions \\
\\
\end{tabular}

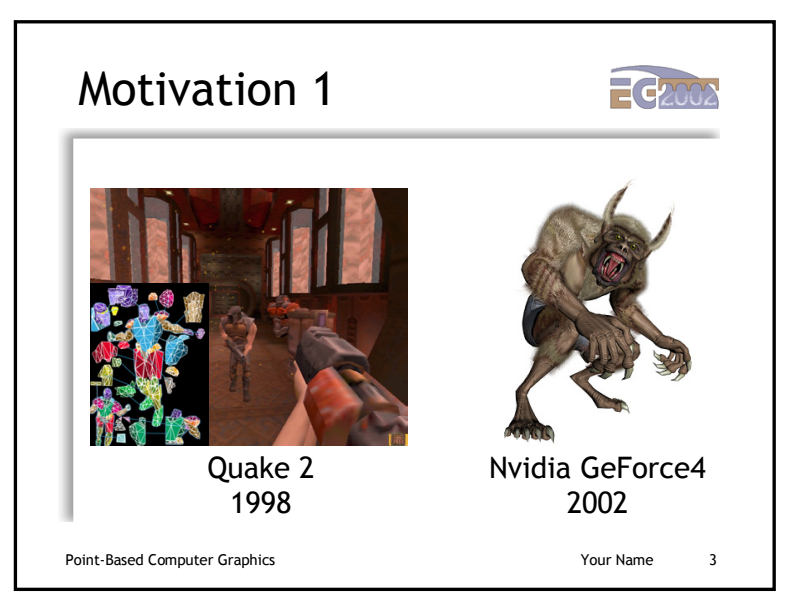

Motivation 1

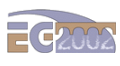

- Performance of 3D hardware has exploded (e.g., GeForce4: 136 million vertices per second)

- Projected triangles are very small (i.e., cover only a few pixels)

- Overhead for triangle setup increases (initialization of texture filtering, rasterization)

A simpler, more efficient rendering primitive than triangles?
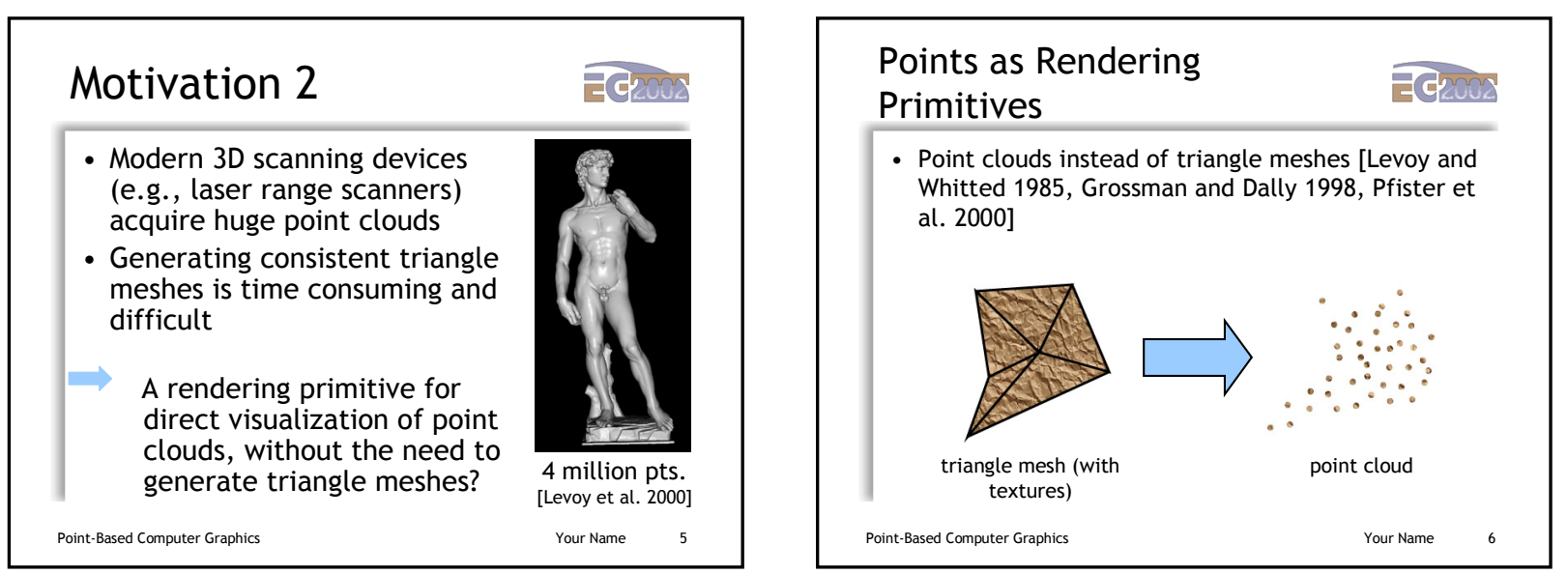

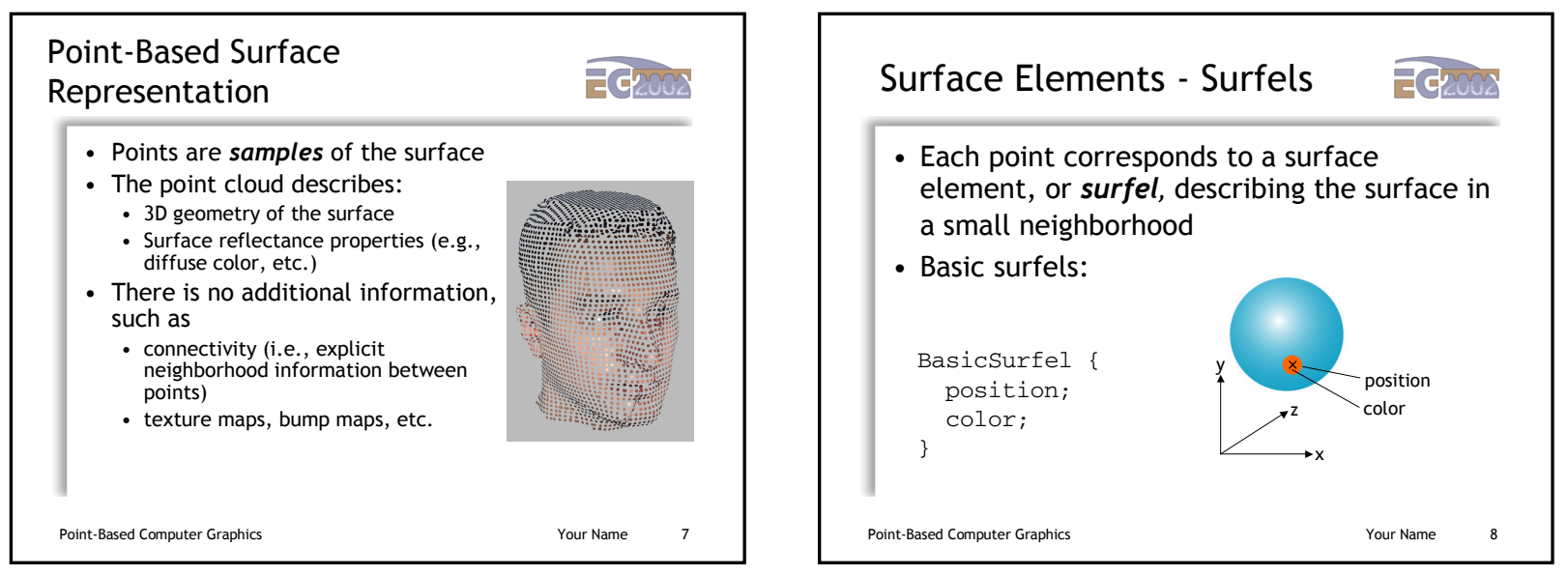

\section{Surfels}

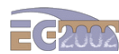

- How to represent the surface between the points?

holes between the points

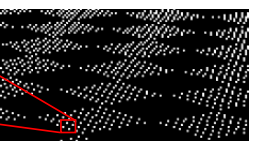

- Surfels need to interpolate the surface between the points

- A certain surface area is associated with each surfel

\section{Surfels}

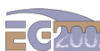

- Surfels store essential information for rendering

- Surfels are primarily designed as a point rendering primitive

- They do not provide a mathematically smooth surface definition (see [Alexa 2001], point set surfaces)

\section{Model Acquisition}

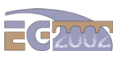

- 3D scanning of physical objects

- See Pfister, acquisition

- Direct rendering of acquired point clouds

- No mesh reconstruction necessary
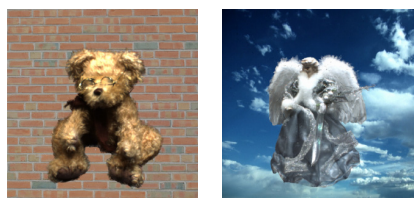

[Matusik et al. 2002] 

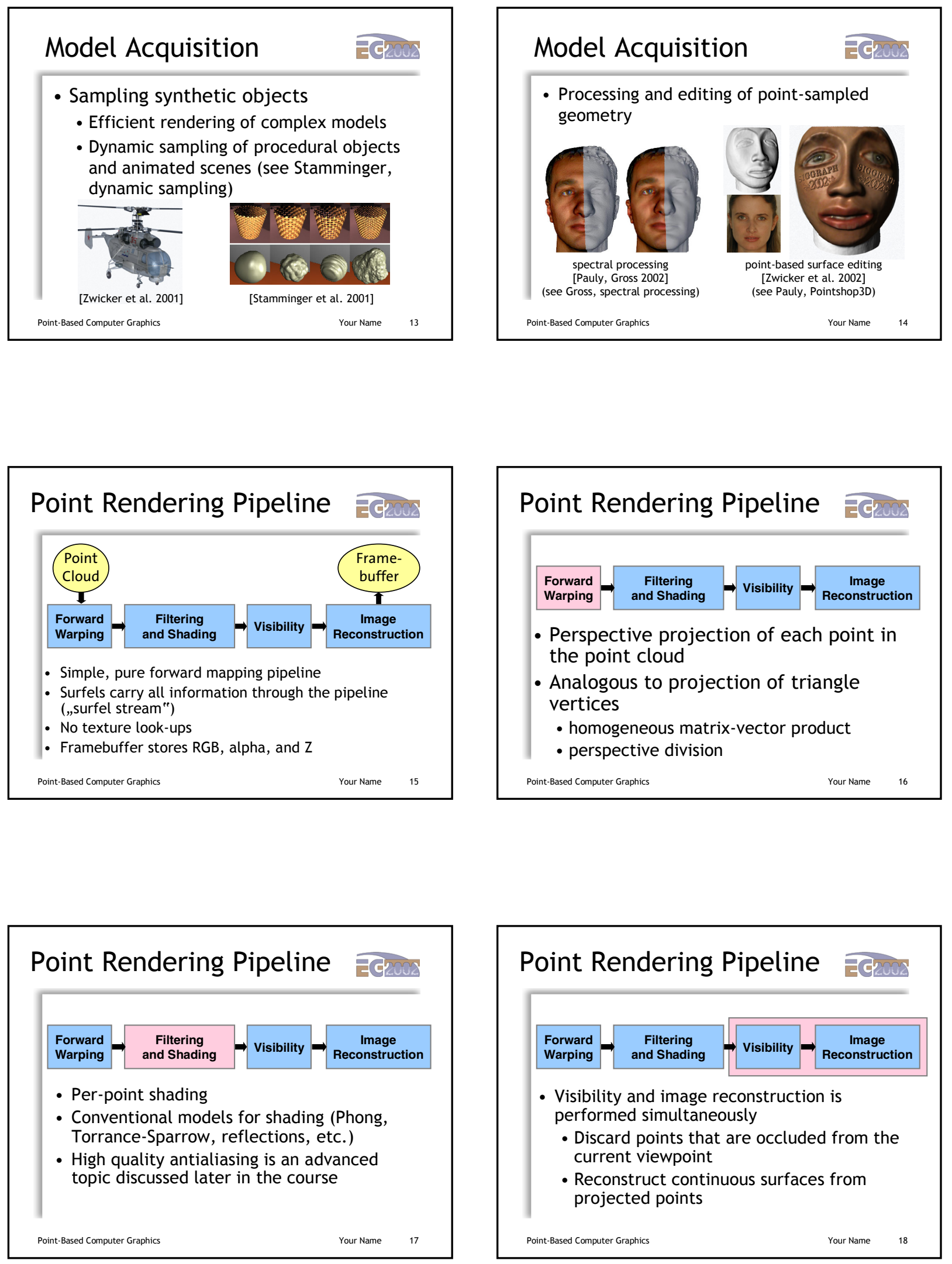

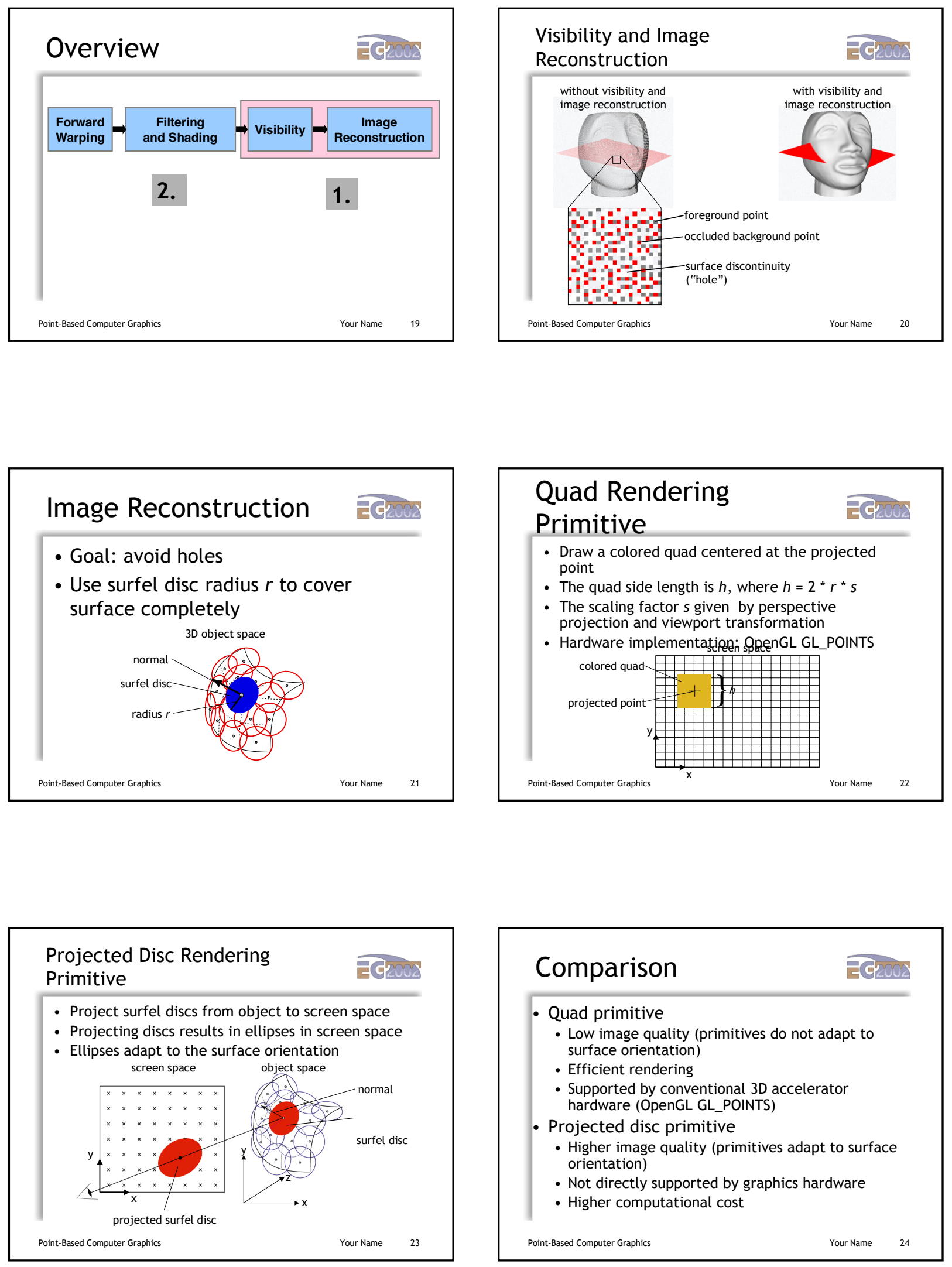

\begin{tabular}{|l|}
\hline Comparison \\
- Quad primitive \\
- Low image quality (primitives do not adapt to \\
surface orientation) \\
- Efficient rendering \\
- Supported by conventional 3D accelerator \\
hardware (OpenGL GL_POINTS) \\
Projected disc primitive \\
- Higher image quality (primitives adapt to surface \\
orientation) \\
- Not directly supported by graphics hardware \\
Poigher computational cost \\
\end{tabular}



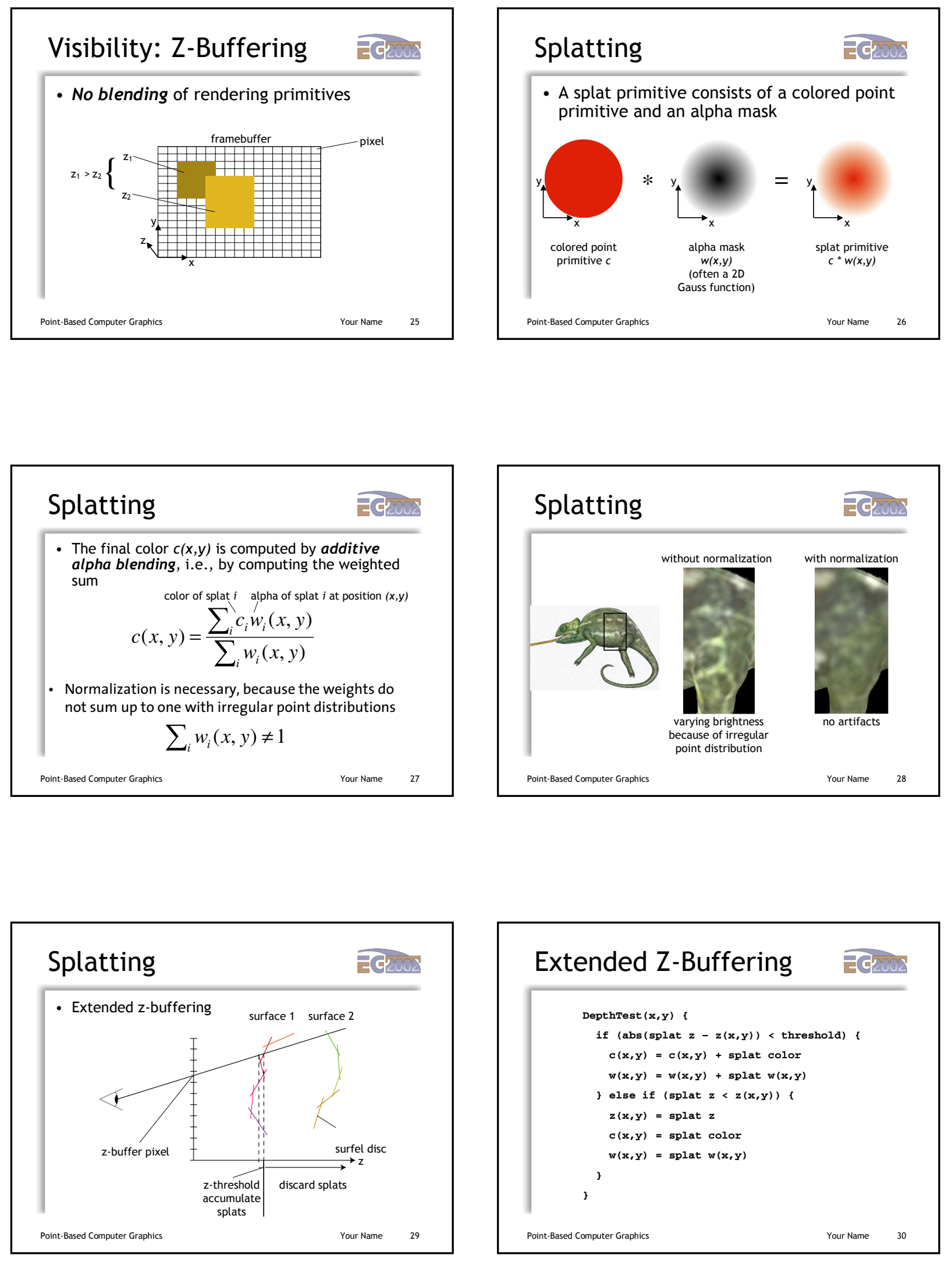


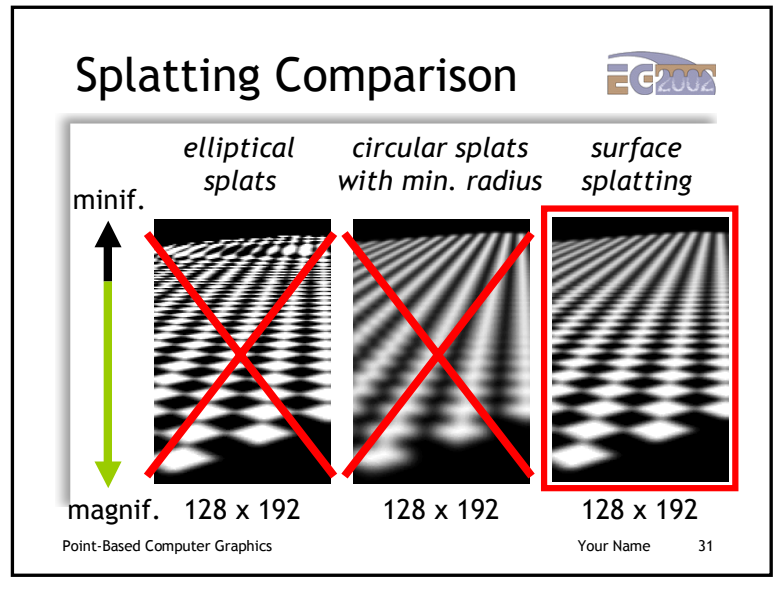

Aliasing in Computer

Graphics

- Aliasing = Sampling of continuous functions below the Nyquist frequency

- To avoid aliasing, sampling rate must be twice as high as the maximum frequency in the signal

- Aliasing effects:

- Loss of detail

- Moire patterns, jagged edges

- Disintegration of objects or patterns

- Aliasing in Computer Graphics

- Texture Mapping

- Scan conversion of geometry

\section{High Quality Splatting}

- High quality splatting requires careful analysis of aliasing issues

- Review of signal processing theory

- Application to point rendering

- Surface splatting [Zwicker et al. 2001]
Aliasing in Computer Graphics

- Aliasing: high frequencies in the input signal appear as low frequencies in the reconstructed signal

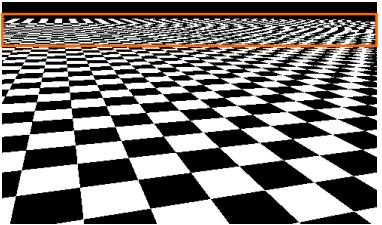

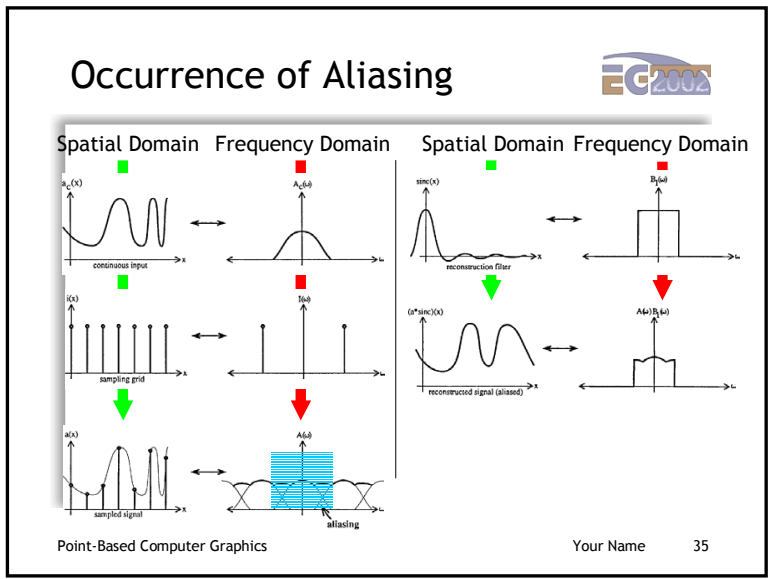

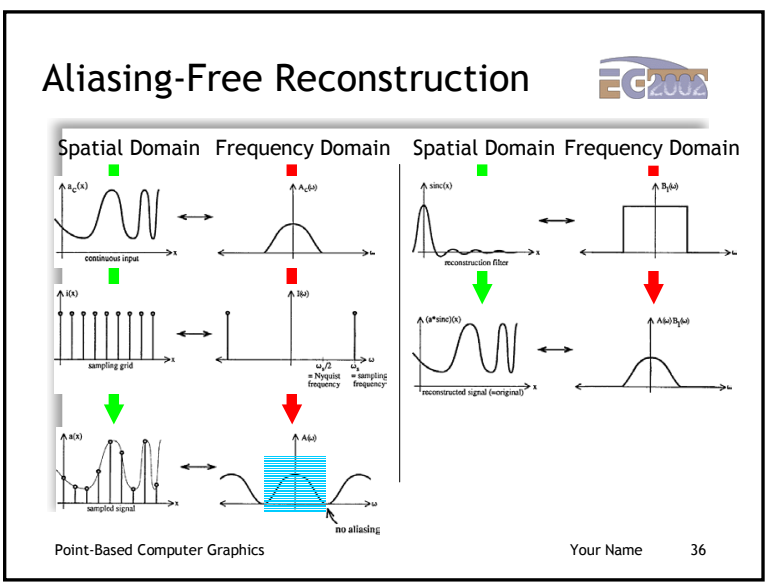



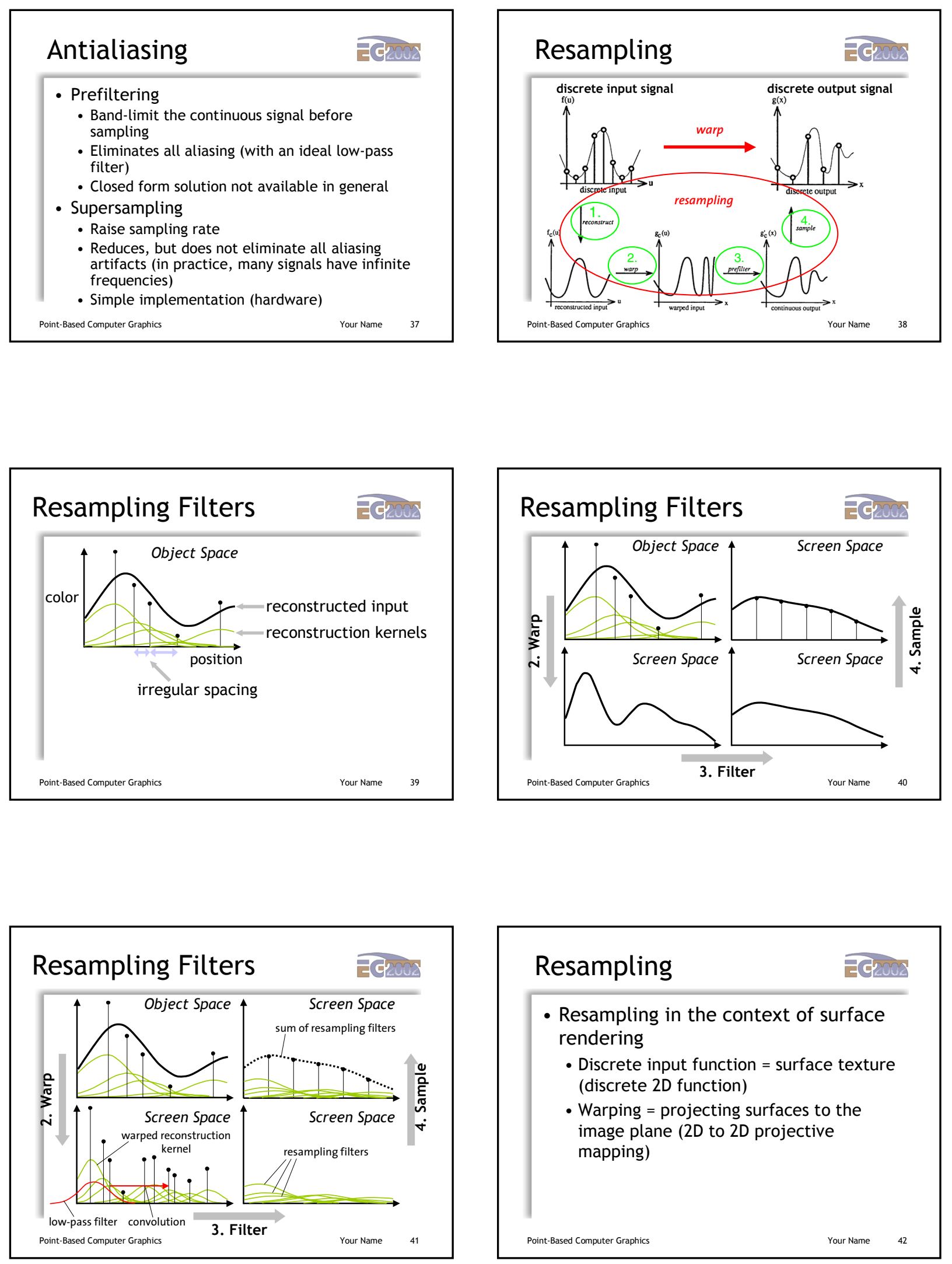

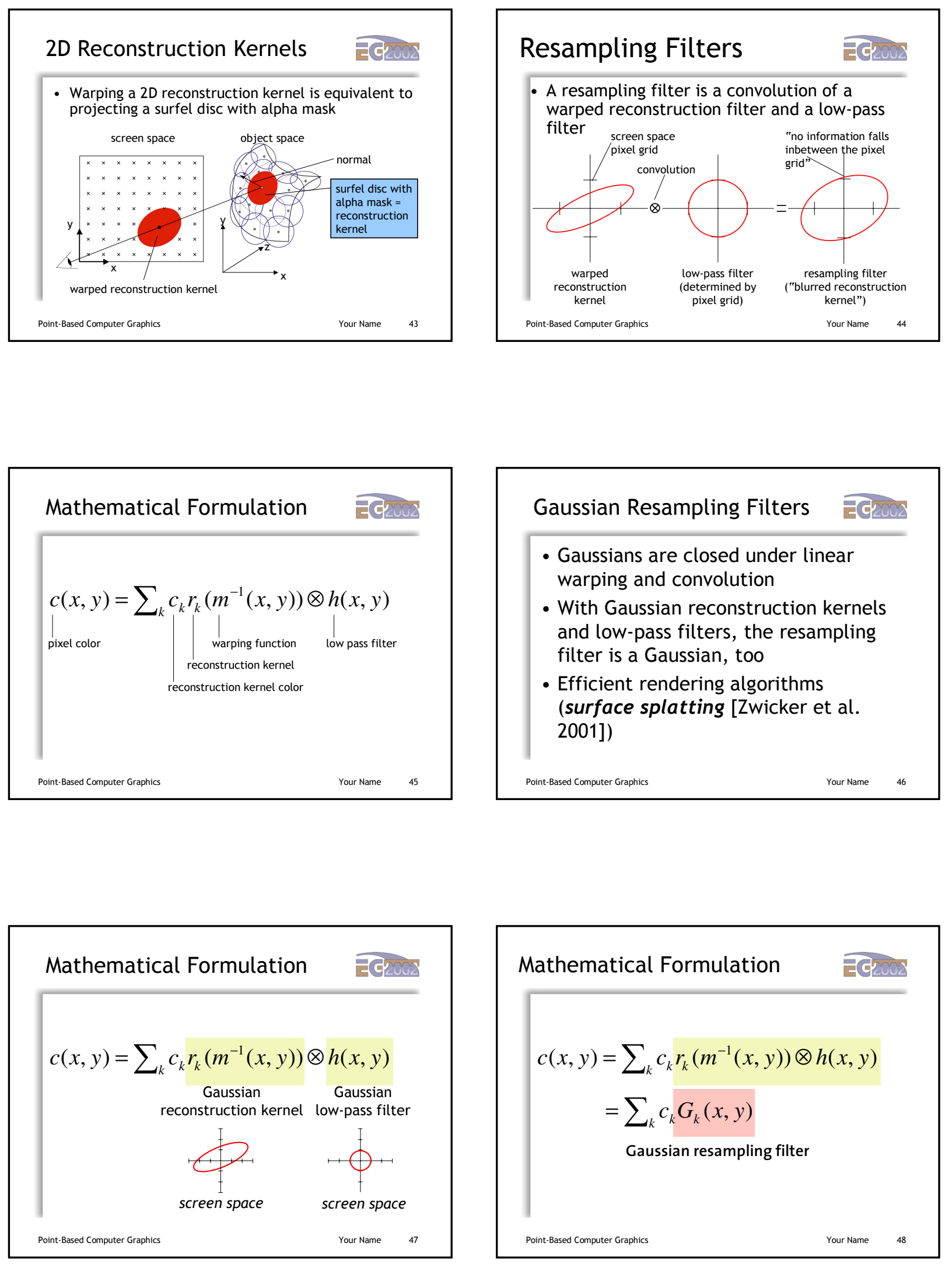


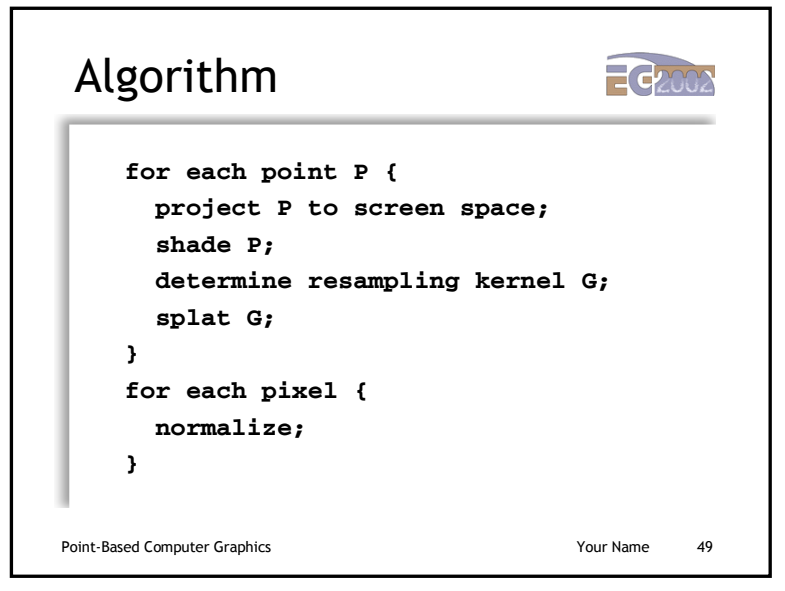

Hardware Implementation

\section{तec0000}

- Based on the object space formulation of EWA filtering

- Implemented using textured triangles

- All calculations are performed in the programmable hardware (extensive use of vertex shaders)

- Presented at EG 2002 ([Ren et al. 2002])

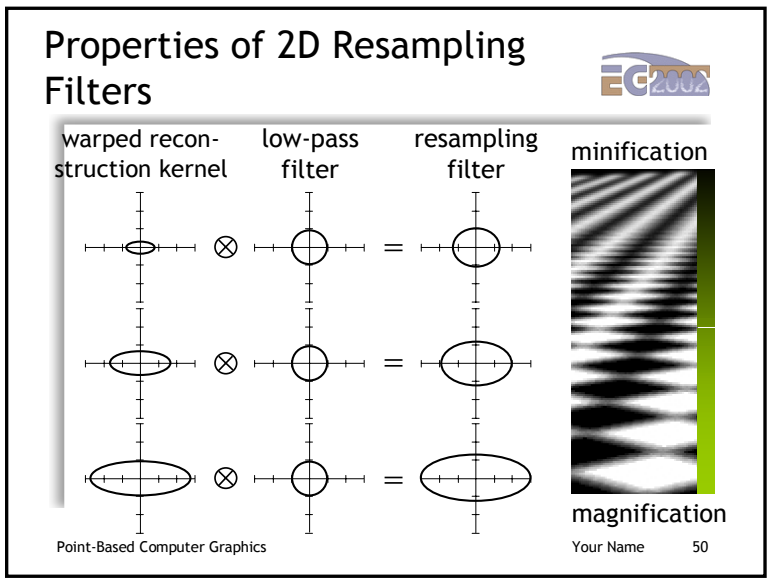

Surface Splatting

Performance

- Software implementation

- 500000 splats/sec on 866 MHz PIII

- 1000000 splats/sec on $2 \mathrm{GHz}$ P4

- Hardware implementation [Ren et al. 2002]

- Uses texture mapping and vertex shaders

- 3000000 splats/sec on GeForce4 Ti 4400

Point-Based Computer Graphics

Your Name 52

\section{Conclusions}

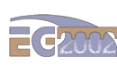

- Points are an efficient rendering primitive for highly complex surfaces

- Points allow the direct visualization of real world data acquired with 3D scanning devices

- High performance, low quality point rendering is supported by $3 \mathrm{D}$ hardware (tens of millions points per second)

- High quality point rendering with anisotropic texture filtering is available

- 3 million points per second with hardware support

- 1 million points per second in software

- Antialiasing technique has been extended to volume rendering

\section{Applications}

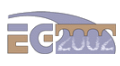

- Direct visualization of point clouds

- Real-time 3D reconstruction and rendering for virtual reality applications

- Hybrid point and polygon rendering systems

- Rendering animated scenes

- Interactive display of huge meshes

- On the fly sampling and rendering of procedural objects 


\begin{tabular}{|l|}
\hline Future Work \\
- Dedicated rendering hardware \\
- Efficient approximations of exact EWA \\
splatting \\
- Rendering architecture for on the fly \\
sampling and rendering \\
\end{tabular}

\section{References}

[Levoy and Whitted 1985] The use of points as a display primitive, technical report, University of North Carolina at Chapel Hill, 1985 [Heckbert 1986] Fundamentals of texture mapping and image warping, Master's Thesis, 1986

[Grossman and Dally 1998] Point sample rendering, Eurographics

workshop on rendering, 1998

[Levoy et al. 2000] The digital Michelangelo project, SIGGRAPH 2000

[Rusinkiewicz et al. 2000] Qsplat, SIGGRAPH 2000

[Pfister et al. 2000] Surfels: Surface elements as rendering primitives,

SIGGRAPH 2000

[Zwicker et al. 2001] Surface splatting, SIGGRAPH 2001

[Zwicker et al. 2002] EWA Splatting, to appear, IEEE TVCG 2002

[Ren et al. 2002] Object space EWA splatting: A hardware accelerated

approach to high quality point rendering, Eurographics 2002 

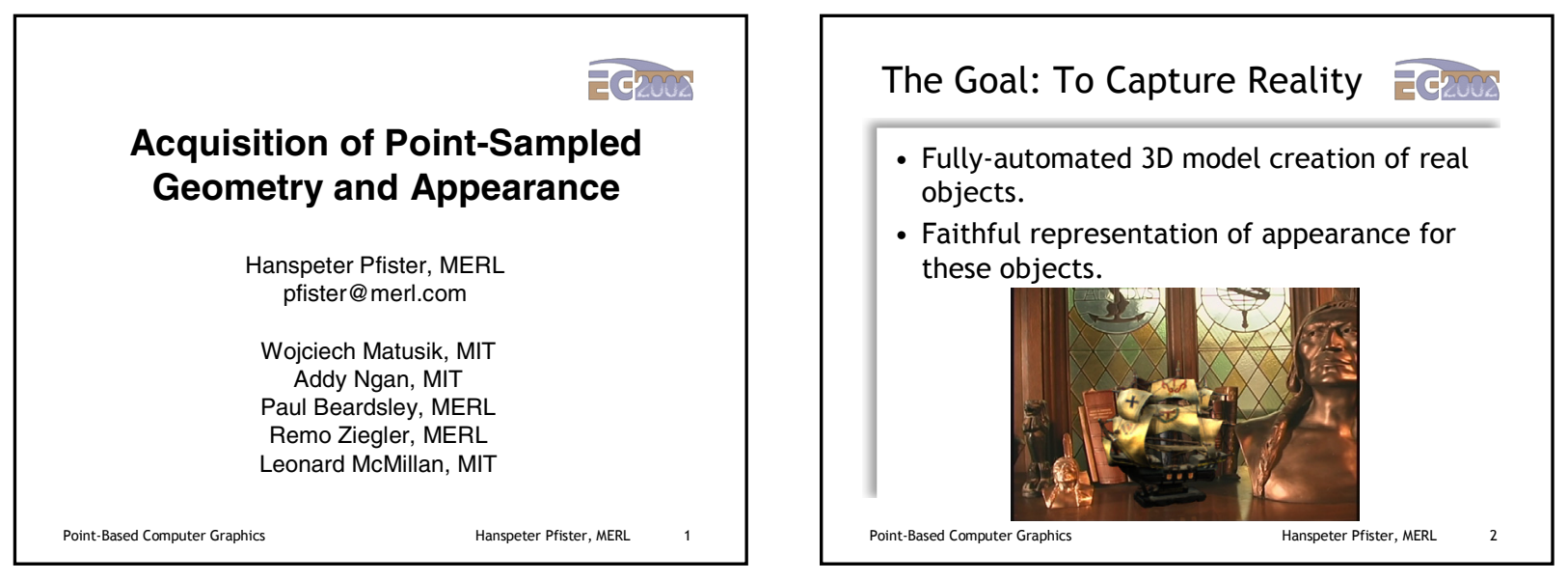

\section{Image-Based 3D Photography}

- An image-based 3D scanning system.

- Handles fuzzy, refractive, transparent objects.

- Robust, automatic

- Point-sampled geometry based on the visual hull.

- Objects can be rendered in novel environments.

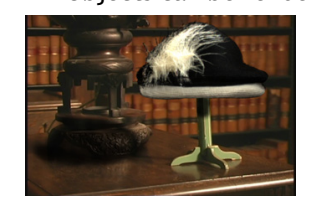

Point-Based Computer Graphics

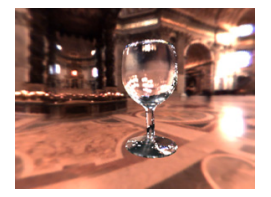

Hanspeter Pfister, MERL

\section{Previous Work}

\section{Fenoor}

- Active and passive 3D scanners

- Work best for diffuse materials.

- Fuzzy, transparent, and refractive objects are difficult.

- BRDF estimation, inverse rendering

- Image based modeling and rendering

- Reflectance fields [Debevec et al. 00]

- Light Stage system to capture reflectance fields

- Fixed viewpoint, no geometry

- Environment matting [Zongker et al. 99, Chuang et al. 00]

- Capture reflections and refractions

- Fixed viewpoint, no geometry

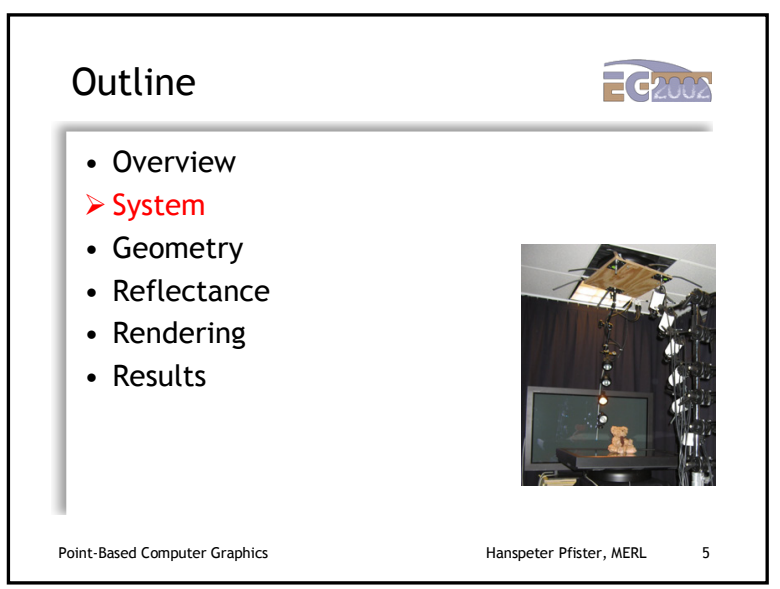

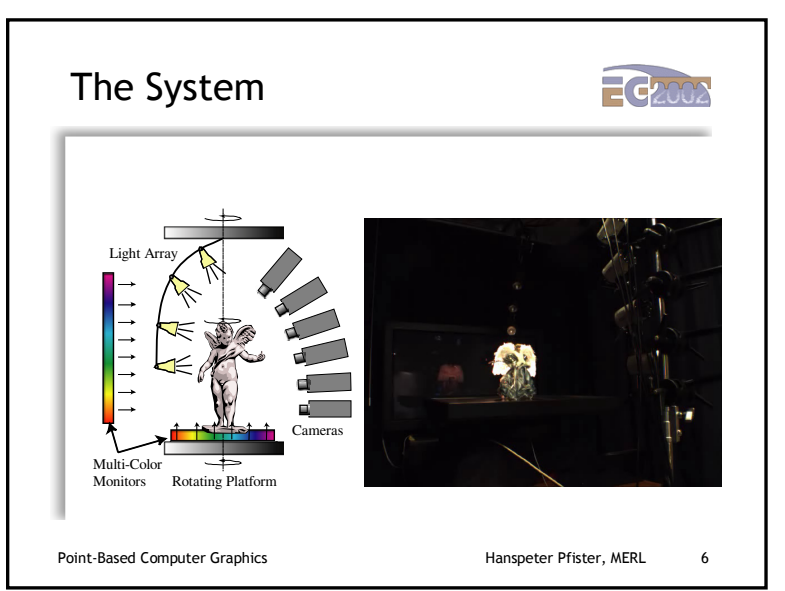



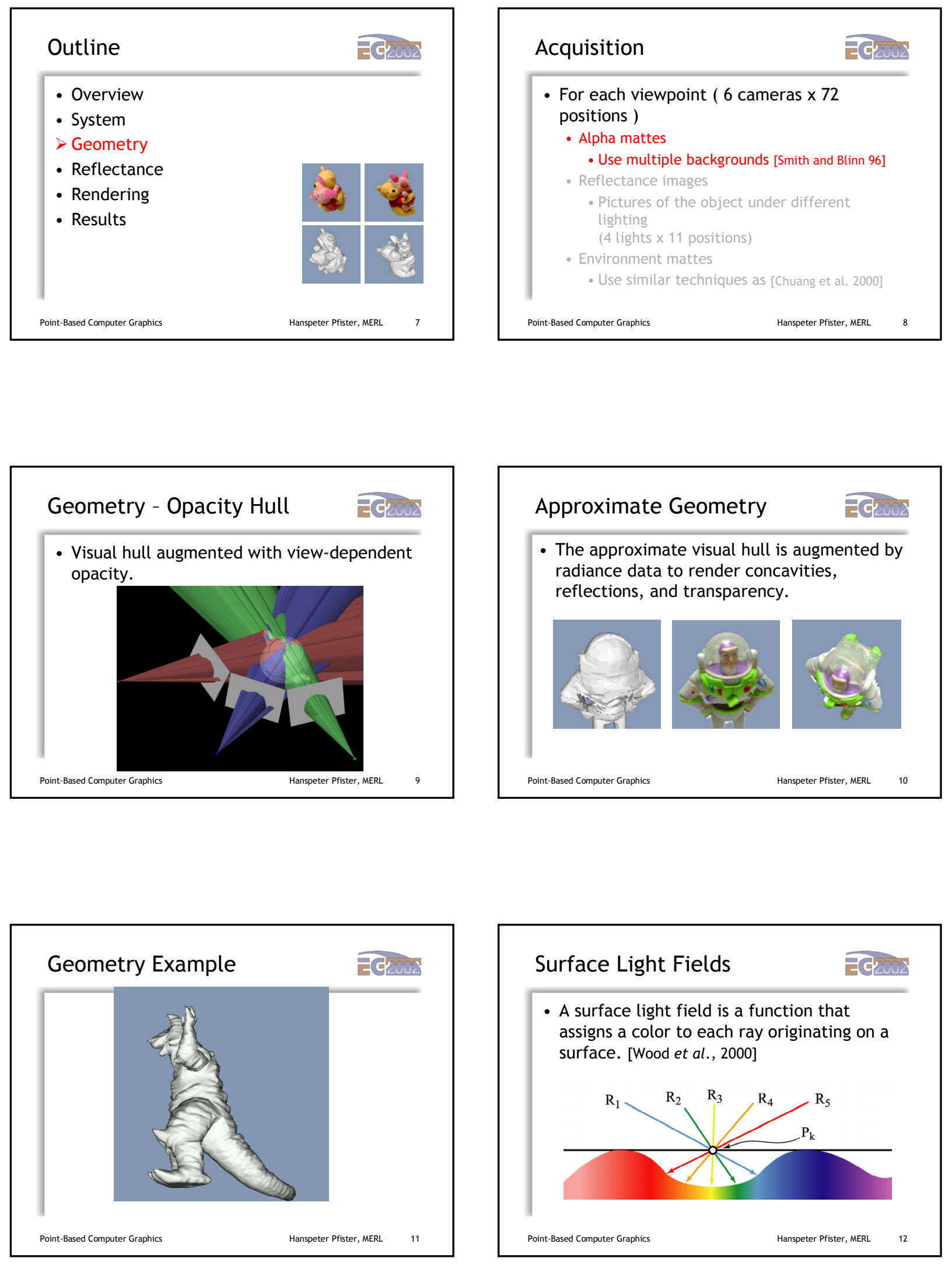

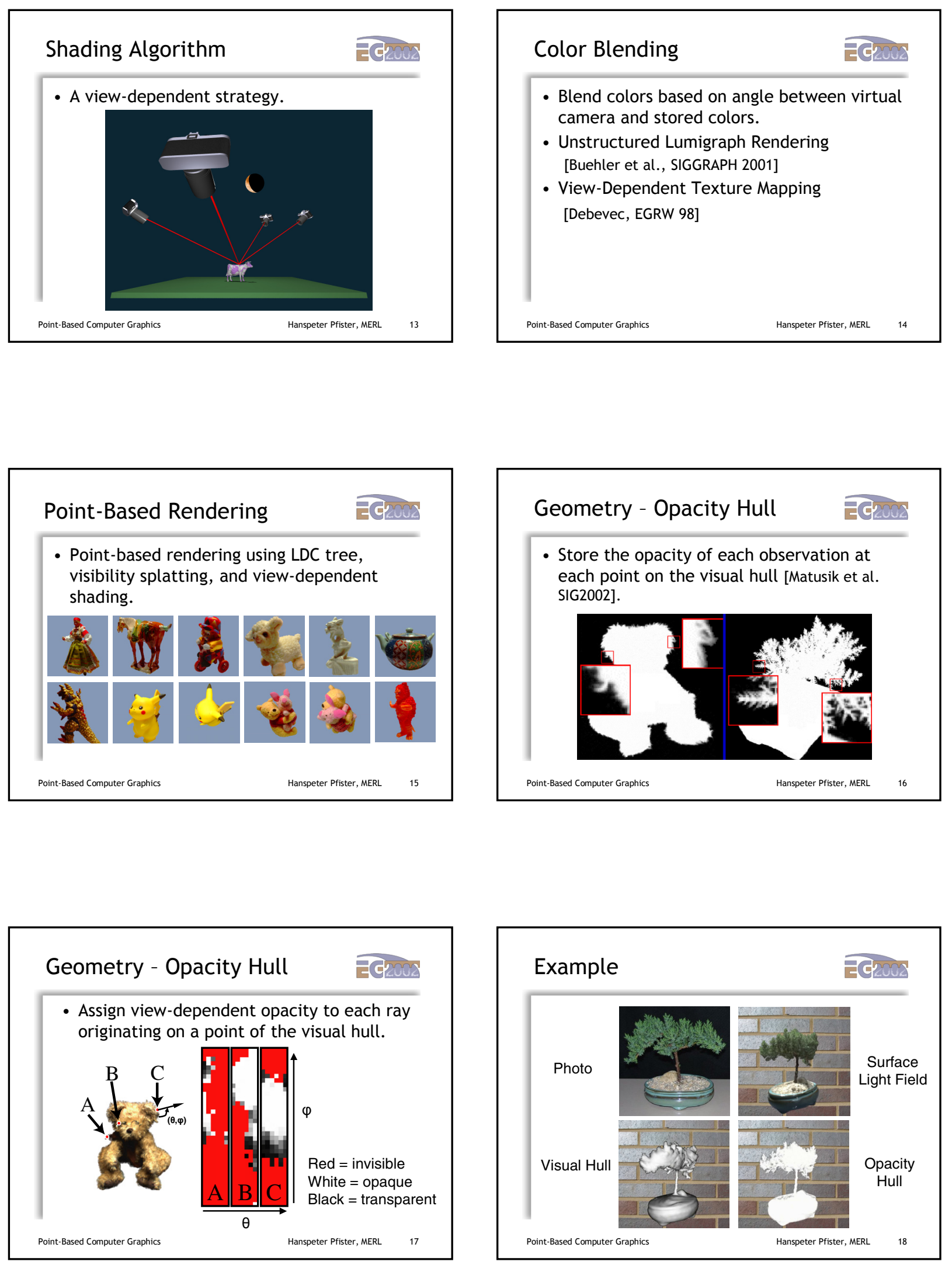

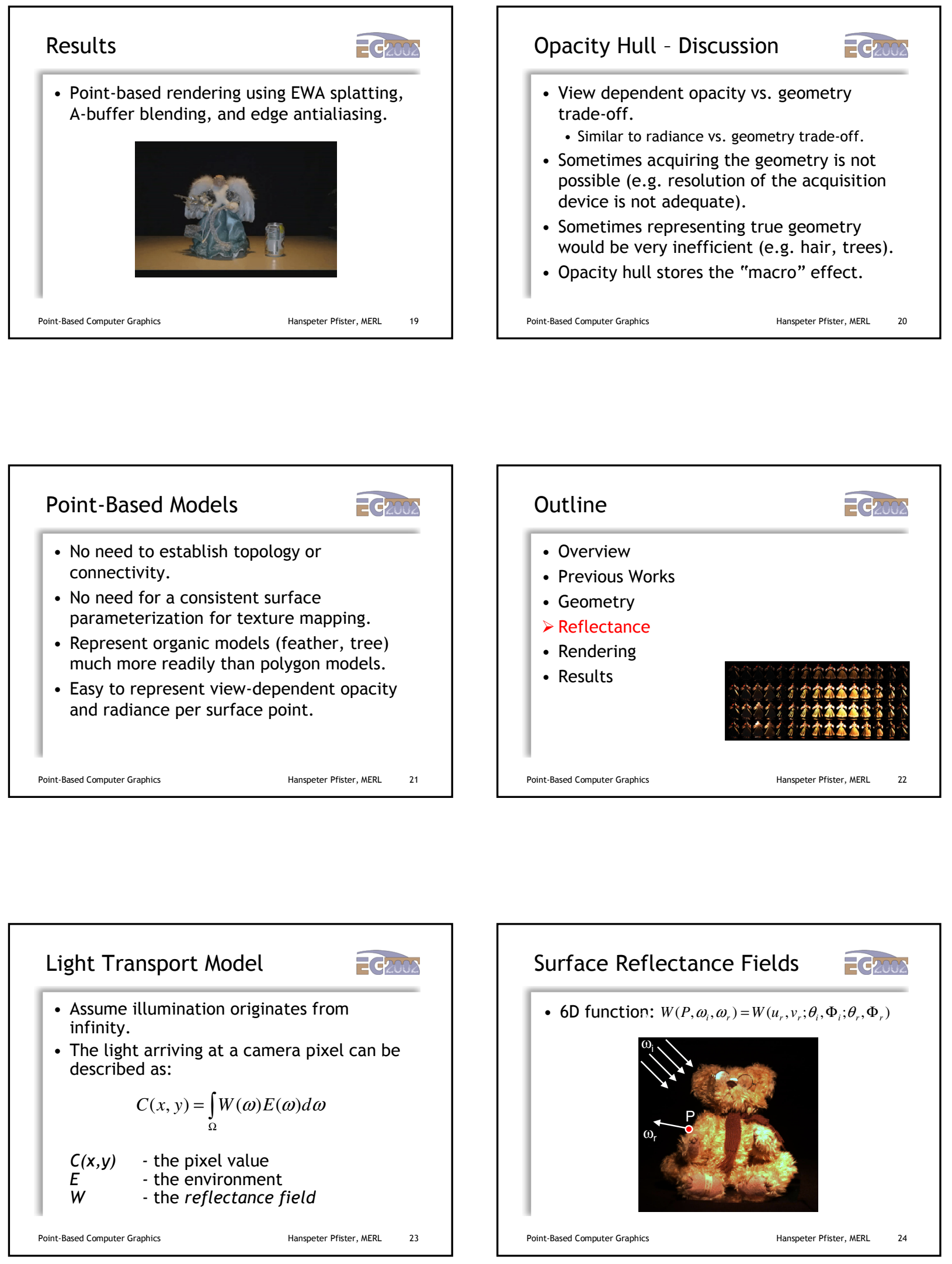

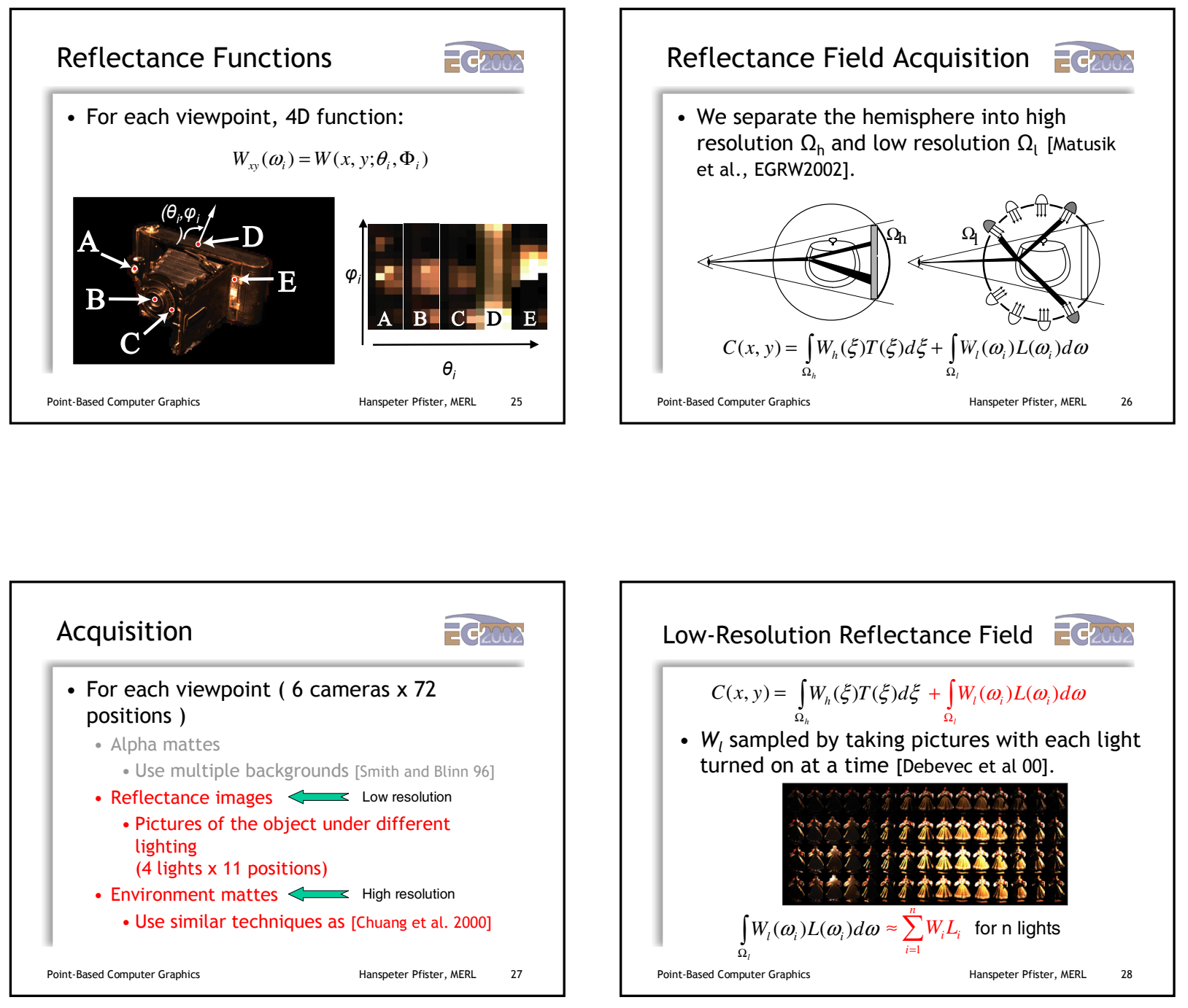

Low-Resolution Reflectance Field =crovs

$C(x, y)=\int_{\Omega_{h}} W_{h}(\xi) T(\xi) d \xi+\int_{\Omega_{l}} W_{l}\left(\omega_{i}\right) L\left(\omega_{i}\right) d \omega$

- $W_{l}$ sampled by taking pictures with each light turned on at a time [Debevec et al 00].

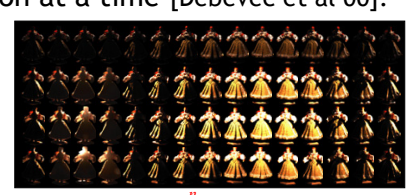

$\int W_{l}\left(\omega_{i}\right) L\left(\omega_{i}\right) d \omega \approx \sum_{i=1}^{n} W_{i} L_{i}$ for $\mathrm{n}$ lights Point-Based Computer Graphics Hanspeter Pfister, MER
High-Resolution Reflectance Field

$$
C(x, y)=\int_{\Omega_{h}} W_{h}(\xi) T(\xi) d \xi+\int_{\Omega_{l}} W_{l}\left(\omega_{i}\right) L\left(\omega_{i}\right) d \omega
$$

- Use techniques of environment matting [Chuang et al., SIGGRAPH 00].

- Approximate $\mathrm{W}_{\mathrm{h}}$ by a sum of up to two Gaussians:

- Reflective $G_{1}$.

- Refractive $G_{2}$.<smiles>CC(C)C(C)(C)C</smiles>
$W_{h}(\xi)=a_{1} G_{1}+a_{2} G_{2}$

Point-Based Computer Graphics Hanspeter Pfister, MERL $\quad 30$ 


\section{Surface Reflectance Fields

- Work without accurate geometry.

- Surface normals are not necessary.

- Capture more than reflectance:

- Inter-reflections

- Subsurface scattering

- Refraction

- Dispersion

- Non-uniform material variations

- Simplified version of the BSSRDF [Debevec et al., 00].
Outline

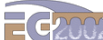

- Overview

- Previous Works

- Geometry

- Reflectance

$>$ Rendering

- Results

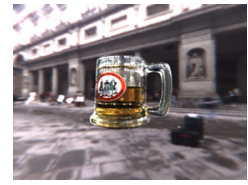

\section{Rendering}

\section{다일}

- Input: Opacity hull, reflectance data, new environment

- Create radiance images from environment and low-resolution reflectance field.

- Reparameterize environment mattes.

- Interpolate data to new viewpoint. $1^{\text {st }}$ Step: Relighting $\Omega_{l}$

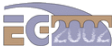

- Compute radiance image for each viewpoint.

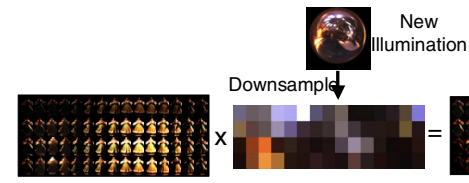

The sum is the radiance image of this viewpoint in this environment.

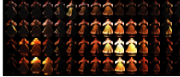

4

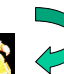
Hanspeter Pfister, MER

\section{$2^{\text {nd }}$ Step: Reproject $\Omega_{\mathrm{h}}$}

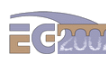

- Project environment mattes onto the new environment.

- Environment mattes acquired was parameterized on plane T (the plasma display).

- We need to project the Gaussians to the new environment map, producing new Gaussians.
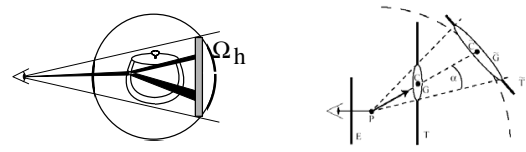

\section{$3^{\text {rd }}$ Step: Interpolation}

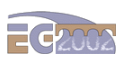

- From new viewpoint, for each surface point, find four nearest acquired viewpoints.

- Store visibility vector per surface point.

- Interpolate using unstructured lumigraph interpolation [Buehler et al., SIGGRAPH 01] or viewdependent texture mapping [Debevec 96].

- Opacity.

- Contribution from low-res reflectance field (in the form of

radiance images).

- Contribution from high-res reflectance field. 


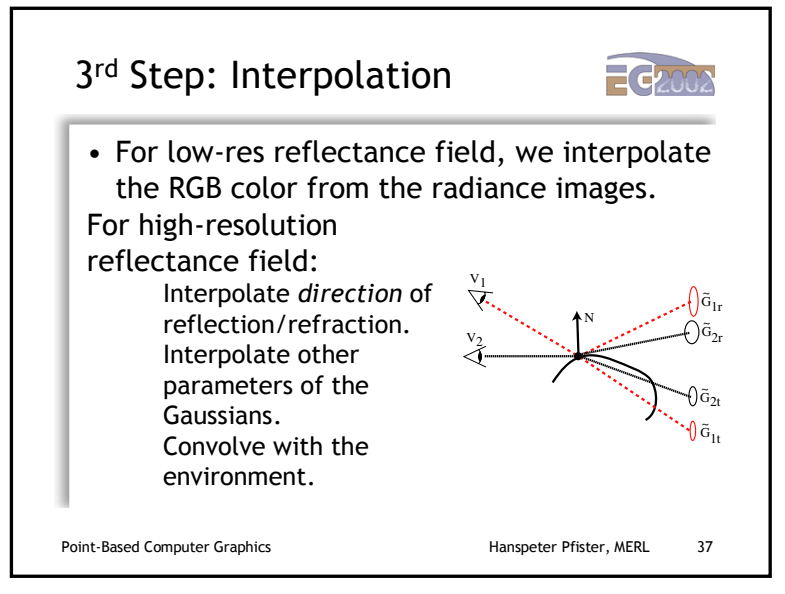

\begin{tabular}{|l|l|}
\hline Outline & \\
\hline - Overview \\
- Previous Works \\
- Geometry \\
- Reflectance \\
- Rendering \\
$>$ Results \\
\\
\end{tabular}
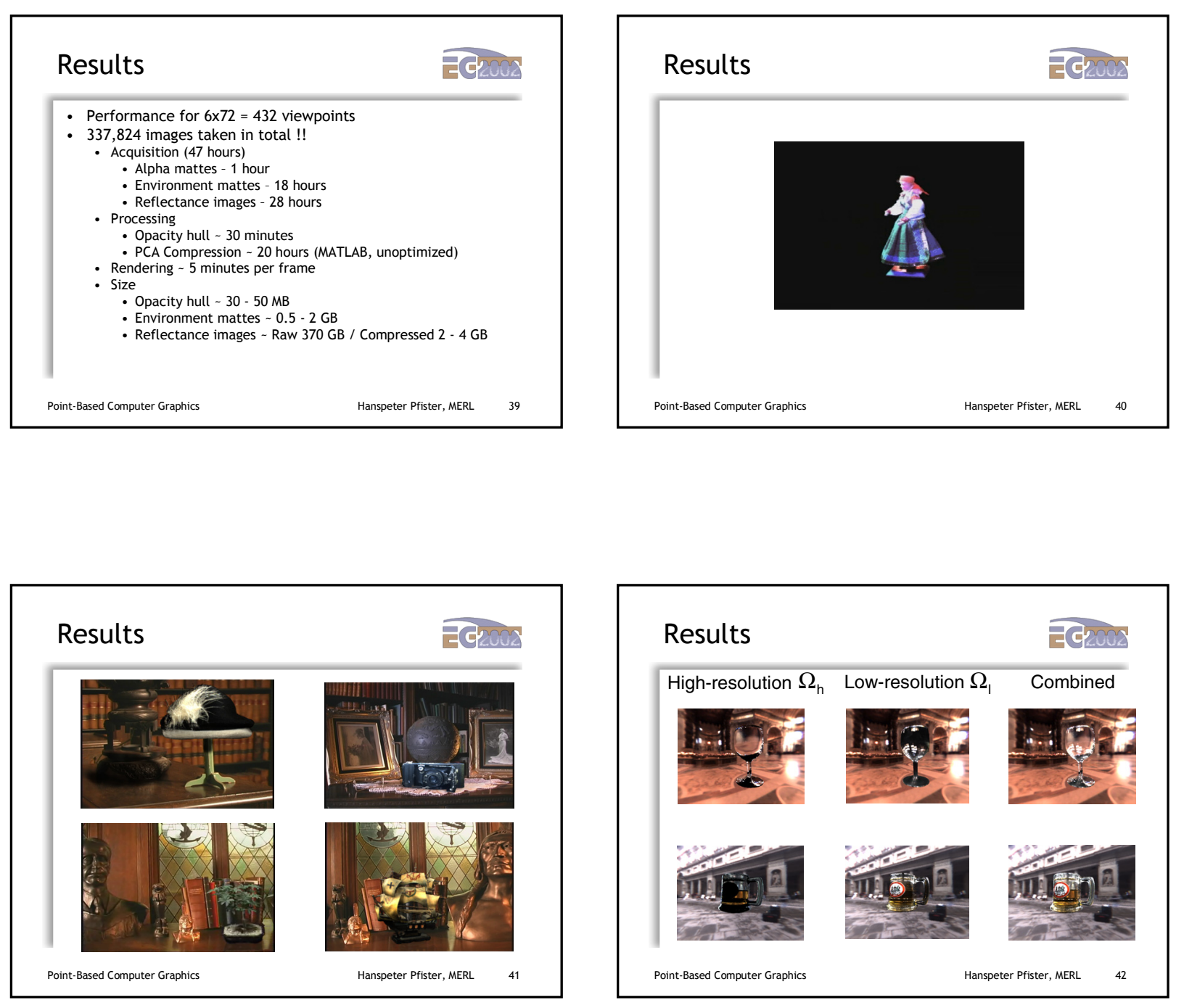

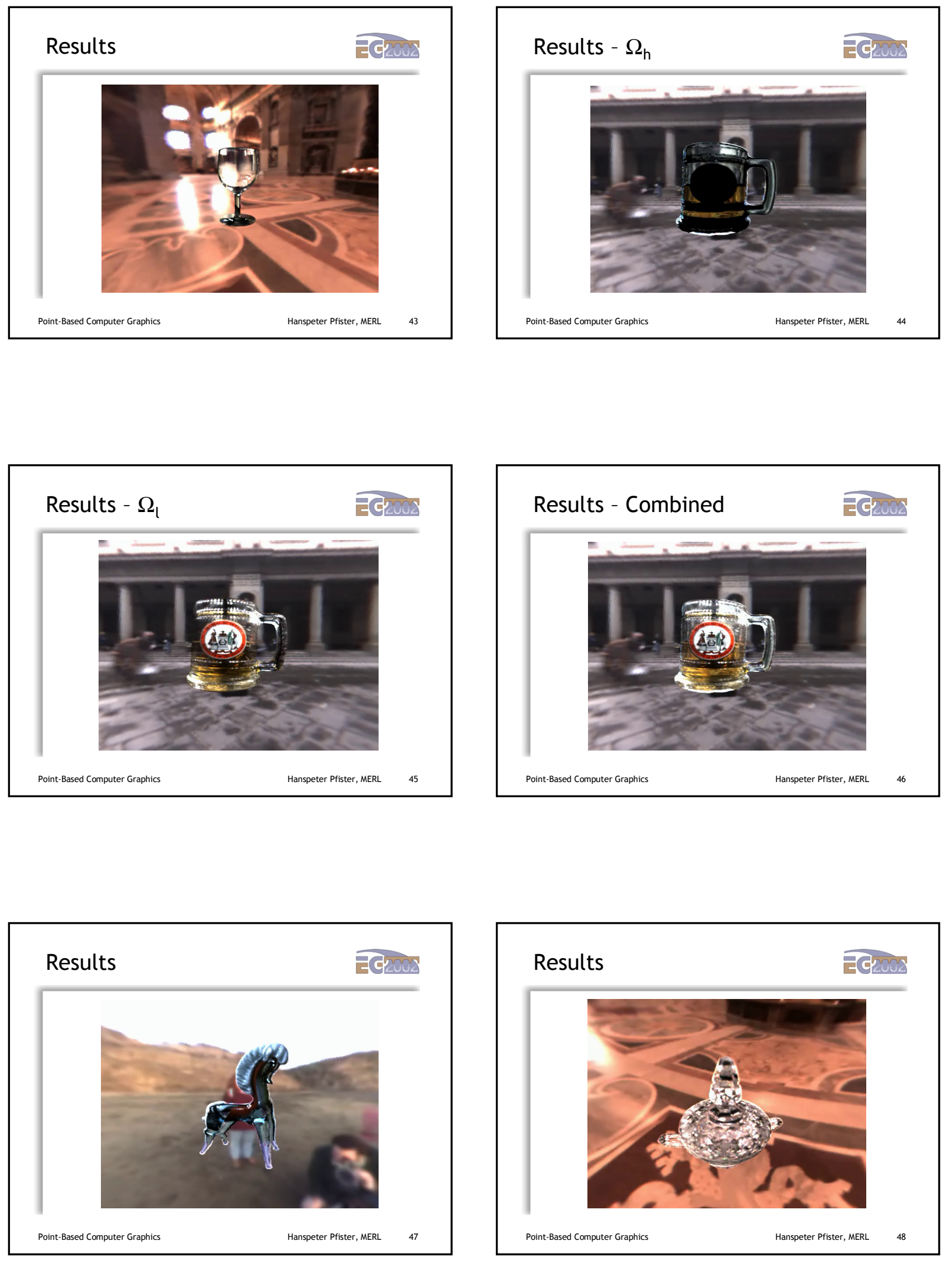

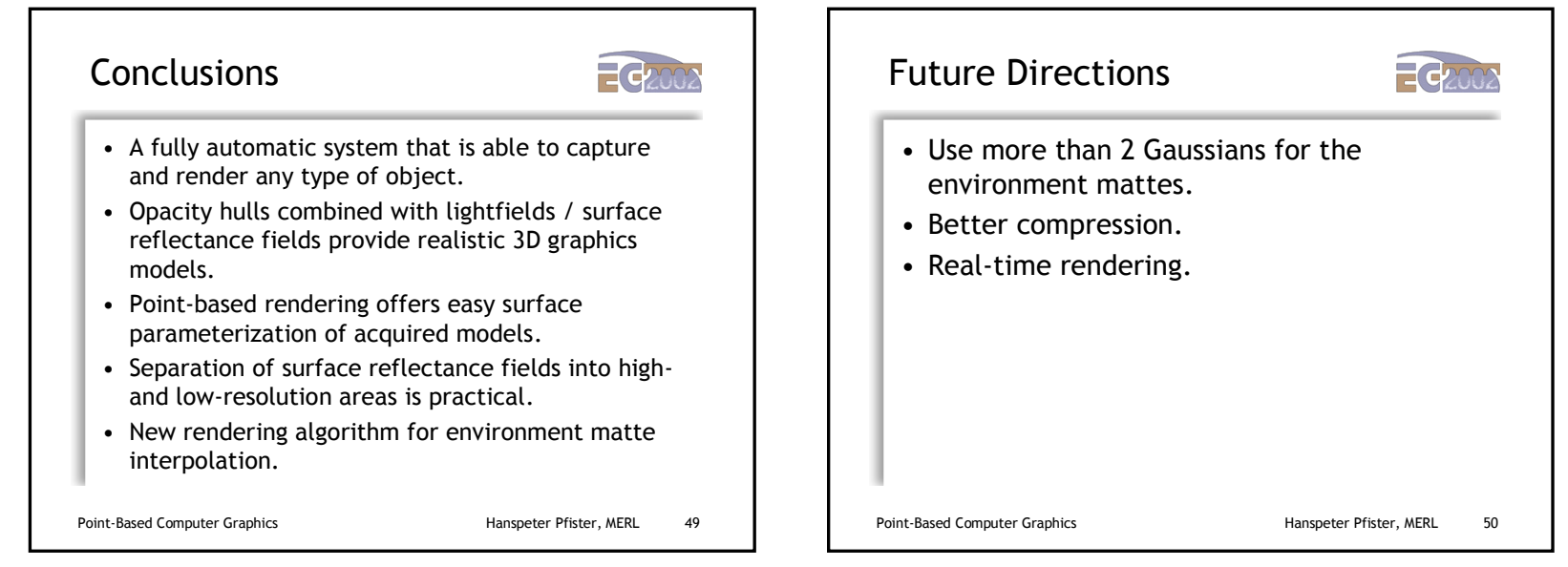

\section{Acknowledgements}

- Colleagues:

- MIT: Chris Buehler, Tom Buehler.

- MERL: Bill Yerazunis, Darren Leigh, Michael

Stern.

- Thanks to:

- David Tames, Jennifer Roderick Pfister.

- NSF grants CCR-9975859 and EIA-9802220.

- Papers available at:

- http://www.merl.com/people/pfister/ 

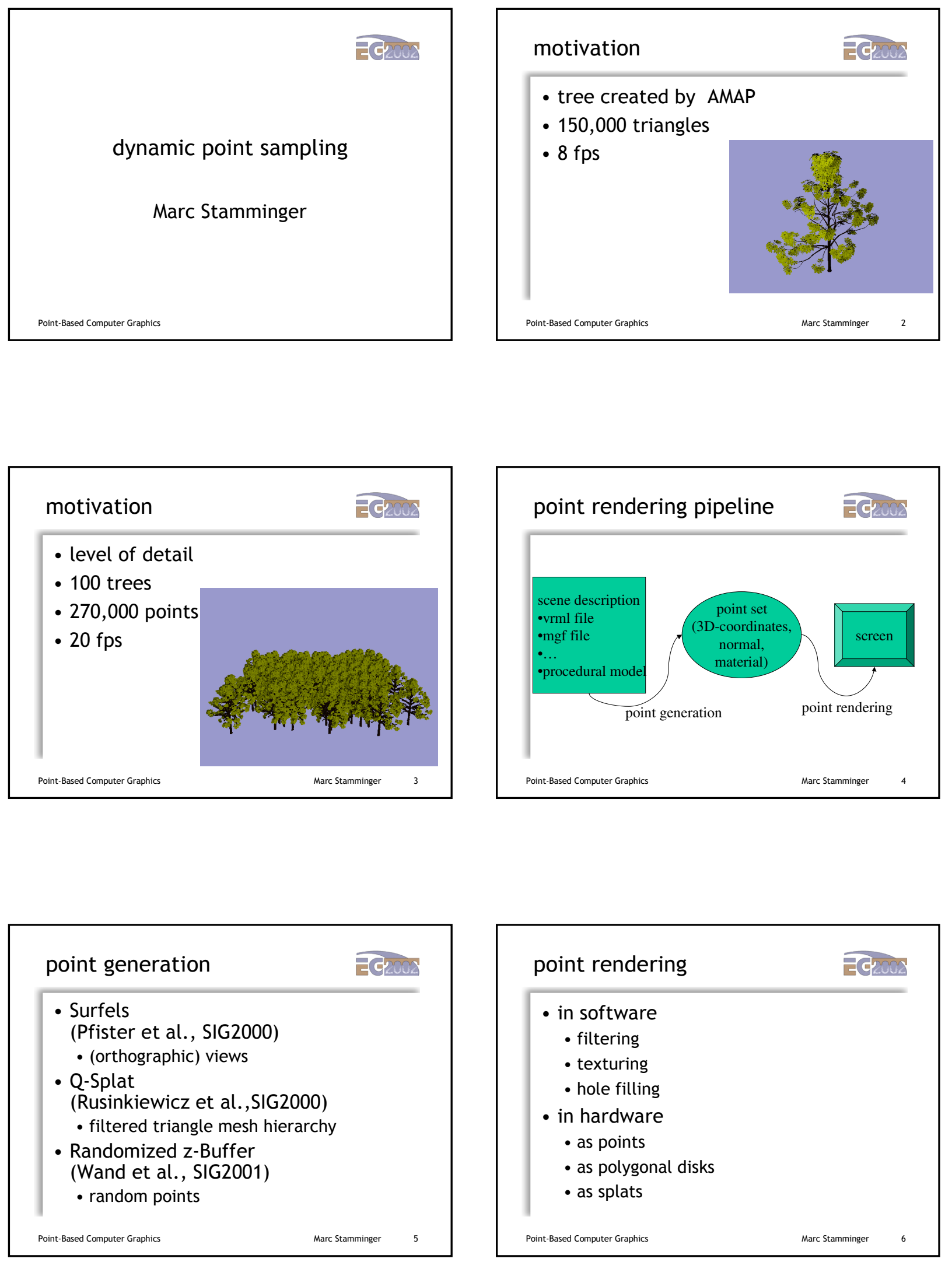

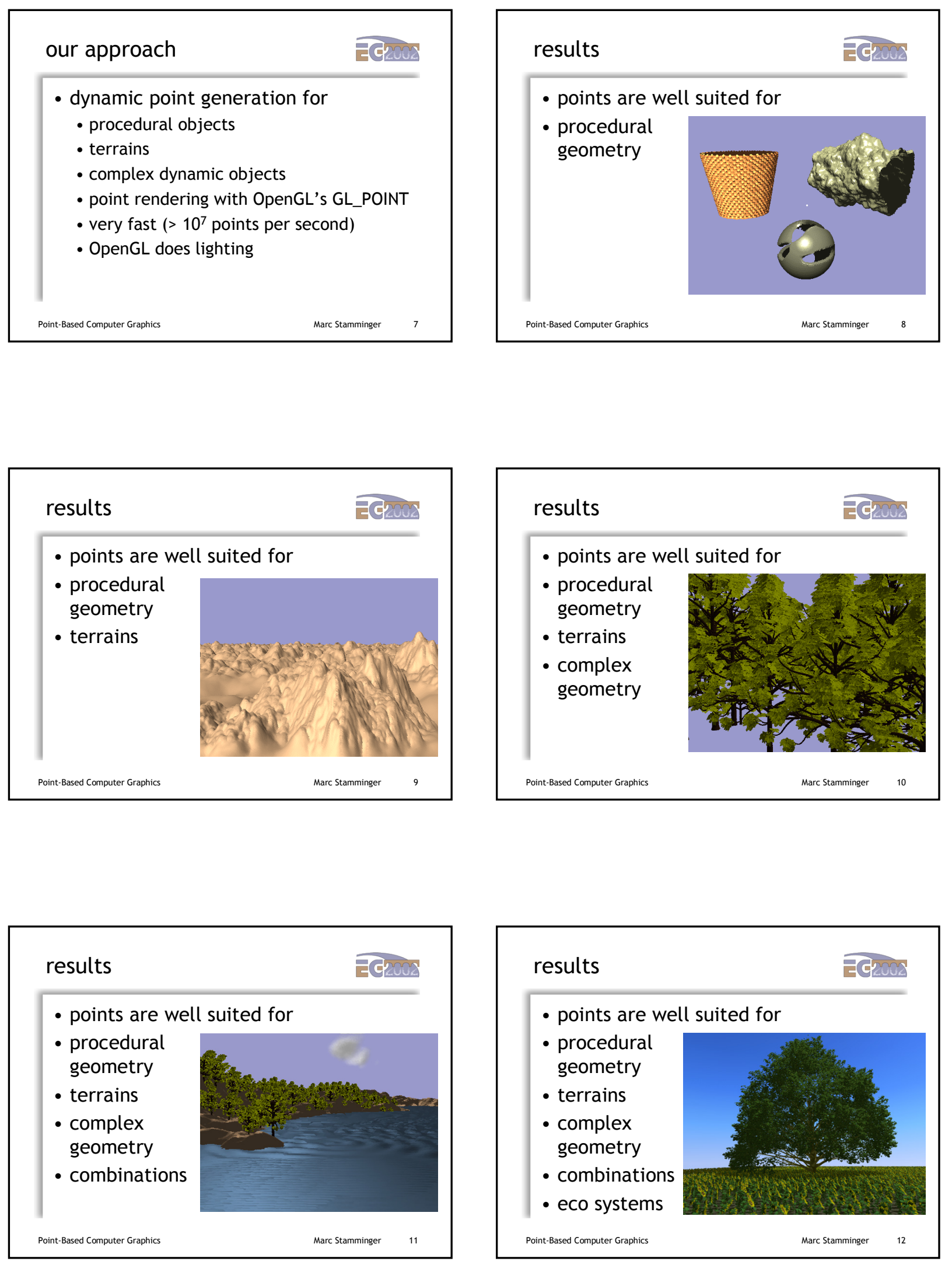

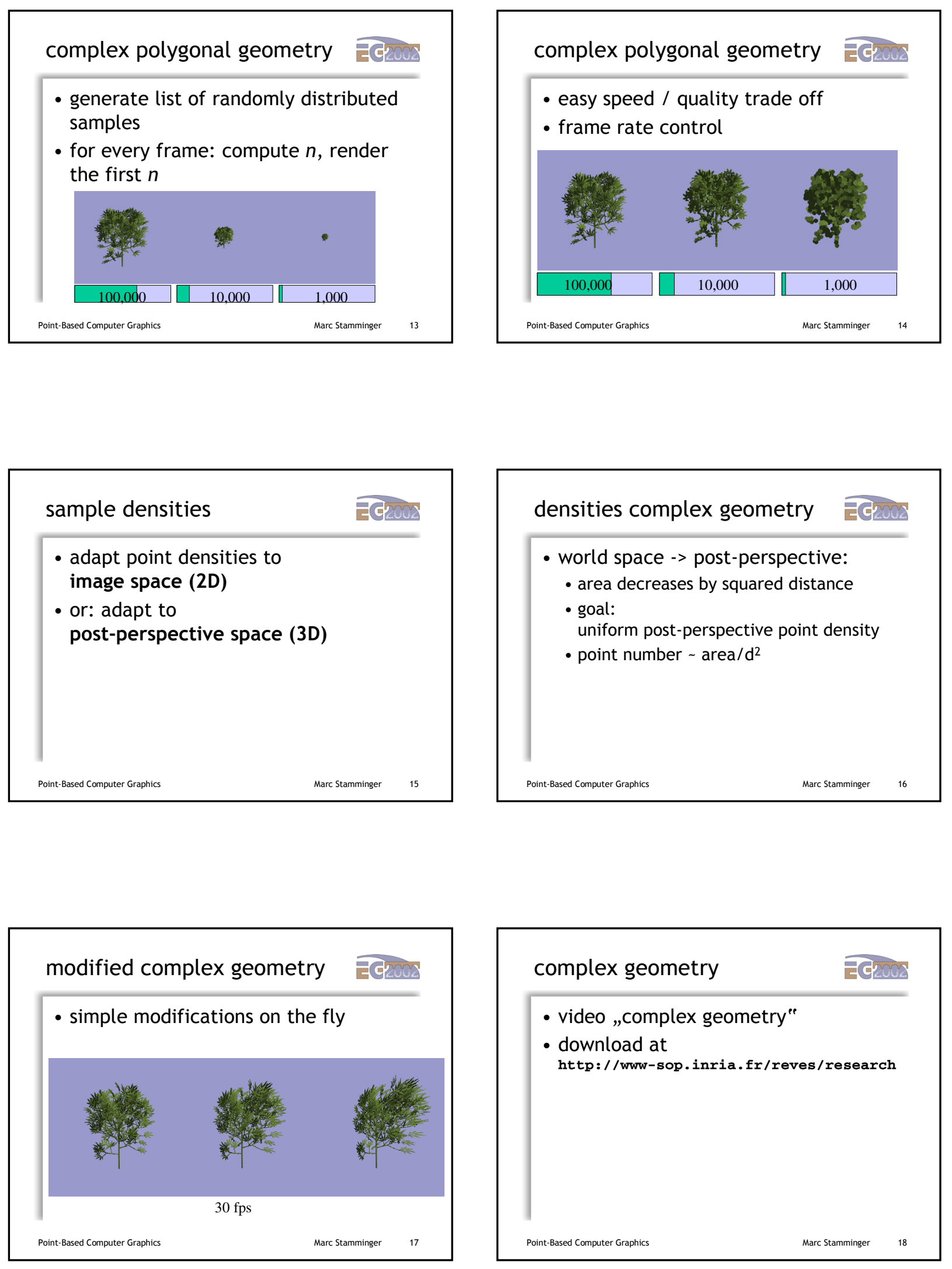

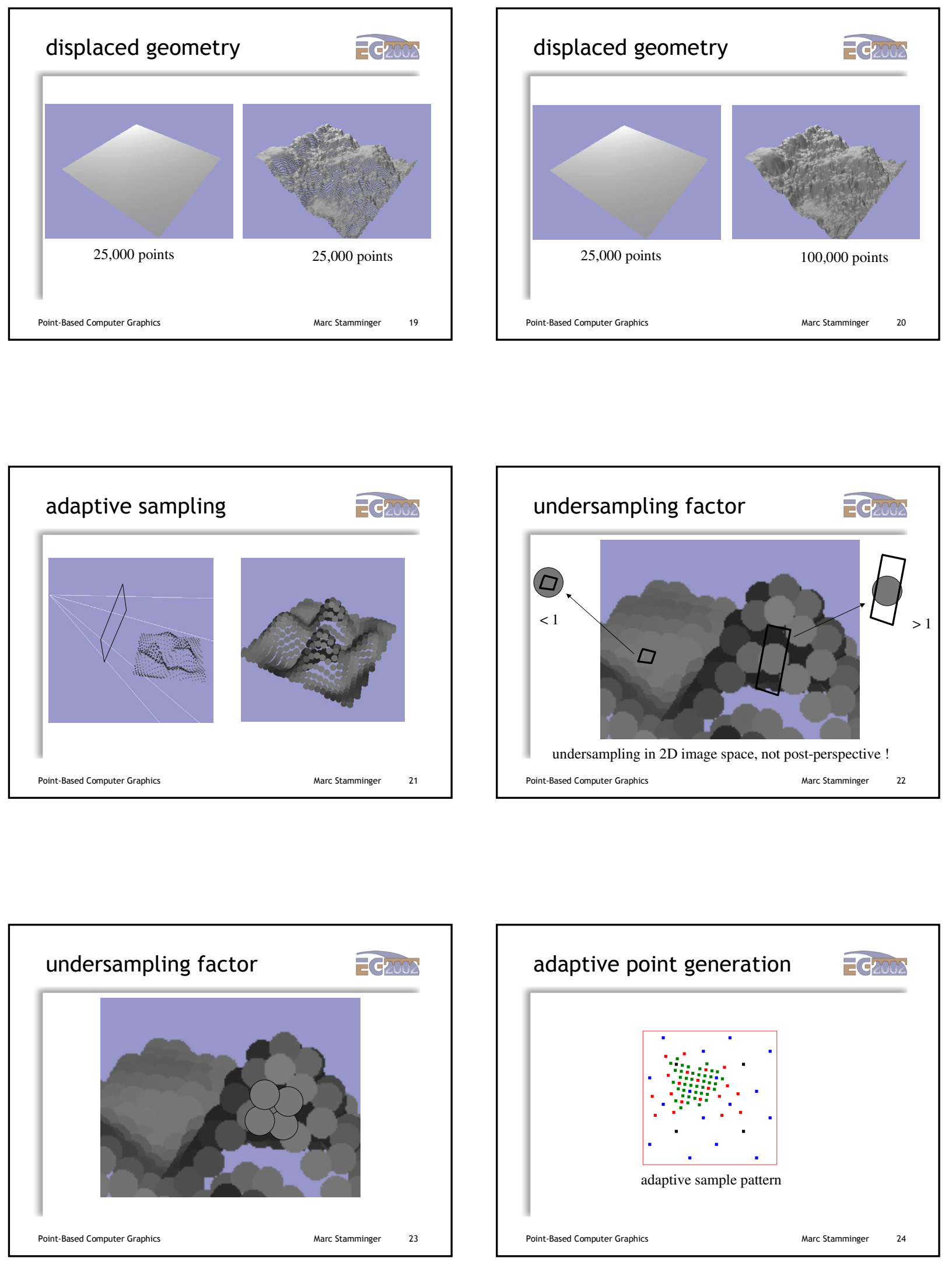


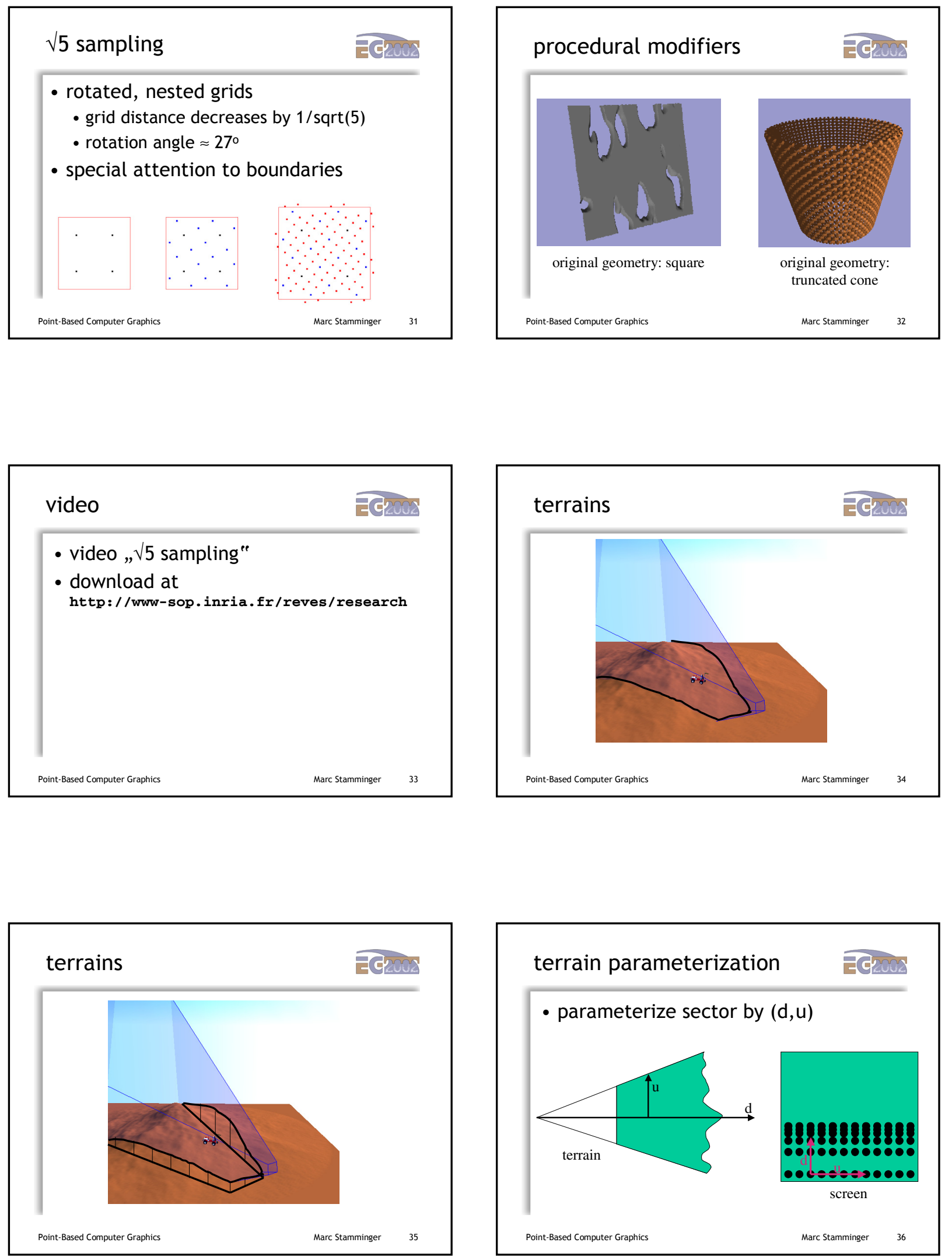

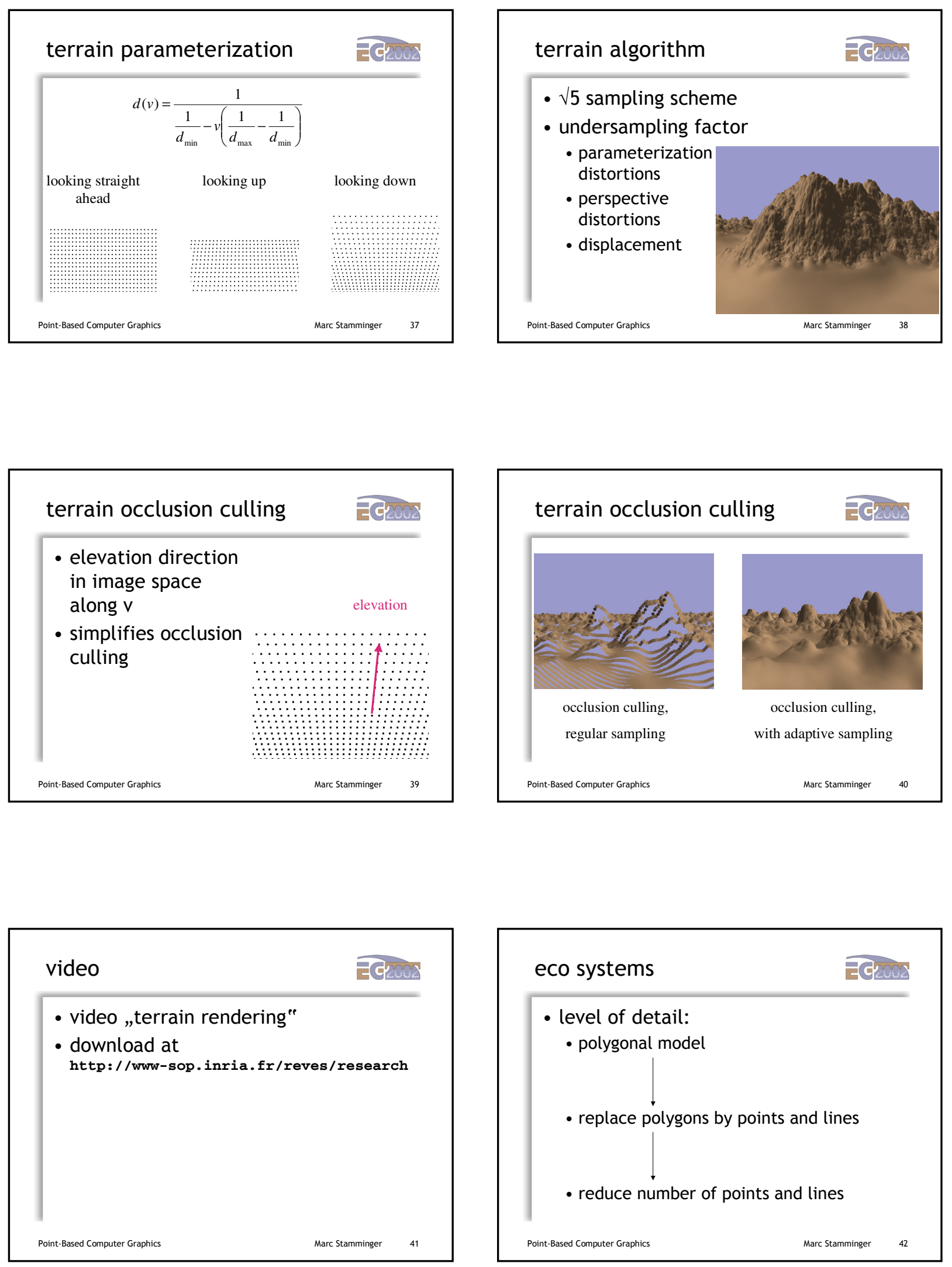

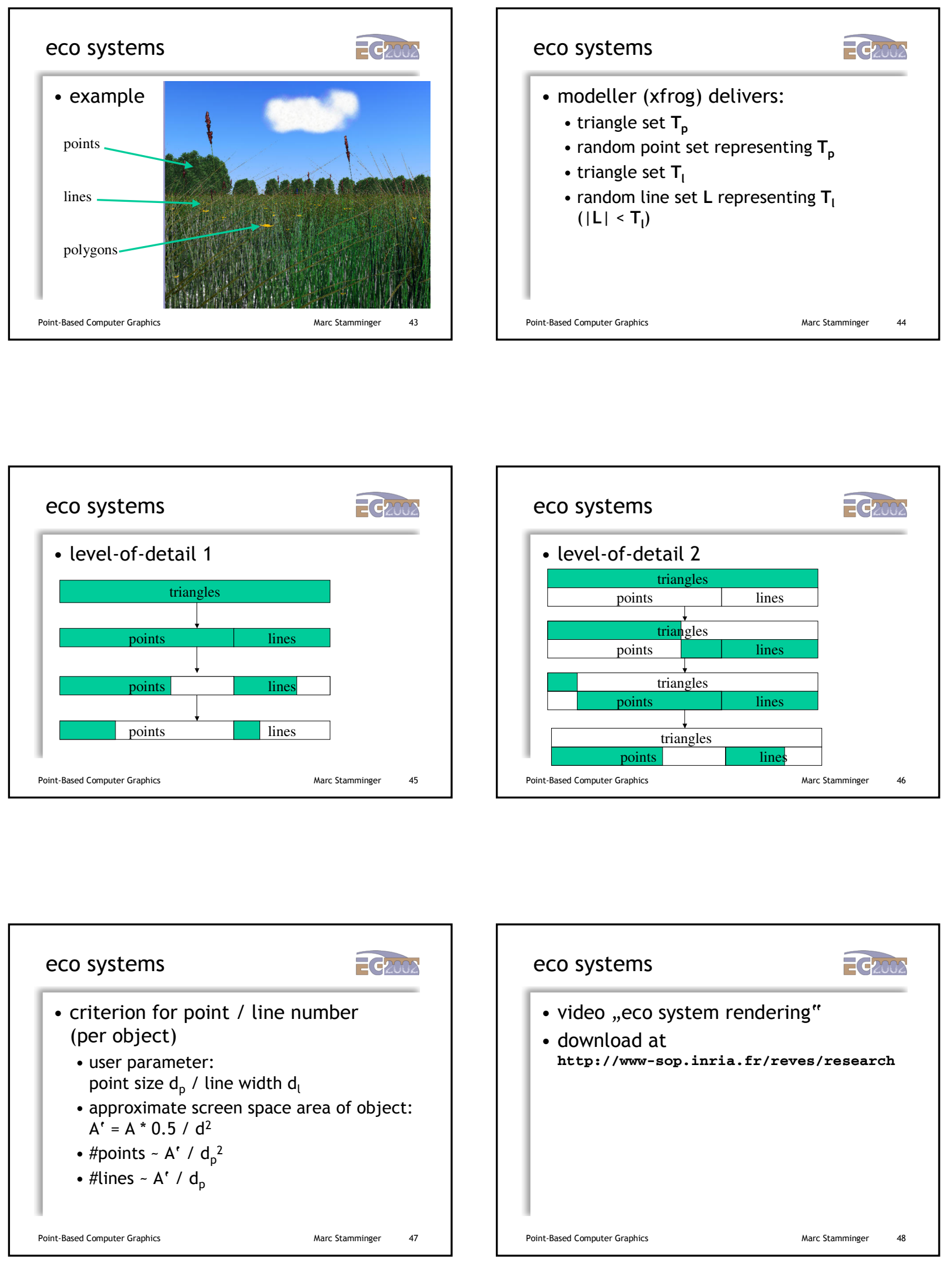


\begin{tabular}{|c|}
\hline Surfaces from Point Samples \\
Marc Alexa \\
TU Darmstadt \\
\\
Ponint-Bseded computer Graphics
\end{tabular}
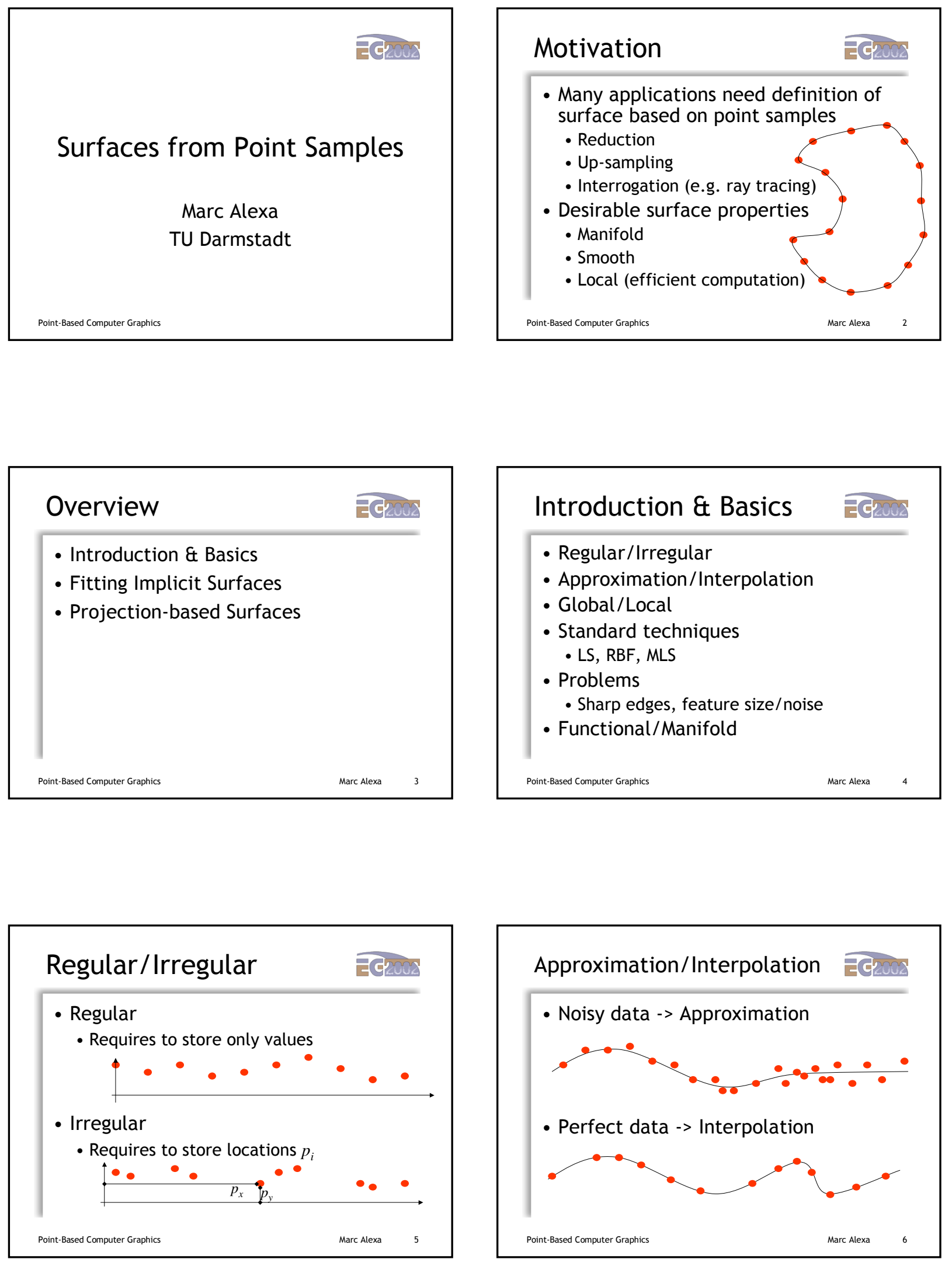

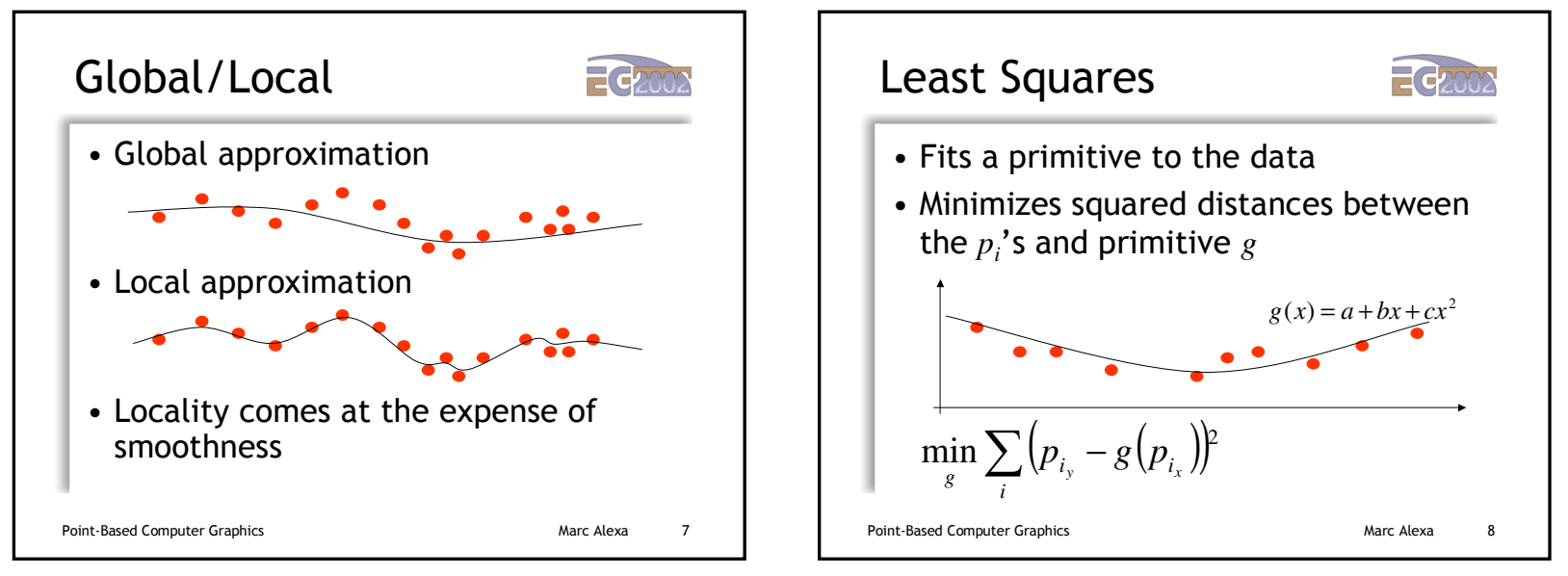

\section{Least Squares - Example EArowas}

- Primitive is a polynomial

$$
\begin{gathered}
g(x)=\left(1, x, x^{2}, \ldots\right) \cdot \mathbf{c}^{T} \\
\cdot \min \sum_{i}\left(p_{i_{y}}-\left(1, p_{i_{x}}, p_{i_{x}}^{2}, \ldots\right) \mathbf{c}^{T}\right)^{2} \Rightarrow \\
0=\sum_{i} 2 p_{i_{x}}^{j}\left(p_{i_{y}}-\left(1, p_{i_{x}}, p_{i_{x}}^{2}, \ldots\right) \mathbf{c}^{T}\right)
\end{gathered}
$$

- Linear system of equations

\section{Moving Least Squares}

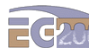

- Compute a local LS approximation at $t$

- Weight data points based on distance to $t$

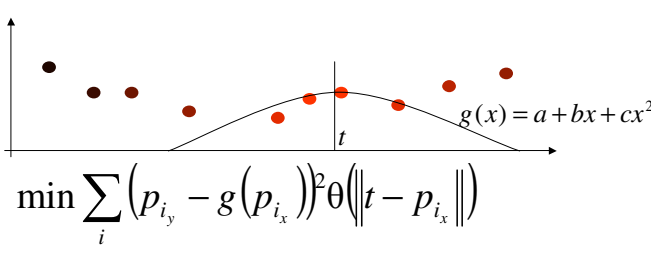

Point-Based Computer Graphics

Marc Alexa
Moving Least Squares

\section{Iacroon}

- The set

$f(t)=g_{t}(t), g_{t}: \min _{g} \sum_{i}\left(p_{i_{y}}-g\left(p_{i_{x}}\right)\right)^{2} \theta\left(\left\|t-p_{i_{x}}\right\|\right)$

is a smooth curve, iff $\theta$ is smooth

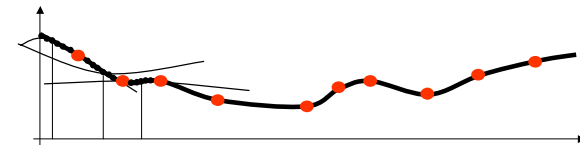




\section{Moving Least Squares}

- Typical choices for $\theta$ :

- $\theta(d)=d^{-r}$

- $\theta(d)=e^{-d^{2} / h^{2}}$

- Note: $\theta_{i}=\theta\left(\left\|t-p_{i_{x}}\right\|\right)$ is fixed

- For each $t$

- Standard weighted LS problem

- Linear iff corresponding LS is linear

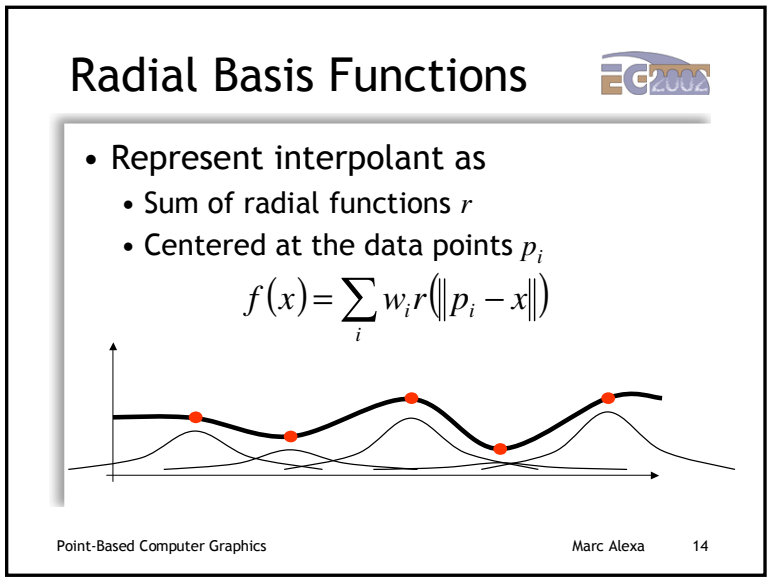

\section{Radial Basis Functions}

\section{$=0$}

- Solvability depends on radial function

- Several choices assure solvability

- $r(d)=d^{2} \log d \quad$ (thin plate spline)

- $r(d)=e^{-d^{2} / h^{2}} \quad$ (Gaussian)

- $h$ is a data parameter

- $h$ reflects the feature size or anticipated spacing among points

\section{Typical Problems}

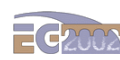

- Sharp corners/edges

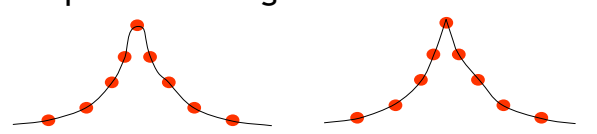

- Noise vs. feature size
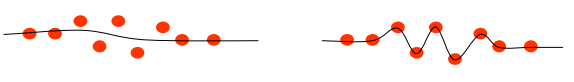

Point-Based Computer Graphics

\section{Functional/Manifold}

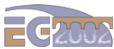

- Standard techniques are applicable if data represents a function

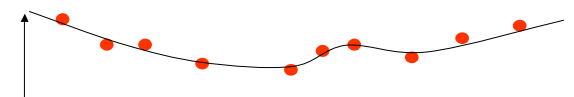

- Manifolds are more general

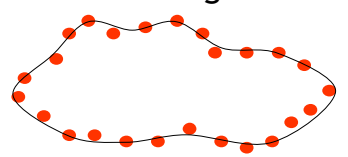

Point-Based Computer Graphics 

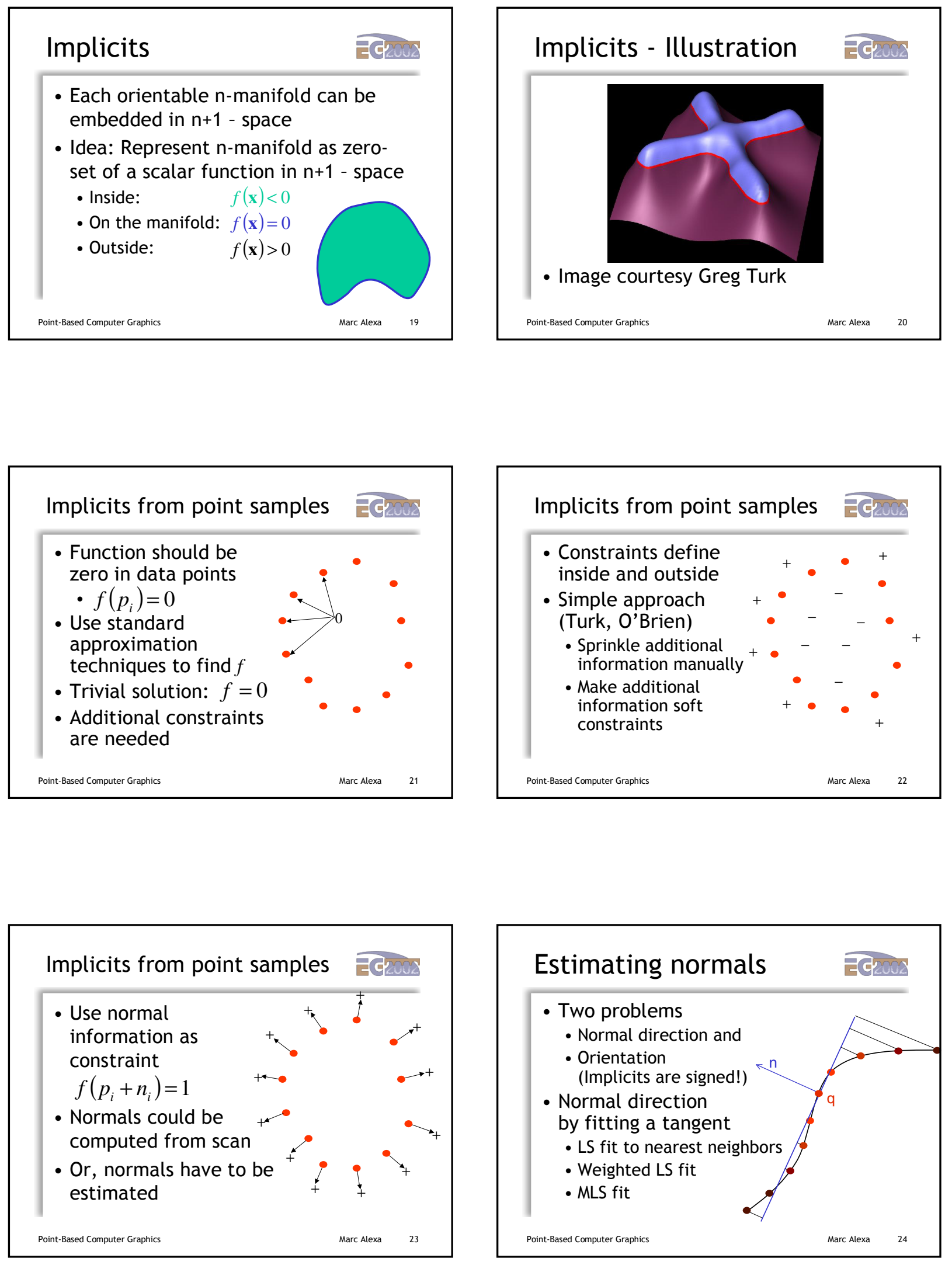

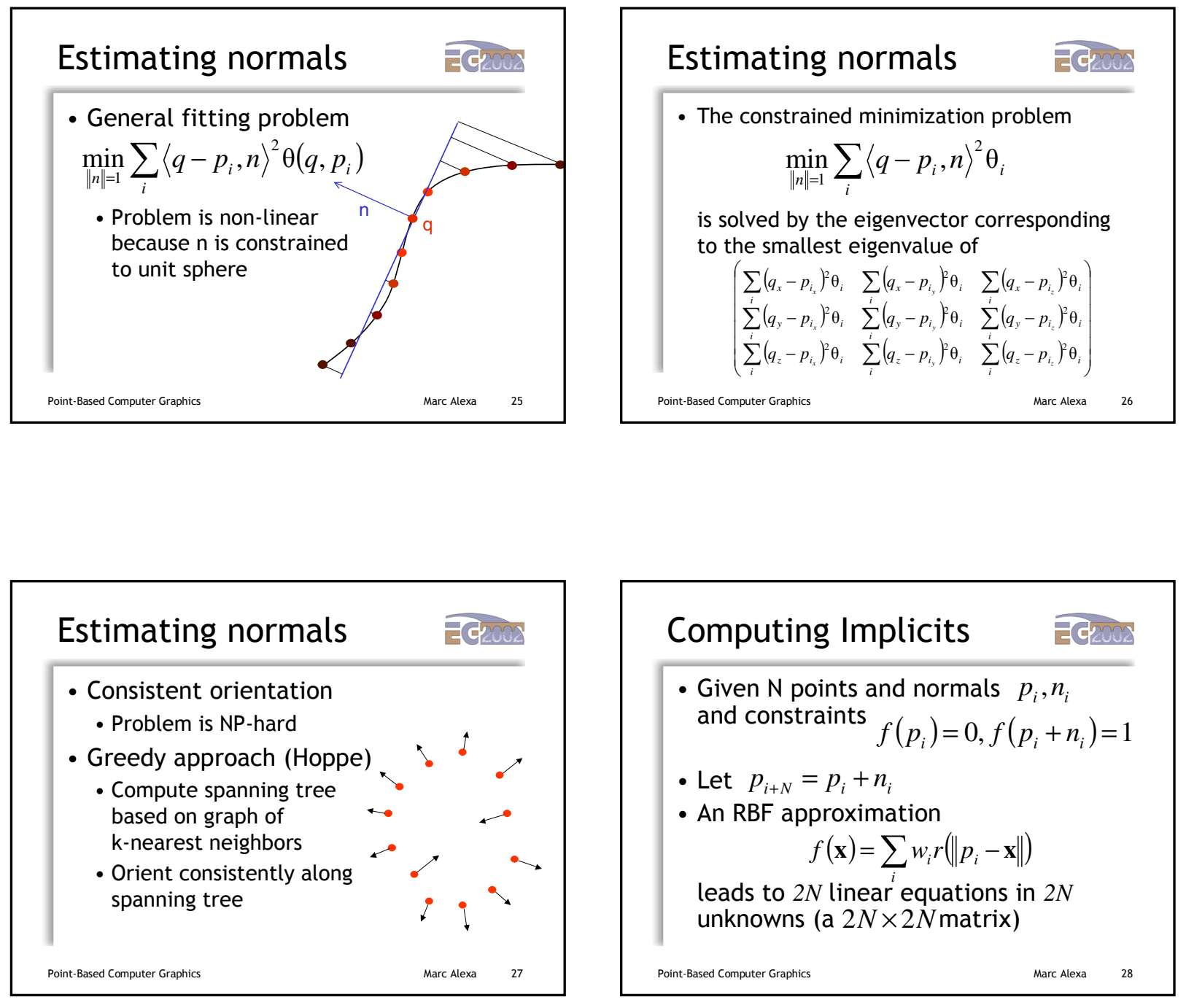

\section{Computing Implicits}

\section{actors}

- Given $\mathrm{N}$ points and normals $p_{i}, n_{i}$ and constraints

$$
f\left(p_{i}\right)=0, f\left(p_{i}+n_{i}\right)=1
$$

- Let $p_{i+N}=p_{i}+n_{i}$

- An RBF approximation

$$
f(\mathbf{x})=\sum_{i} w_{i} r\left(\left\|p_{i}-\mathbf{x}\right\|\right)
$$

leads to $2 N$ linear equations in $2 N$ unknowns (a $2 N \times 2 N$ matrix)

\section{Computing Implicits}

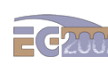

- Practical problems: $N>10000$

- Matrix solution becomes difficult

- Two solutions

- Sparse matrices allow iterative solution

- Smaller number of RBFs

\section{Computing Implicits}

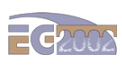

- Sparse matrices $\left(\begin{array}{rrr}r(0) & r\left(\left\|p_{0}-p_{1}\right\|\right) & r\left(\left\|p_{0}-p_{2}\right\|\right)\end{array}\right.$

$$
\begin{array}{ccc}
r\left(\left\|p_{1}-p_{0}\right\|\right) & r(0) & r\left(\left\|p_{1}-p_{2}\right\|\right) \\
r\left(\left\|p-p_{0}\right\|\right) & r\left(\left\|p_{2}-p_{1}\right\|\right) & r(0)
\end{array}
$$

- Needed: $d>c \rightarrow r(d)=0, r^{\prime}(c)=0$

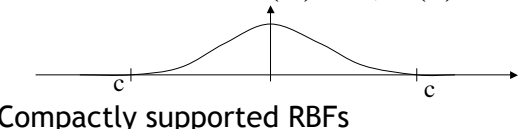

- Compactly supported RBFs 

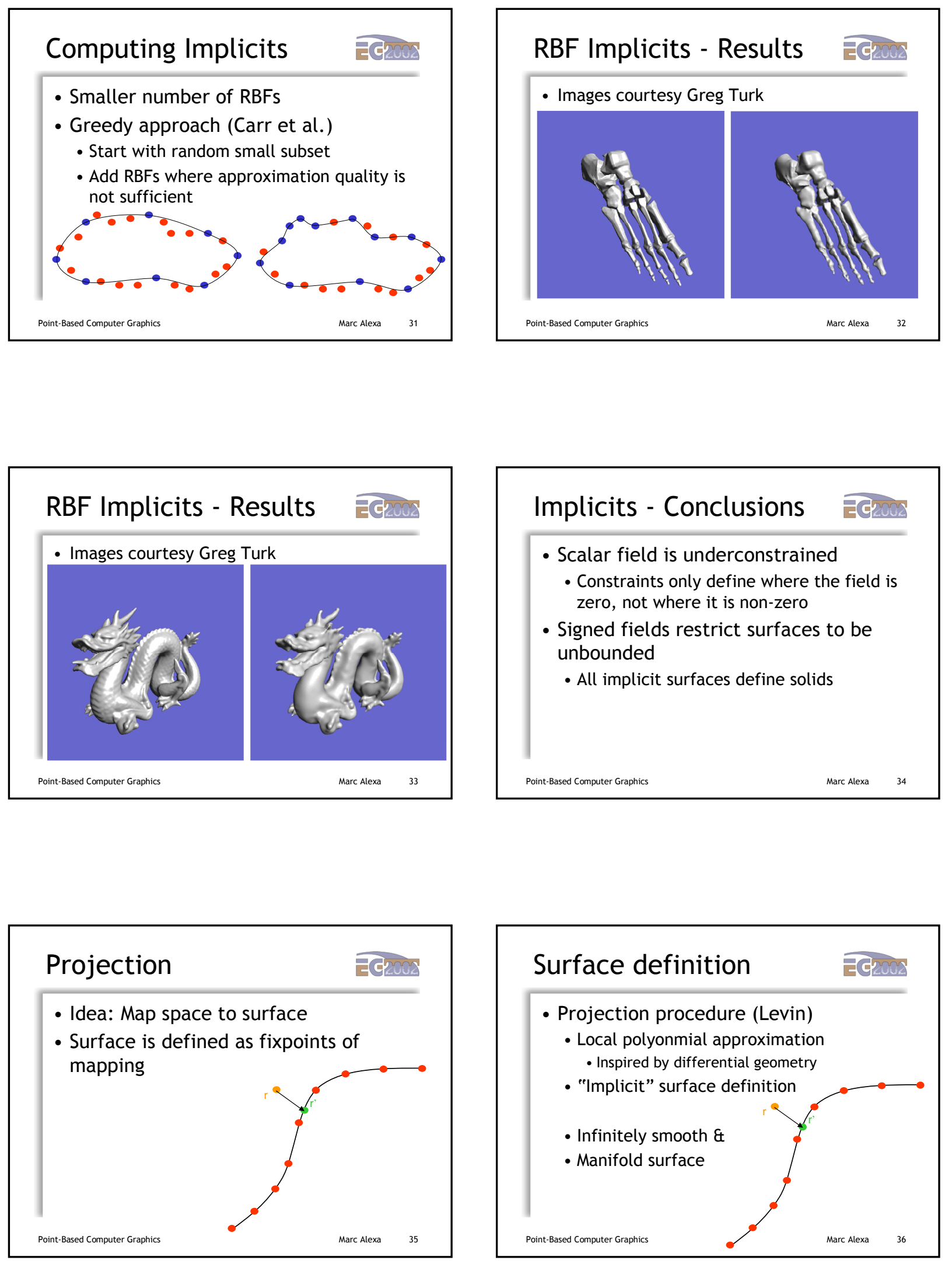

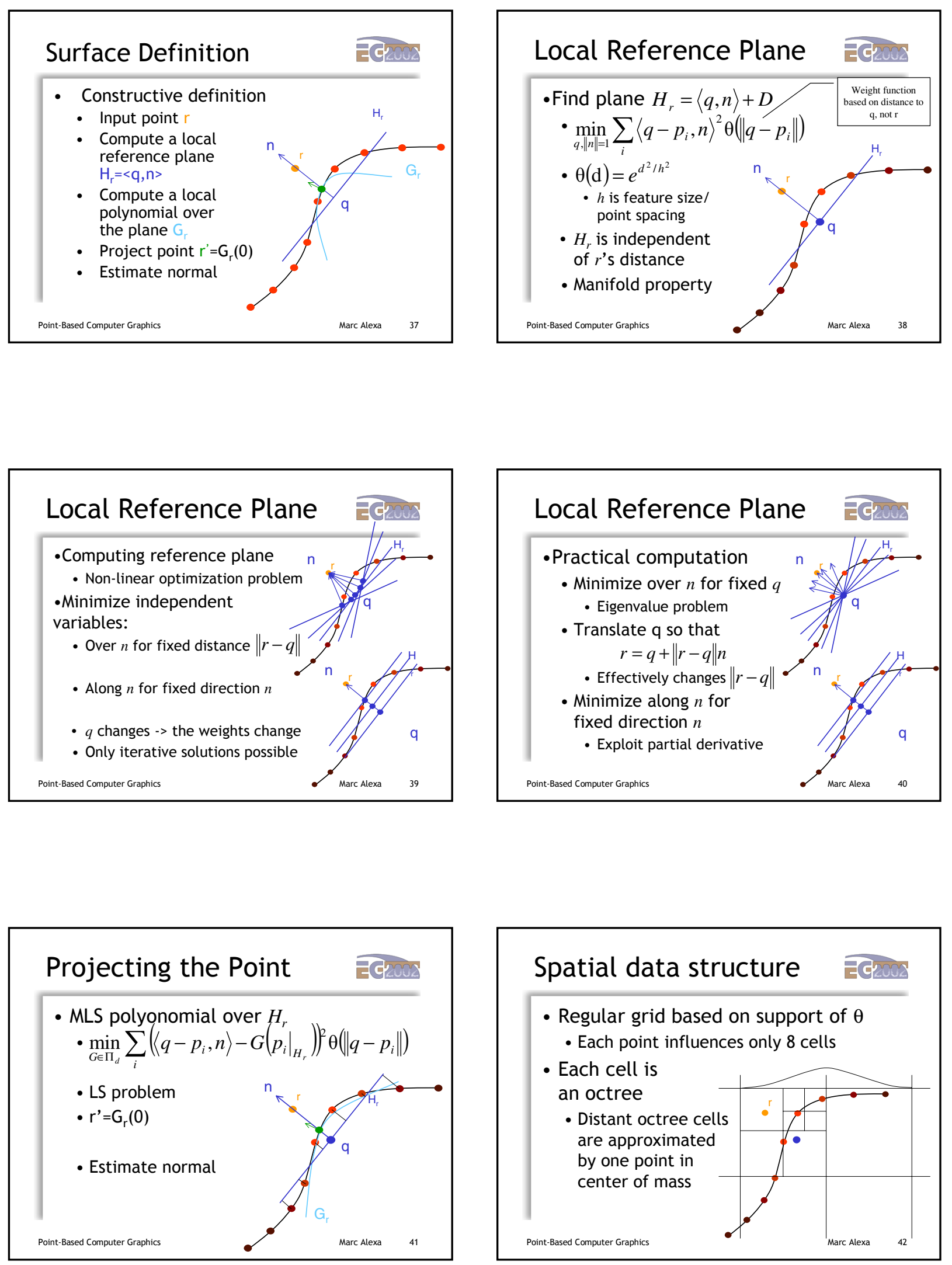


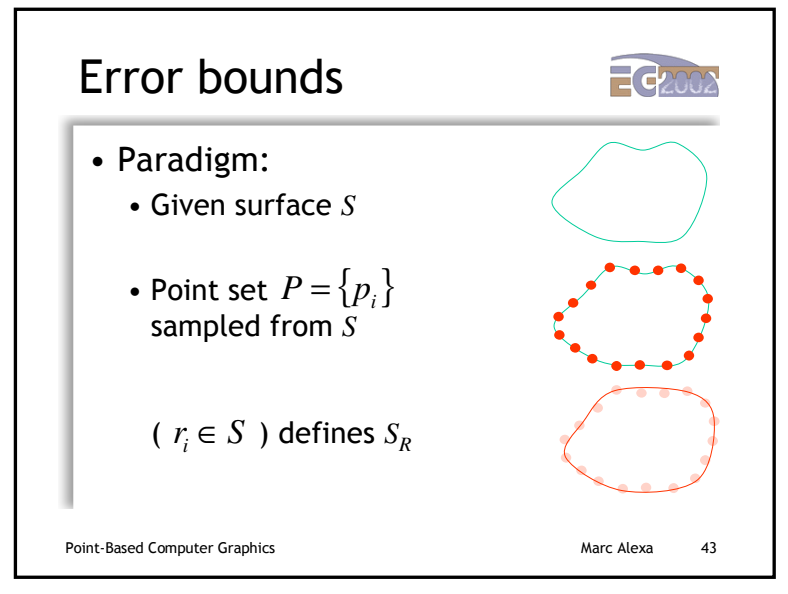

\section{Error bounds}

\section{areor}

- Conclusions

- Remark: Curvature is a useful criterion only for piecewise linear surfaces

- Generally: Higher order derivatives are not accessible

- Quality of representation is mainly dictated by $h$

- Number of points control $h$

- Increase/decrease number of points to adjust the quality of representation

\section{Error bounds}

- Approximation error of $S_{P}$ to $S$

- MLS error approximating a function $\mathrm{f}$ with a polynomial g: $\|f-g\| \leq M \cdot h^{m+1}$

- $M \in O\left(\left\|f^{(m+1)}\right\|\right)$

- $m$ = degree of polynomial

- $S_{P}$ is approximated by a polynomial in each point

- $\left\|S-S_{p}\right\| \leq M \cdot h^{m+1}$

\section{Conclusions}

\section{Carat}

- Projection-based surface definition

- Surface is smooth and manifold

- Surface may be bounded

- Representation error mainly depends on point density

- Adjustable feature size $\mathrm{h}$ allows to smooth out noise

\section{Some References}

Alexa, Behr, Cohen-Or, Fleishman, Levin, Silva. Point Set Surfaces. IEEE

Visualization 2002, pp. 21-28, 2002

Carr, Beatson, Cherrie, Mitchell, Fright, McCallum, Evans. Reconstruction
and Representation of $3 D$ Objects with Radial Basis Functions. SIGGRAPH 2001 Proc., pp. 67-76, 2001

Hoppe, DeRose, Duchamp, McDonald, Stuetzle. Surface Reconstruction from

unorganized points. SIGGRAPH 1992 Proc., pp. 71-78, 1992

Levin. The approximation power of moving least-squares. Math. Comp.

Levin. Mesh-independent surface interpolation. Curves \& Surfaces 2000

Savchenko, Pasko, Okunev, Kunii. Function representation of solids

reconstructed from scattered surface
Graphics Forum, 14(4): $181-188,1995$

Turk, O'Brien. Shape transformation using variational implicit surfaces.

SIGGRAPH 1999 Proc., pp. 335-342, 1999

Turk, O’Brien. Variational implicit surfaces. Technical Report GITGVU 9915

Georgia Institute of Technology, 1999 


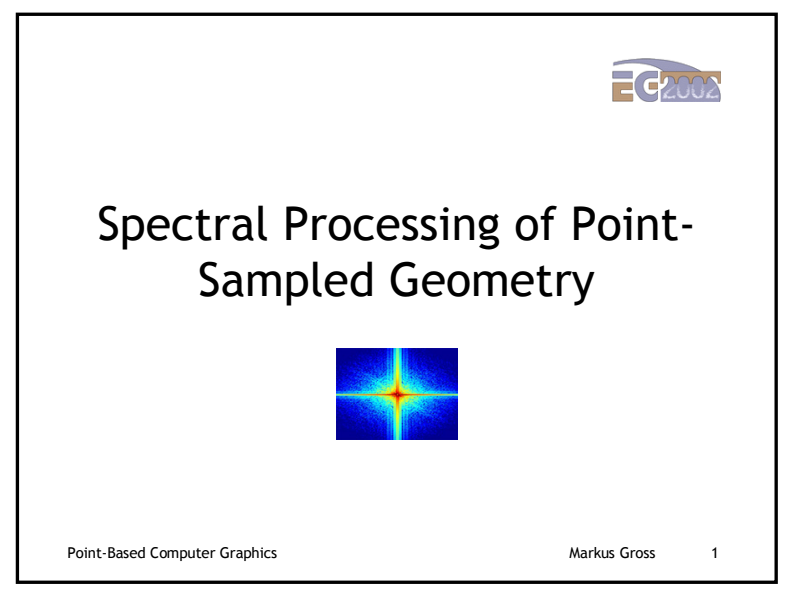

\begin{tabular}{|l|}
\hline Overview \\
\hline - Introduction \\
- Fourier transform \\
- Spectral processing pipeline \\
- Applications \\
- Spectral filtering \\
- Adaptive subsampling \\
- Summary \\
Point-Based computer Graphics
\end{tabular}

\section{Introduction}

- Idea: Extend the Fourier transform to manifold geometry

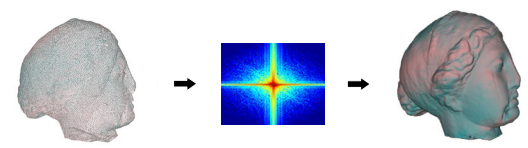

$\Rightarrow$ Spectral representation of point-based objects

$\Rightarrow$ Powerful methods for digital geometry processing

\section{Introduction}

- Applications:

- Spectral filtering:

- Noise removal

- Microstructure analysis

- Enhancement

- Adaptive resampling:

- Complexity reduction

- Continuous LOD

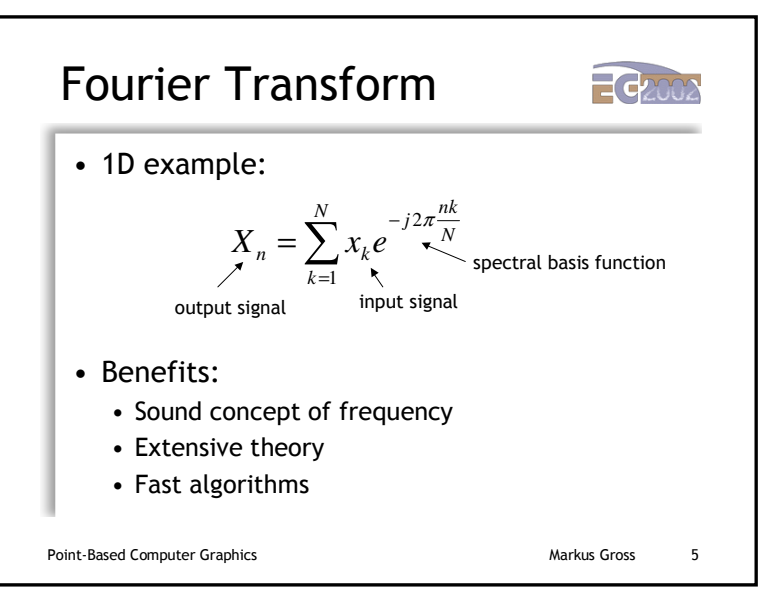

\section{Fourier Transform}

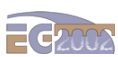

- Requirements:

- Fourier transform defined on Euclidean domain $\Rightarrow$ we need a global parameterization

- Basis functions are eigenfunctions of Laplacian operator

$\Rightarrow$ requires regular sampling pattern so that basis functions can be expressed in analytical form (fast evaluation)

- Limitations:

- Basis functions are globally defined $\Rightarrow$ Lack of local control 

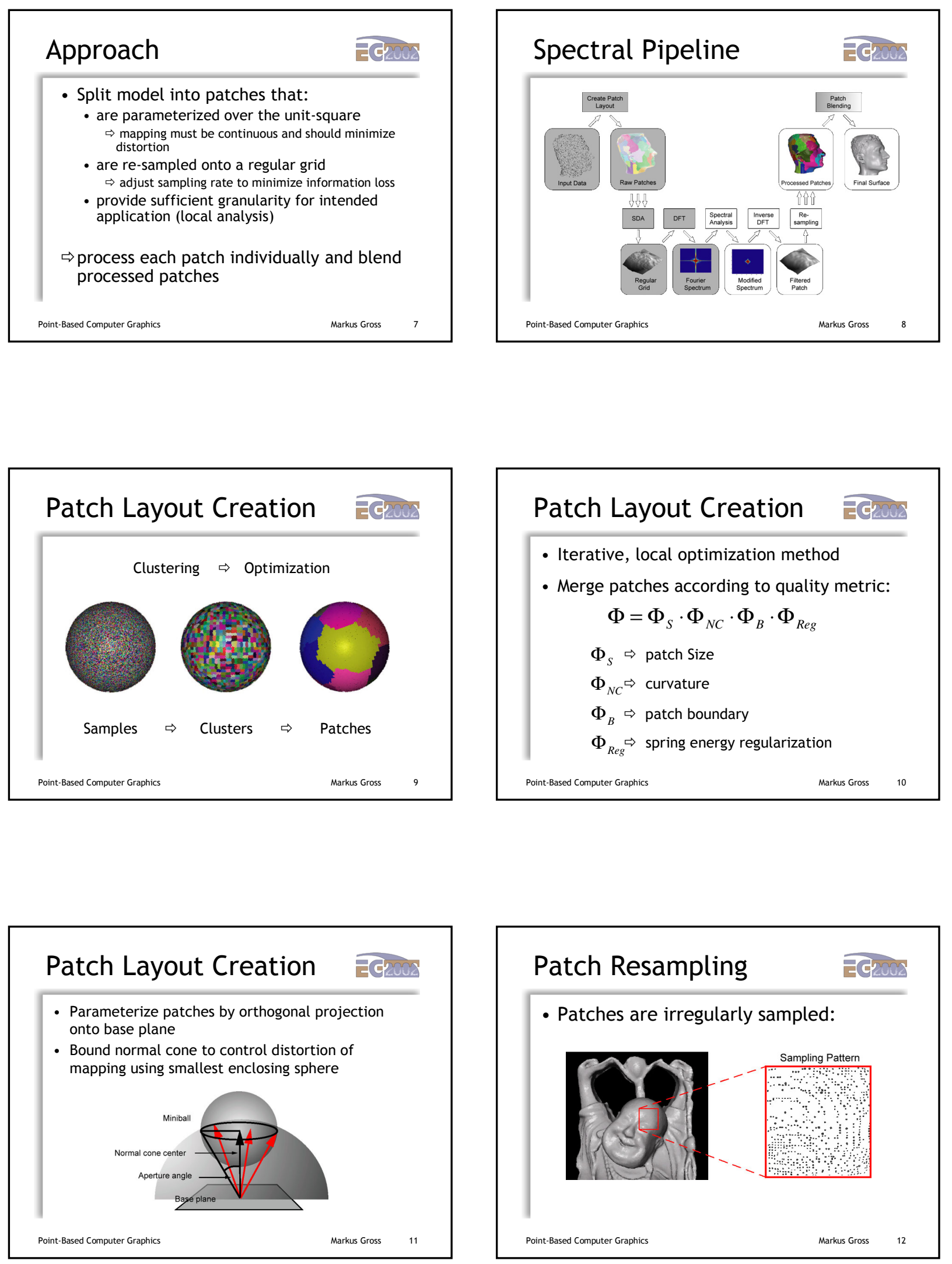


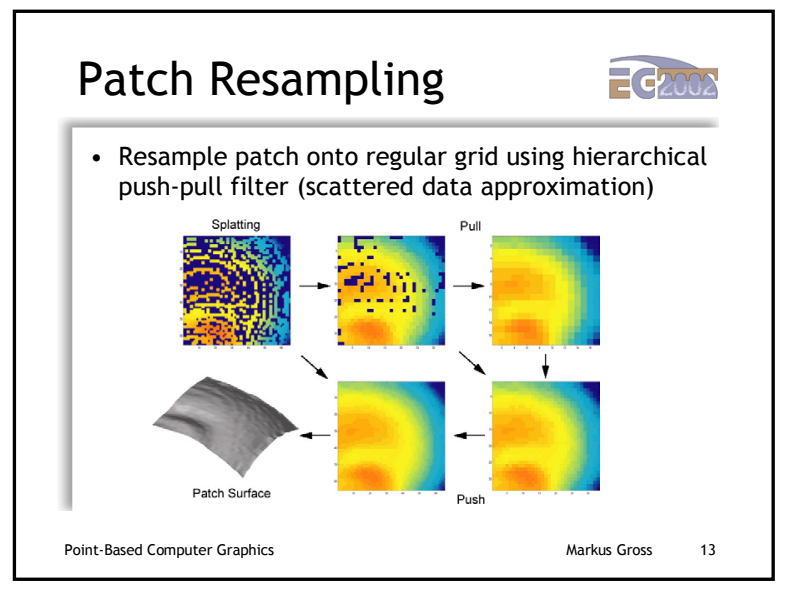

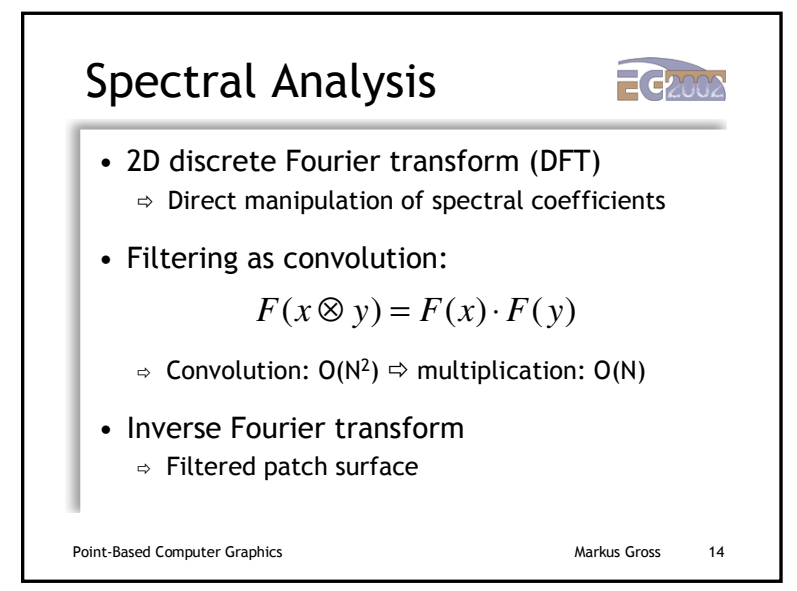
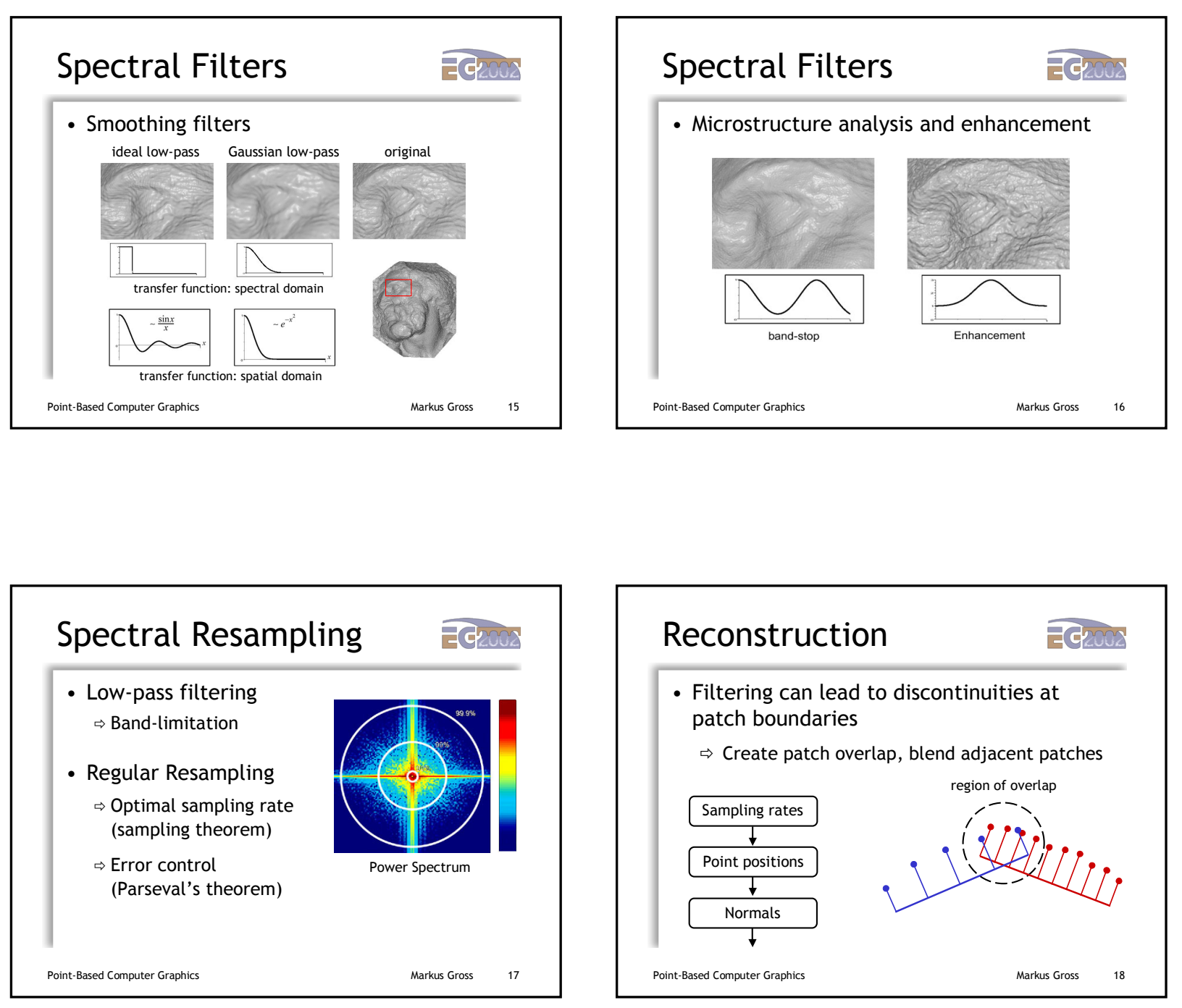

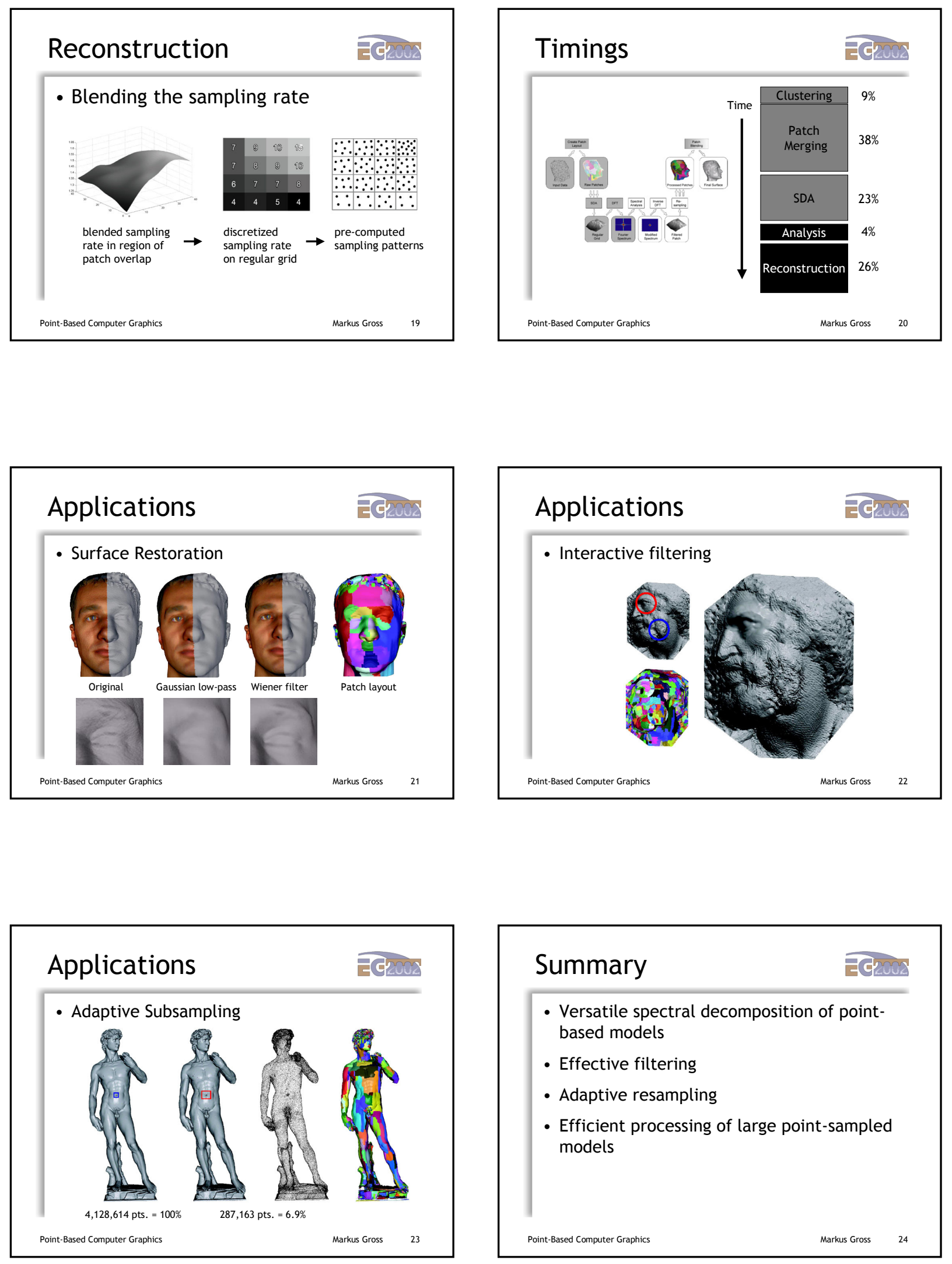

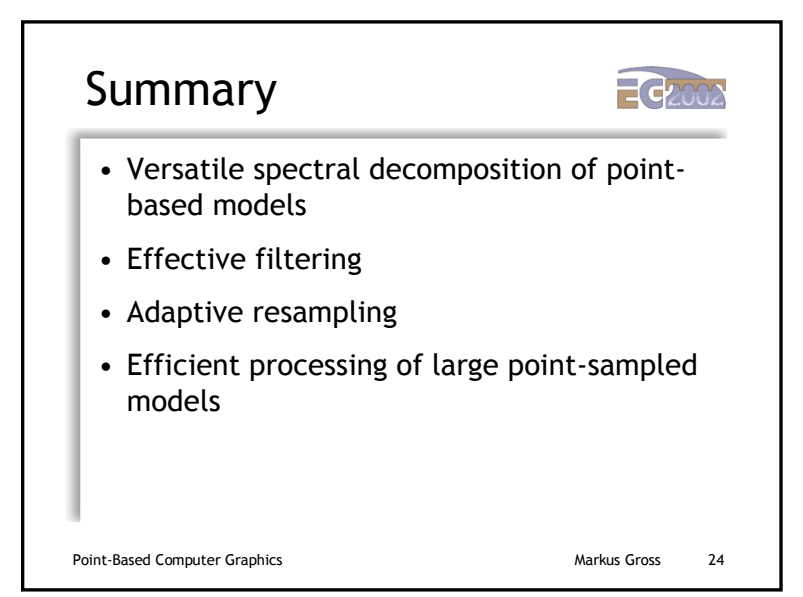




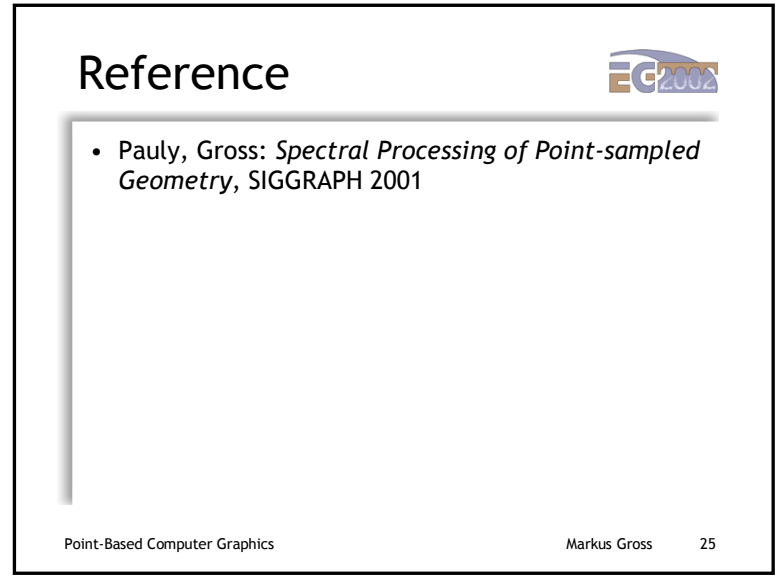




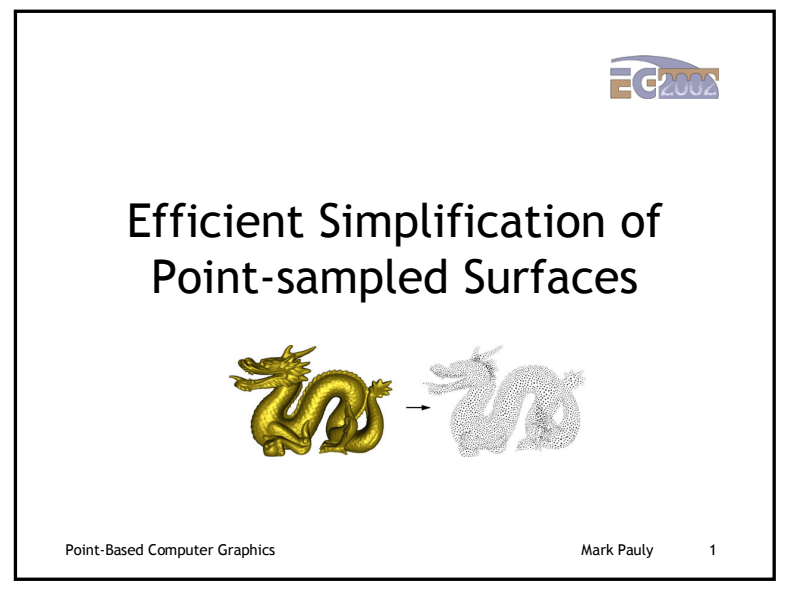

\begin{tabular}{|l|}
\hline Overview \\
\hline - Introduction \\
- Local surface analysis \\
- Simplification methods \\
- Error measurement \\
- Comparison \\
\\
\\
Point-Based computer Graphics \\
\end{tabular}

\section{Introduction}

\section{람over}

- Point-based models are often sampled very densely

- Many applications require coarser approximations,

e.g. for efficient

$$
\begin{aligned}
& \text { - Storage } \\
& \text { - Transmission } \\
& \text { - Processing } \\
& \text { - Rendering }
\end{aligned}
$$

$\Rightarrow$ we need simplification methods for reducing the complexity of point-based surfaces

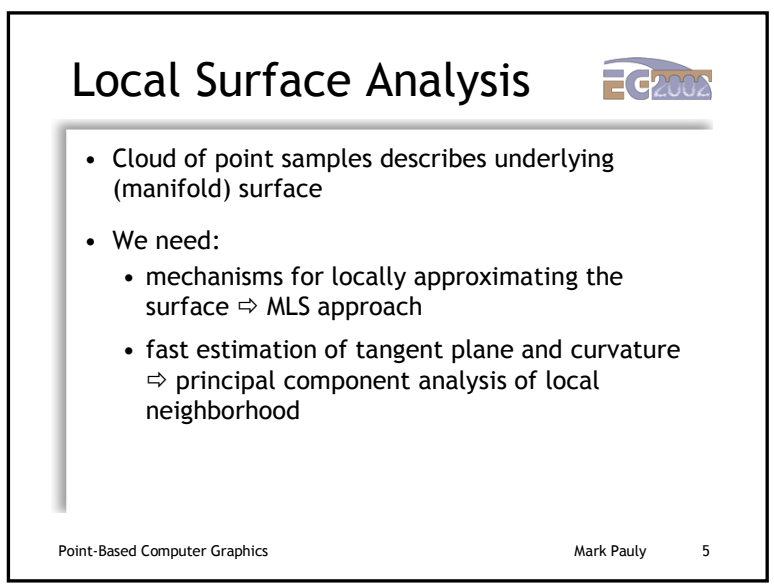

\section{Neighborhood}

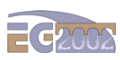

- No explicit connectivity between samples (as with triangle meshes)

- Replace geodesic proximity with spatial proximity (requires sufficiently high sampling density!)

- Compute neighborhood according to Euclidean distance 

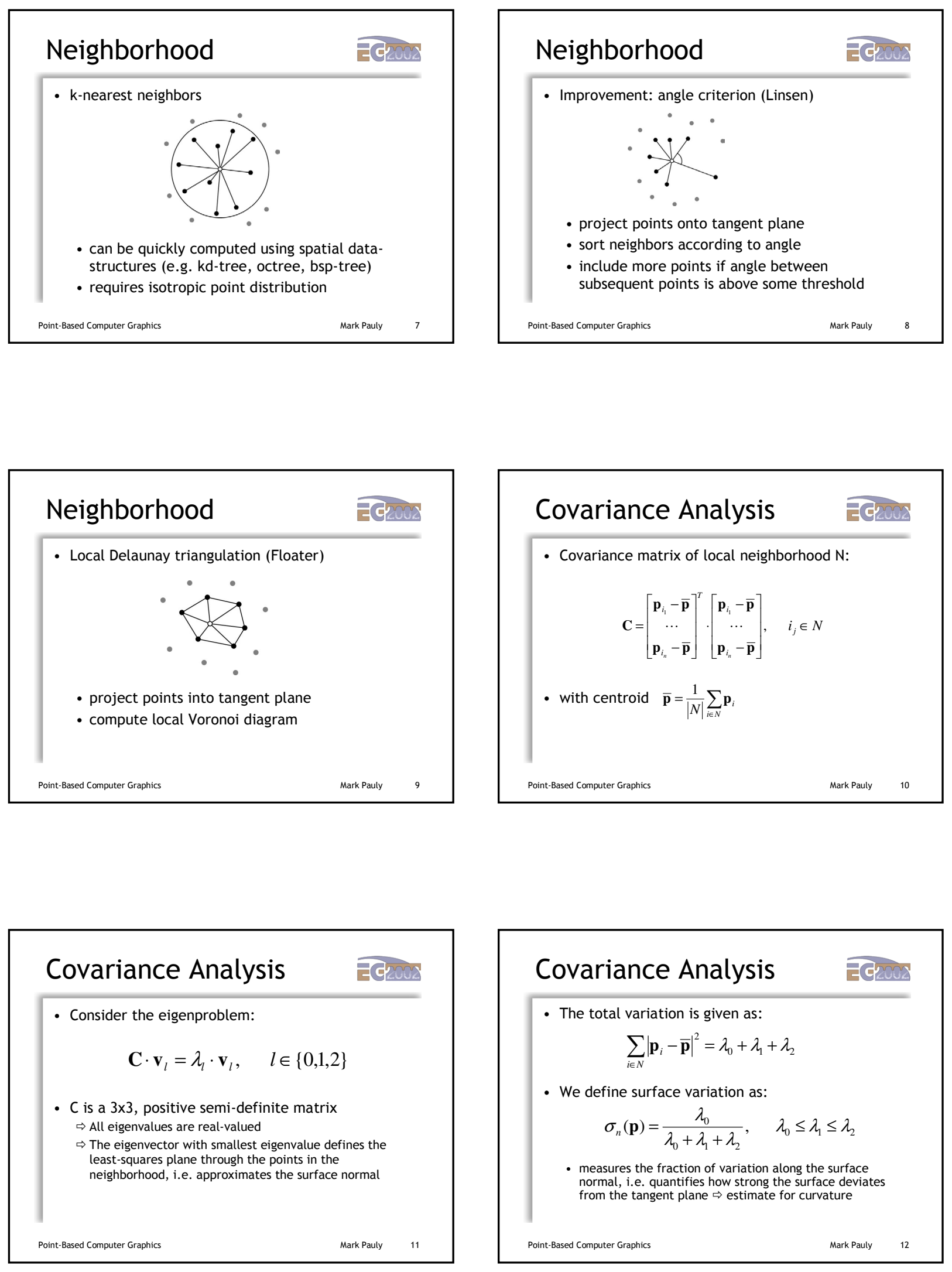

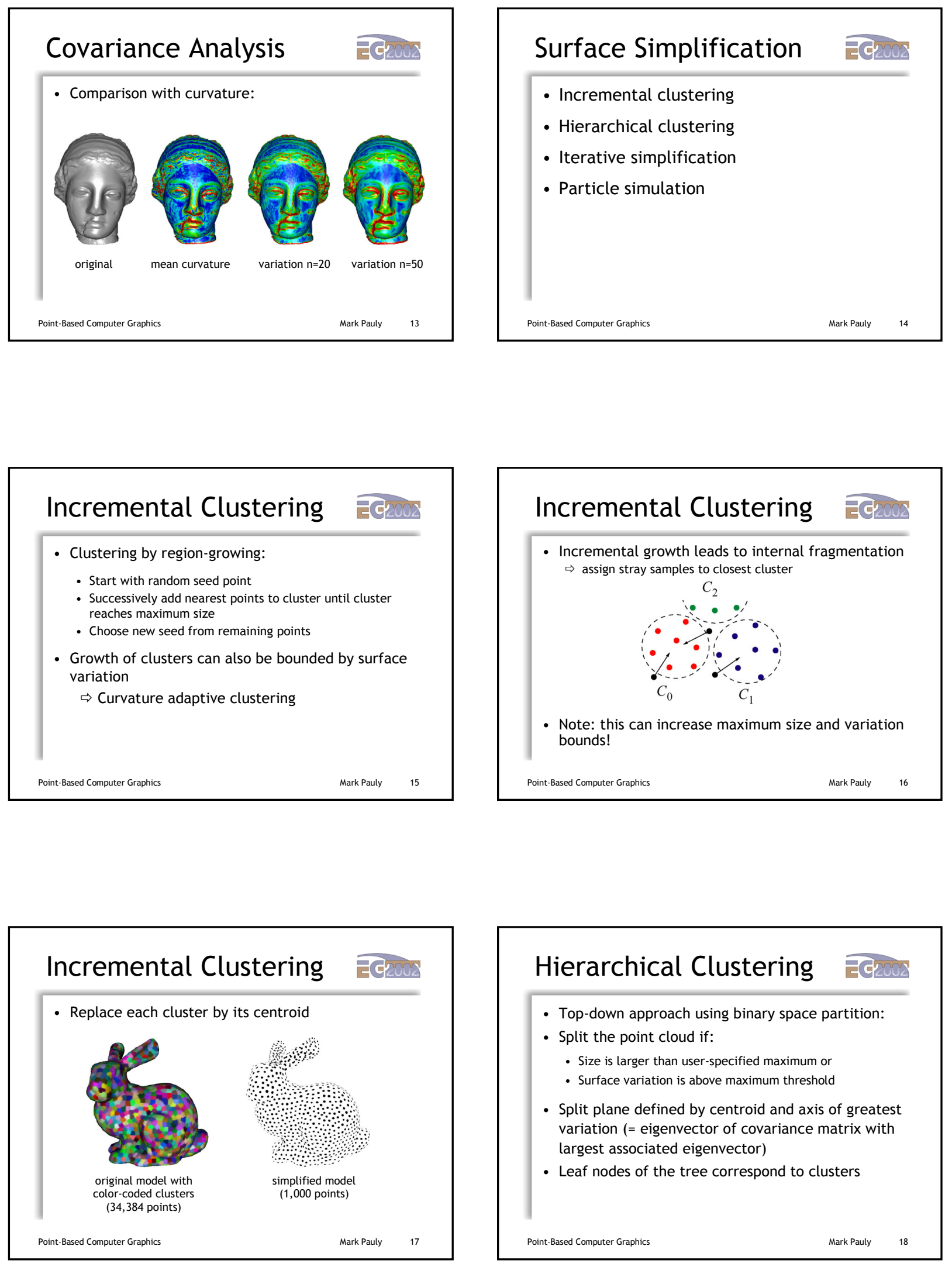

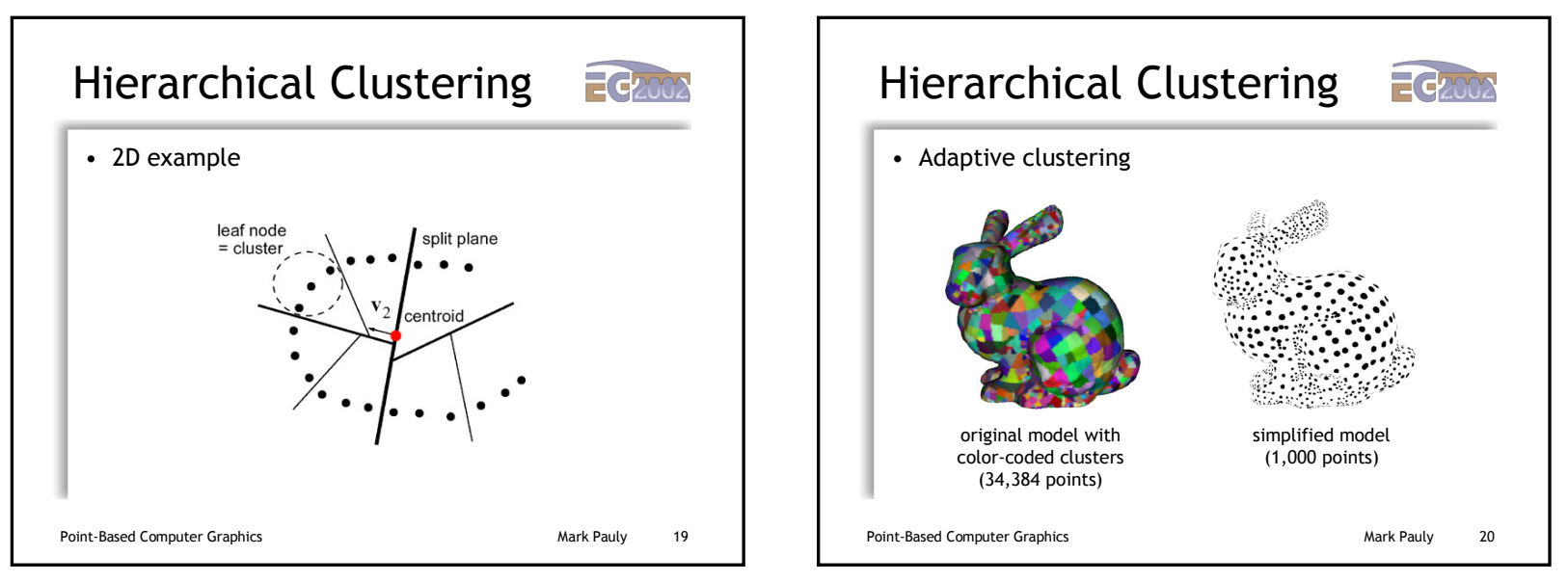

\section{Iterative Simplification 람ove}

- Iteratively contracts point pairs

$\Rightarrow$ Each contraction reduces the number of points by one

- Contractions are arranged in priority queue according to quadric error metric (Garland and Heckbert)

- Quadric measures cost of contraction and determines optimal position for contracted sample

- Equivalent to QSlim except for definition of approximating planes

\section{Iterative Simplification}

- Quadric measures the squared distance to a set of planes defined over edges of neighborhood

- plane spanned by vectors $\mathbf{e}_{1}=\mathbf{p}_{i}-\mathbf{p}$ and $\mathbf{e}_{2}=\mathbf{e}_{1} \times \mathbf{n}$

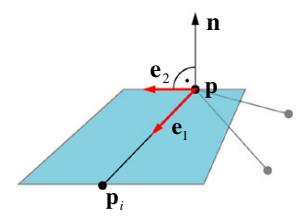

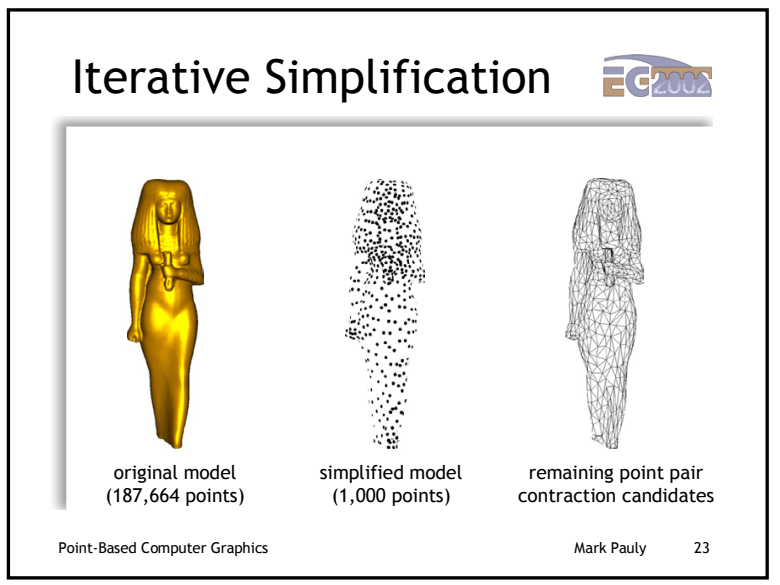

Particle Simulation

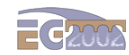

- Resample surface by distributing particles on the surface

- Particles move on surface according to interparticle repelling forces

- Particle relaxation terminates when equilibrium is reached (requires damping)

- Can also be used for up-sampling! 

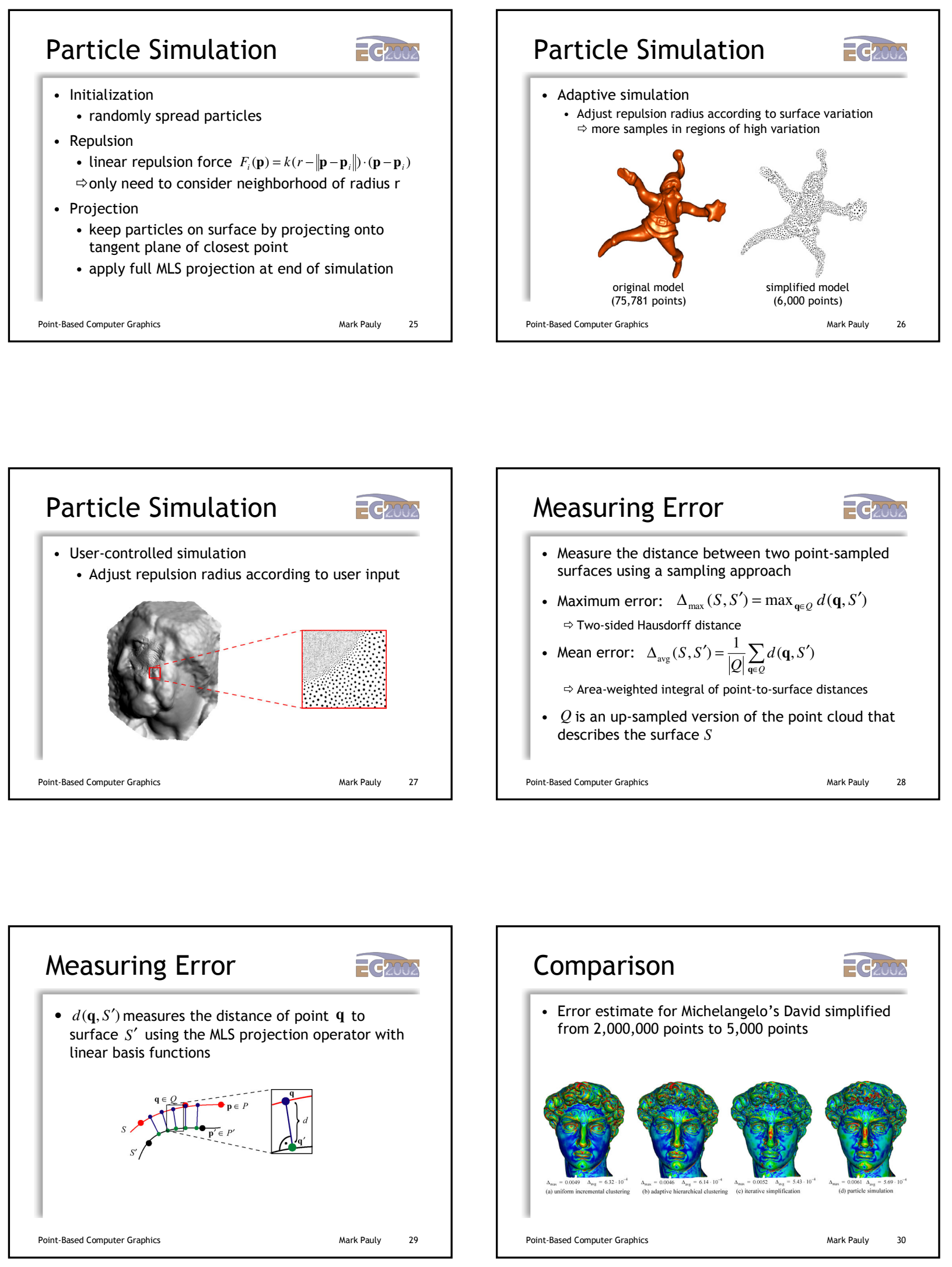

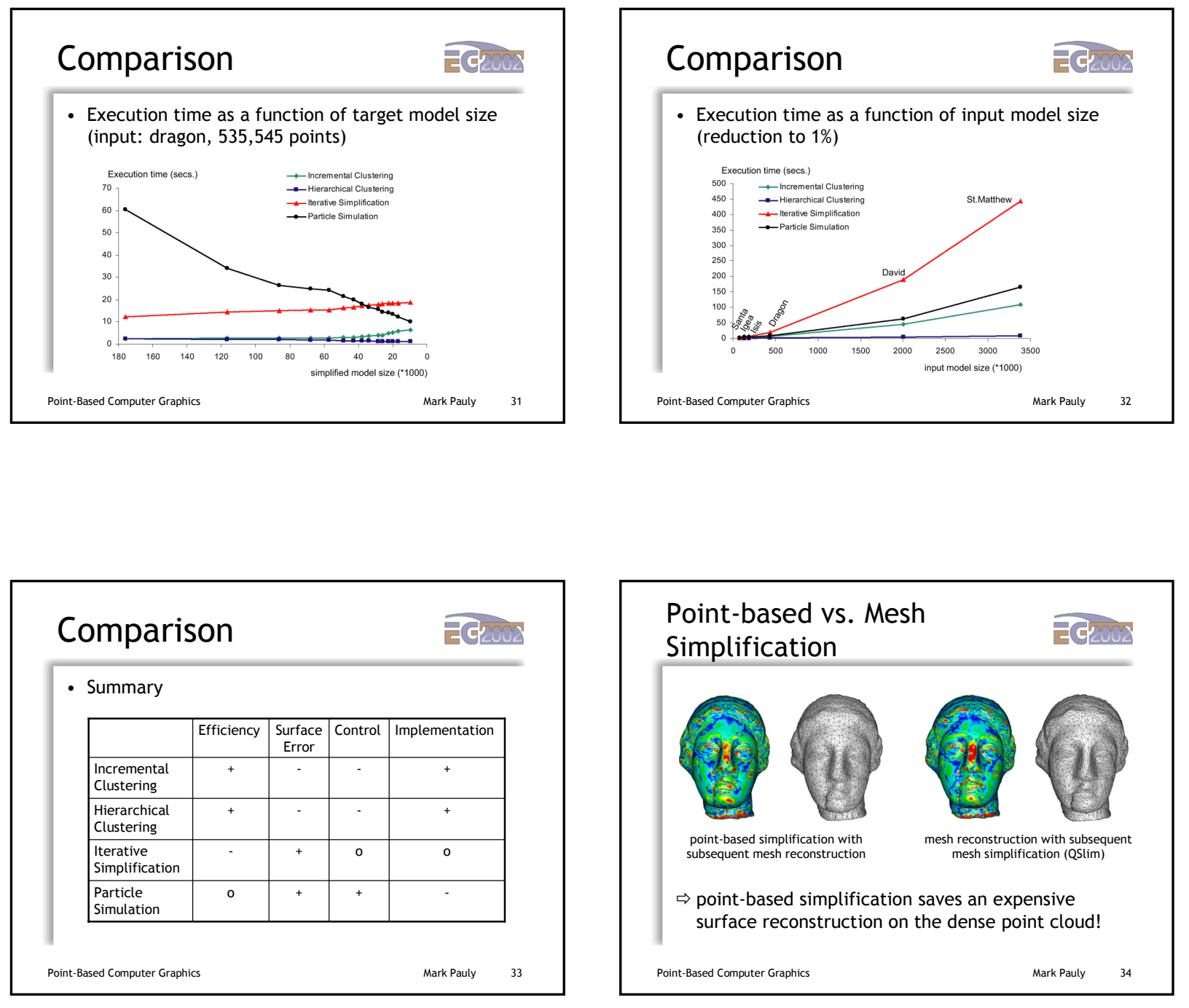

\section{References}

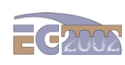

- Pauly, Gross: Efficient Simplification of Pointsampled Surfaces, IEEE Visualization 2002

- Shaffer, Garland: Efficient Adaptive Simplification of Massive Meshes, IEEE Visualization 2001

- Garland, Heckbert: Surface Simplification using Quadric Error Metrics, SIGGRAPH 1997

- Turk: Re-Tiling Polygonal Surfaces, SIGGRAPH 1992

- Alexa et al. Point Set Surfaces, IEEE Visualization 2001 


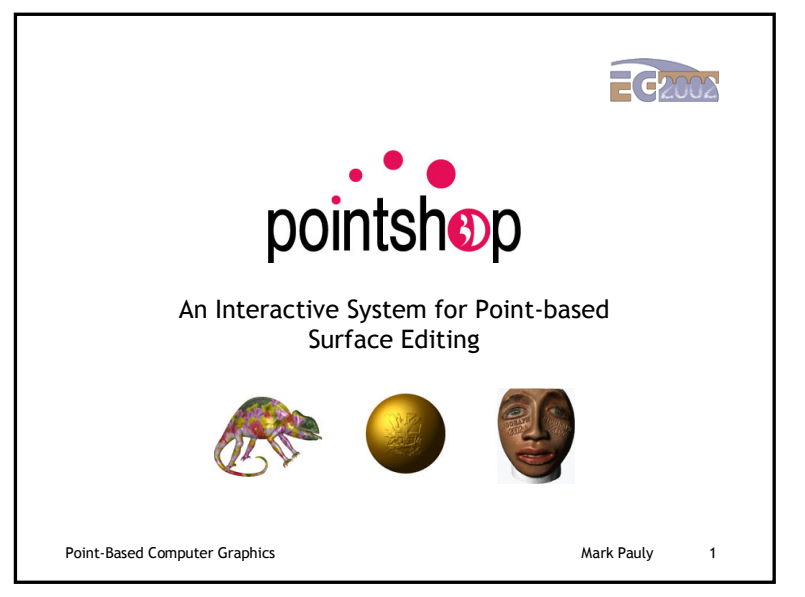

\begin{tabular}{|l|}
\hline Overview \\
\hline - Introduction \\
- Pointshop3D System Components \\
- Point Cloud Parameterization \\
- Resampling Scheme \\
- Editing Operators \\
- Summary \\
\\
Point-Based computer rraphics \\
\hline
\end{tabular}
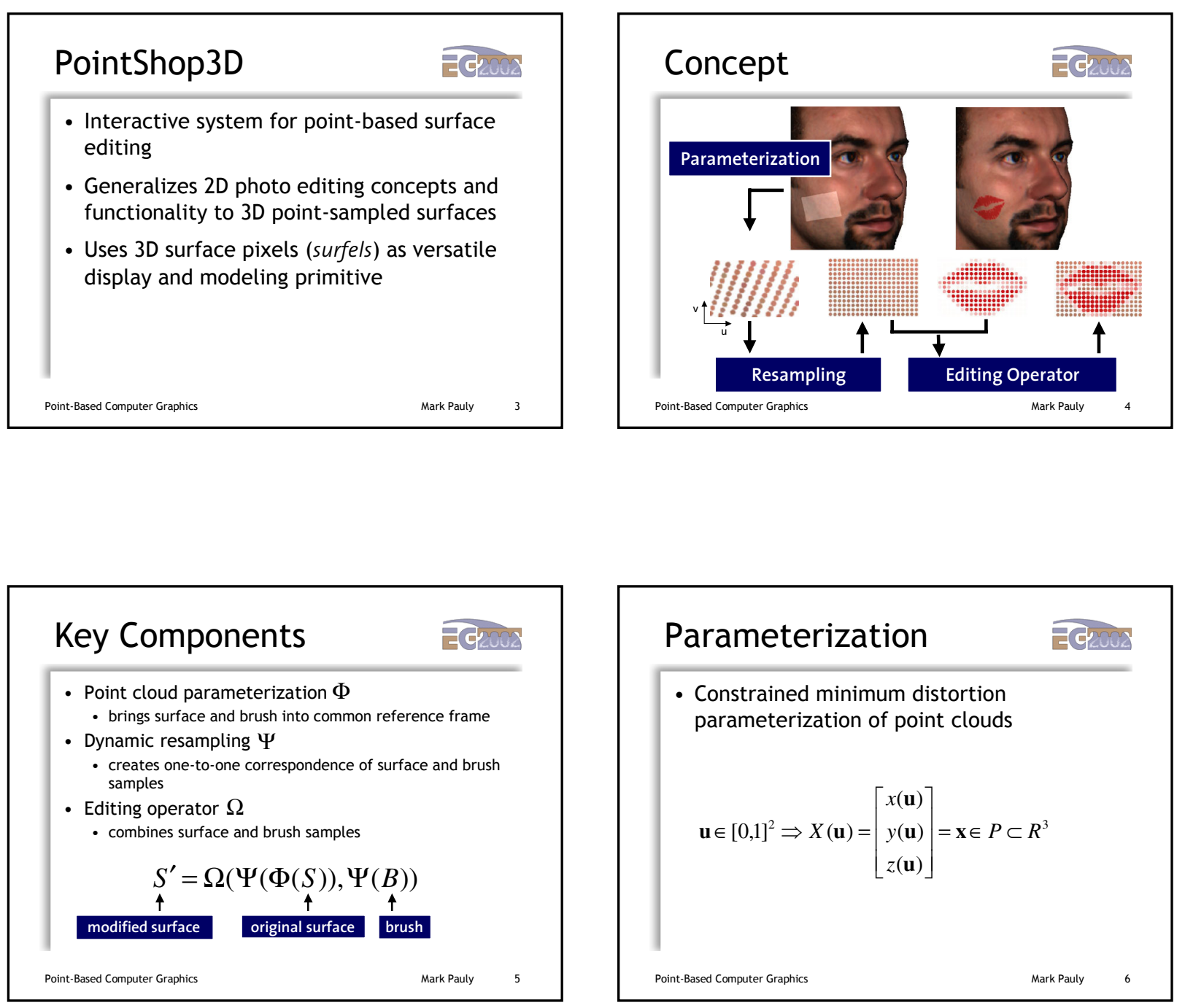

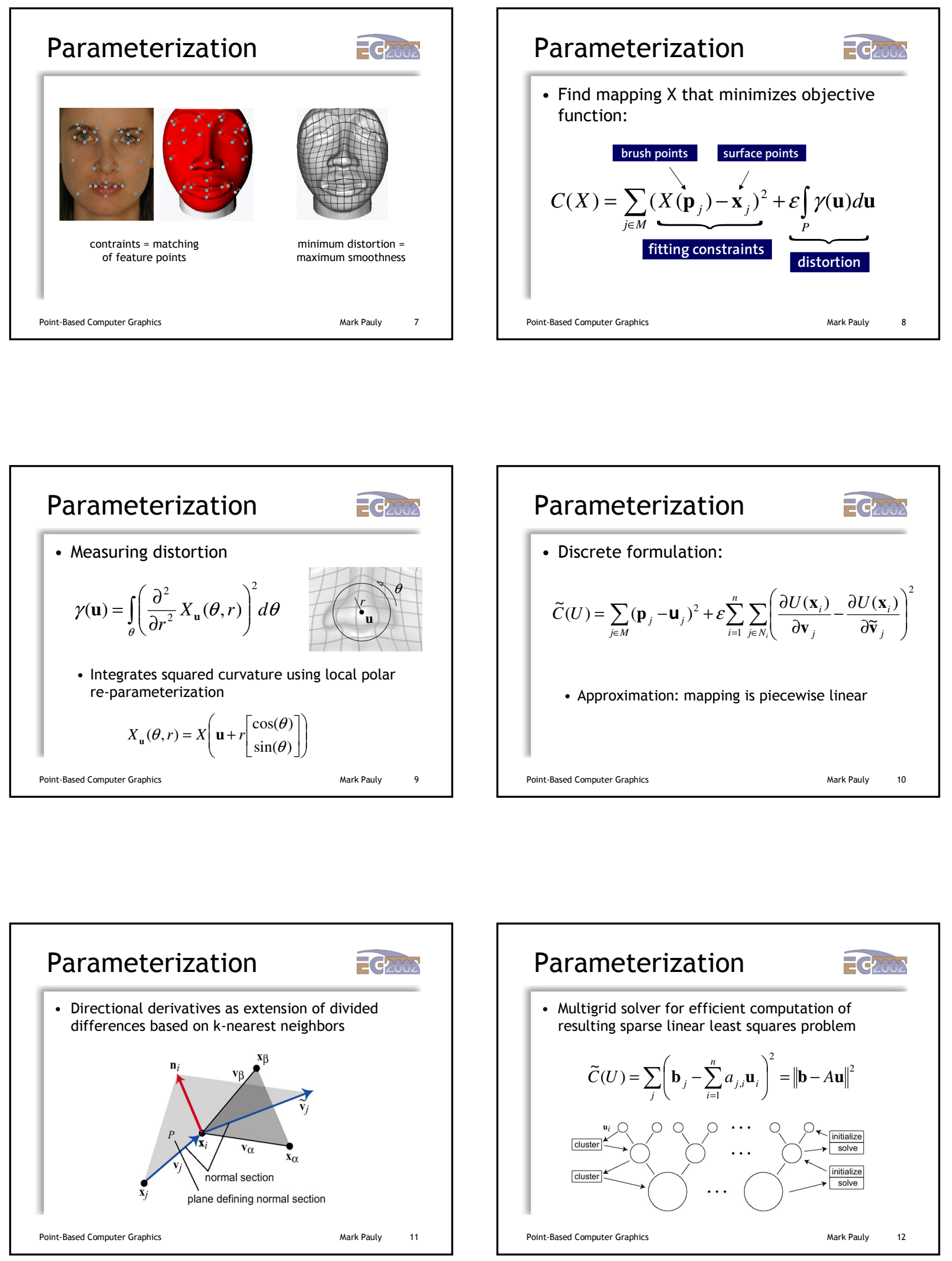

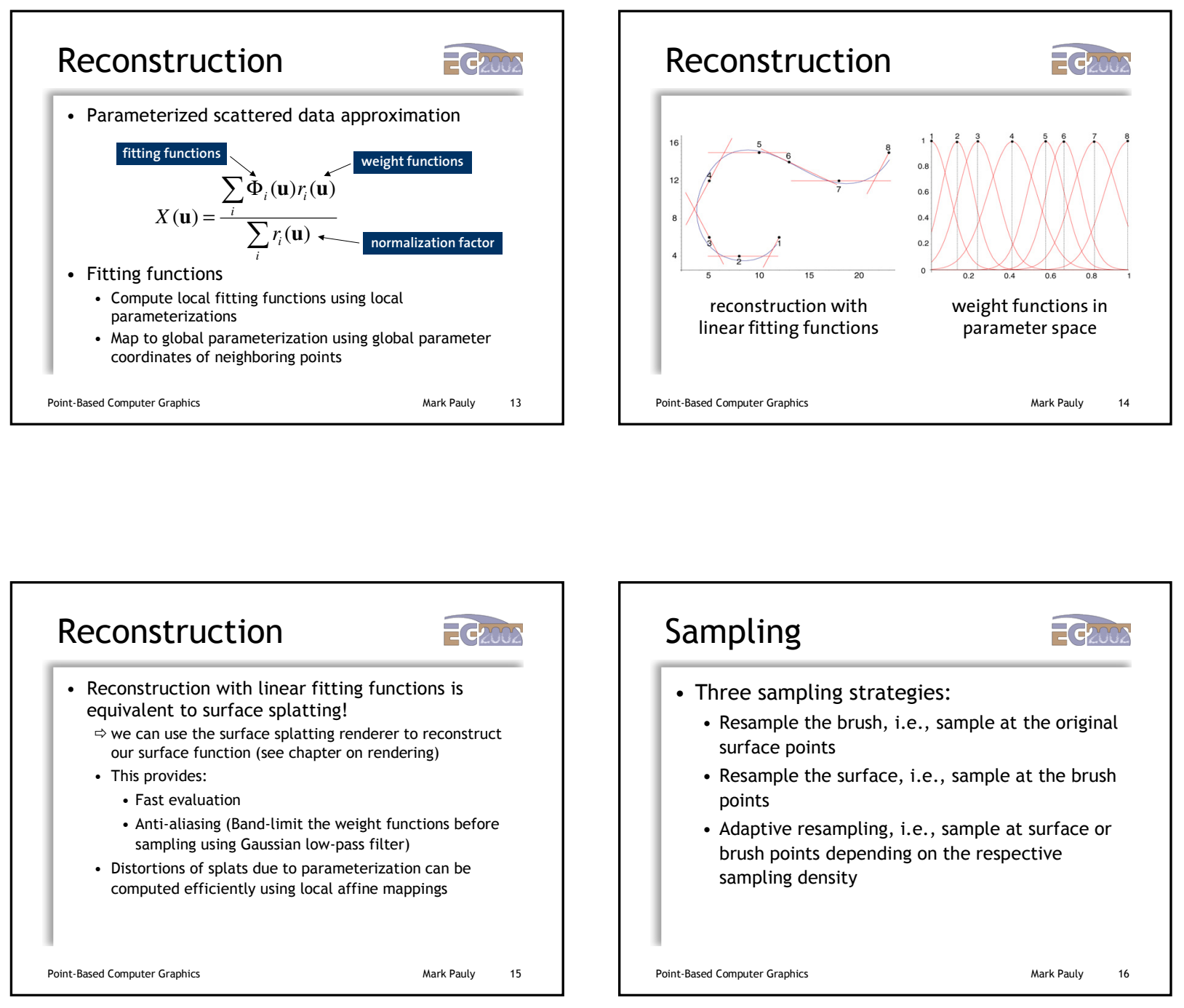

\begin{tabular}{|l|}
\hline Sampling \\
- Three sampling strategies: \\
- Resample the brush, i.e., sample at the original \\
surface points \\
- Resample the surface, i.e., sample at the brush \\
points \\
- Adaptive resampling, i.e., sample at surface or \\
brush points depending on the respective \\
sampling density \\
Point-Based computer Graphics
\end{tabular}
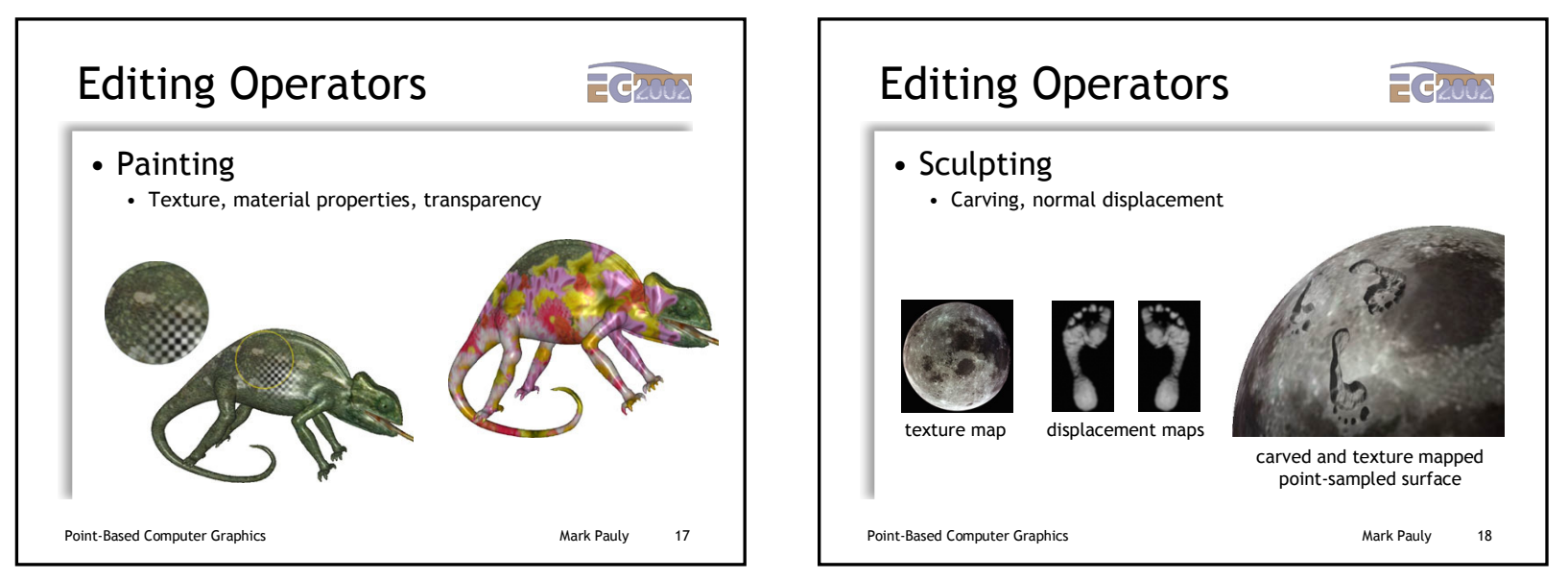


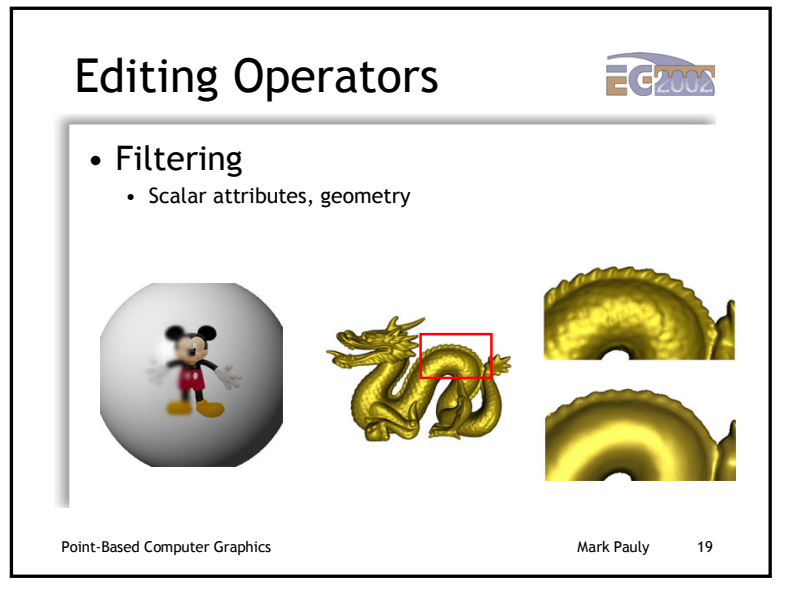

\begin{tabular}{|l|}
\hline Summary \\
\hline - Pointshop3D provides sophisticated editing \\
operations on point-sampled surfaces \\
$\Rightarrow$ points are a versatile and powerful modeling \\
primitive \\
- Limitation: only works on "clean" models \\
- sufficiently high sampling density \\
- no outliers \\
- little noise \\
$\Rightarrow$ requires model cleaning (integrated or as pre- \\
process) \\
Point-Based computer Graphics
\end{tabular}

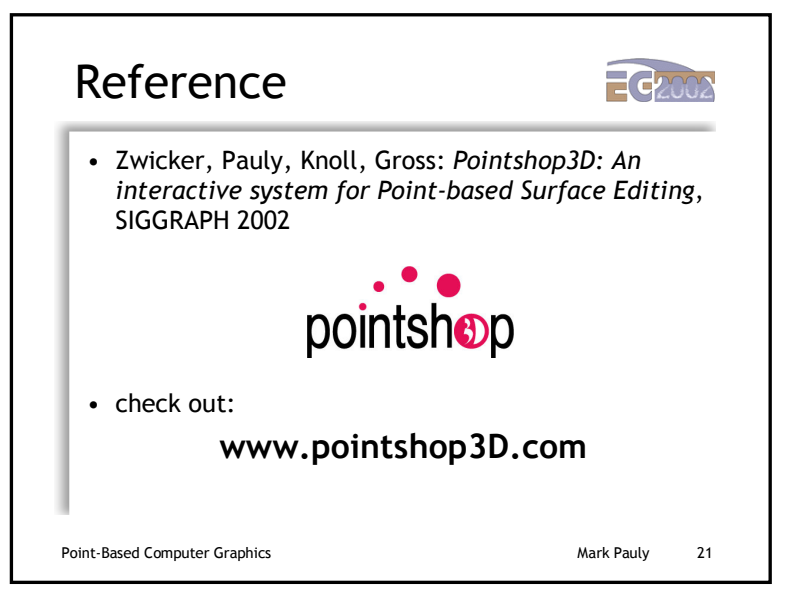

